$224+120+80$ FASTHEACTOA

ANL-7565

ANL-7565

Argonne Mational Laboratorn

WEIGHTED-RESIDUAL METHODS IN SPACE-DEPENDENT REACTOR DYNAMICS

by

Edward L. Fuller III 


\section{DISCLAIMER}

This report was prepared as an account of work sponsored by an agency of the United States Government. Neither the United States Government nor any agency Thereof, nor any of their employees, makes any warranty, express or implied, or assumes any legal liability or responsibility for the accuracy, completeness, or usefulness of any information, apparatus, product, or process disclosed, or represents that its use would not infringe privately owned rights. Reference herein to any specific commercial product, process, or service by trade name, trademark, manufacturer, or otherwise does not necessarily constitute or imply its endorsement, recommendation, or favoring by the United States Government or any agency thereof. The views and opinions of authors expressed herein do not necessarily state or reflect those of the United States Government or any agency thereof. 


\section{DISCLAIMER}

Portions of this document may be illegible in electronic image products. Images are produced from the best available original document. 
The facilities of Argonne National Laboratory are owned by the United States Government. Under the terms of a contract (W-31-109-Eng-38) between the U. S. Atomic Energy Commission, Argonne Universities Association and The University of Chicago, the University employs the staff and operates the Laboratory in accordance with policies and programs formulated, approved and reviewed by the Association.

\section{MEMBERS OF ARGONNE UNIVERSITIES ASSOCIATION}

The University of Arizona Carnagie-Mellnn University

Case Western Reserve University

The University of Chicago

University of Cincinnati

Illinois Institute of Technology

University of Illinois

Indiana University

Iowa State University

The University of Iowa
Kansas State University The University of Kansas Loyola University Marquette University Michigan State University The University of Michigan University of Minnesota University of Missour Northwestern University University of Notre Dame
The Ohio State University Ohio University 'I'he Yennsylvania State Univer sily Furdue University Saint Louis University Southern Illinois University University of Texas Washingtun Unives sity Wayne State University The University of Wisconsin

\section{LEGAL NOTICE}

This report was prepared as an accounl of Guvermment eponsored winrk Neither the United States, nor the Commission, nor any person acting on behalf of the Commission:

A. Makes any warranty or representation, expressed or implied, with respect to the accuracy, completeness, or usefulness of the information contained in this report, or that the use of any information, apparatus, method, or process disclosed in this report may not infringe privately owned rights; or

B. Assumes any liabilities with respect to the use of, or for damages resulting from the use of any information, apparatus, method, or process disclosed in this report.

As used in the above, "person acting on behalf of the Commission" includes any employee or contractor of the Commission, or employee of such contractor, to the extent that such employee or contractor of the Commission, or employee of such contractor prepares, disseminates, or provides access to, any information pursuant to his employment or contract with the Commission, or his employment with such contractor.

Printed in the United States of America

Available from

Clearinghouse for Federal Scientific and Technical Information National Bureau of Standards, U. S. Department of Commerce

Springfield, Virginia 22151

Price: Printed Copy $\$ 3.00$; Microfiche $\$ 0.65$ 


\section{ARGONNE NATIONAL LABORATORY}

9700 South Cass Avenue

Argonne, Illinois 60439

\section{WEIGHTED-RESIDUAL METHODS IN SPACE-DEPENDENT REACTOR DYNAMICS \\ by}

Edward L. Fuller III

Reactor Physics Division

April 1969

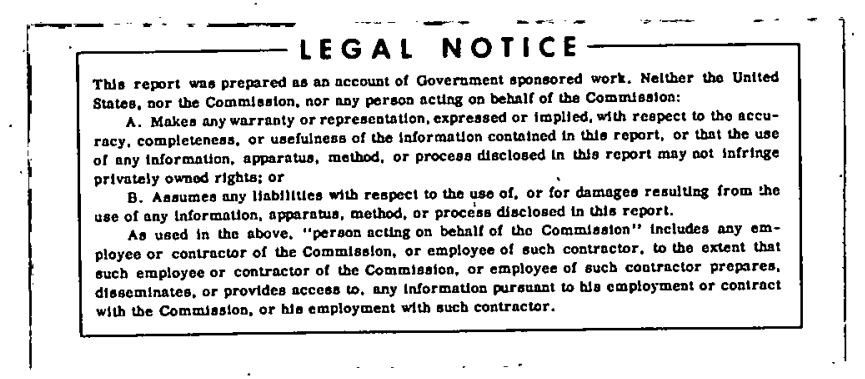

A Dissertation Submitted to the Faculty of the

Department of Nuclear Engineering

in Partial Fulfillment of the Requirements

for the Degree of

Docter of Philosophy

in the Graduate College of

The University of Arizona 


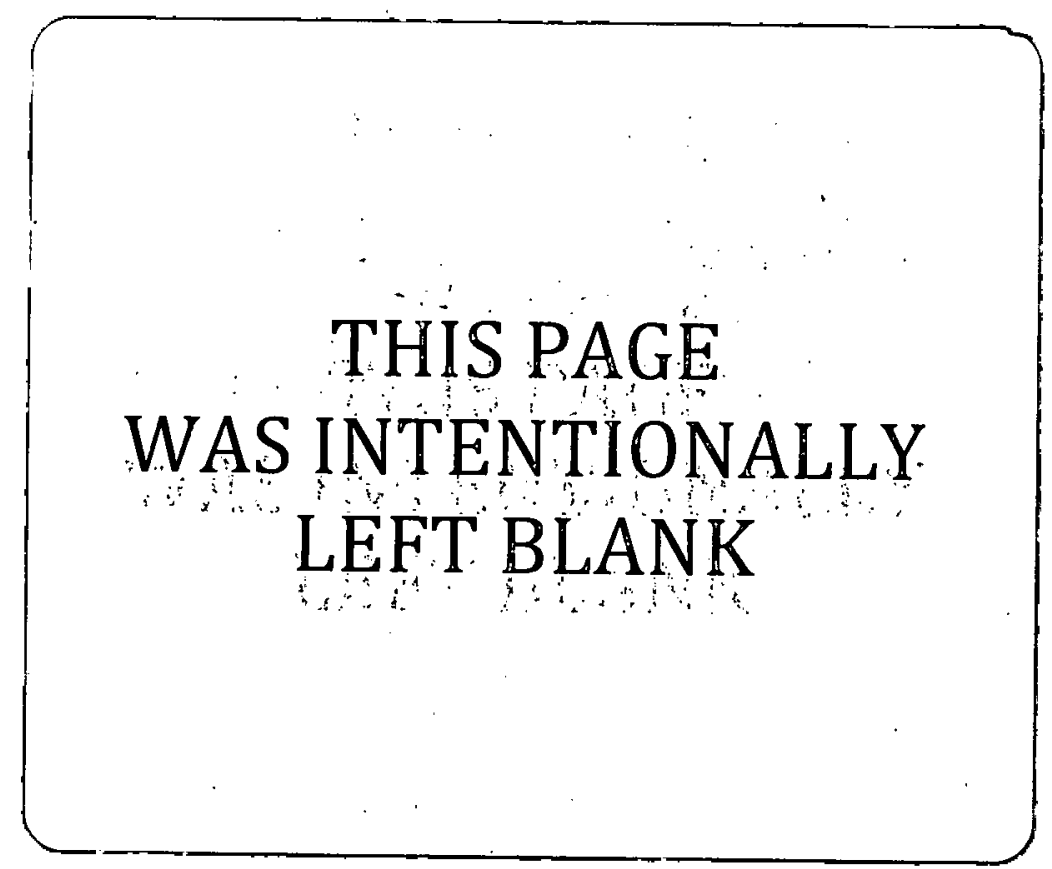


TABLE OF CONTENTS

$\underline{\text { Page }}$

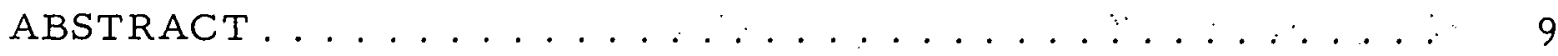

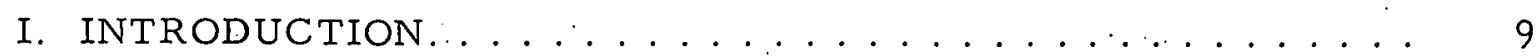

A. Dynamic Problems in Nuclear Reactors . . . . . . . . 9

B. The Time-dependent Multigroup Diffusion Equations . . . . 11

C. Approximate Methods for the Space-Time Problem...... 13

II. THE METHOD OF WEIGHTED RESIDUALS. . . . . . . . . . . . . . 16

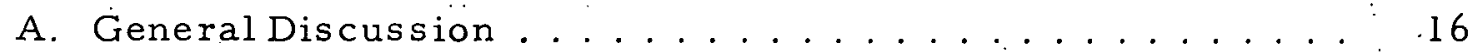

B. Weighted-residual Criteria .............. 18

1. The Method of Collocation .............. 18

2. The Method of Subdomains ............. 18

3. The Method of Least Squares ............. 19

4. The Method of Moments.................. 19

5. The Galerkin Method.................. 20

6. Variational Methods ............... 20

C. Initial Conditions and Boundary Conditions. . . . . . . . 22

D. Some Observations on Accuracy. . . . . . . . . . . 23

E. An Iterative Technique to Improve Accuracy . . . . . . . . 23

III. APPROXIMATE SOLUTION OF THE TIME-DEPENDENT

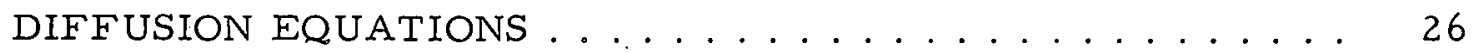

A. The Method of Undetermined Functions . . . . . . . . . 26

B. The Multimode Kinetics Equations . . . . . . . . . . . 29

C. The Point-reactor Model ................... 33

D. Continuous Spatial Modes ................. 34

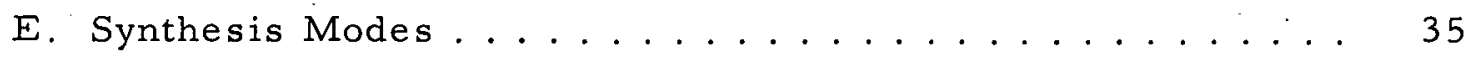

F. The Adjoint Problem: A Variational Principle........ 36 
TABLE OF CONTENTS

Page

IV. SPATIALLY DISCONTINUOUS TRIAL FUNCTIONS . . . . . . 39

A. Spatial Discontinuities.................. . 39

B. The Role of Interface Residuals in Multichannel Synthesis . 40

C. A Nodal Approximation . . . . . . . . . . . . . . . 48

V. TEMPORALLY DISCONTINUOUS TRIAL FUNCTIONS . . . . . 49

A. Introduction. . . . . . . . . . . . . . . 49

B. A Weighted-residual Treatment of Temporal

Discontinuities................... 50

C. The Quasistatic Method .................. 53

1. Main Features. . . . . . . . . . . . . . . 53

2. Shape-function Improvement Using a Nodal Method ... 56

VI. INTEGRATION OF THE MULTIMODE KINETICS EQUATIONS. 61

A. Formulations of Integral Equations . . . . . . . . . 61

B. The Method of Undetermined Parameters . . . . . . . . 63

C. Piecewise Polynomials as Trial Solutions. . . . . . . 65

D. Choices of Weighting Functions. . . . . . . . . . 66

1. Collocation Weighting. ............ 66

2. Subdomain Weighting .............. 68

3. Galerkin Weighting. ................ 69

VII. SPACE-TIME ITERATION USING THE METHOD OF UNDETERMINED FUNCTIONS. . . . . . . . . . . . 71

A. Description of the Method .............. 71

B. A Simple Illustration. . . . . . . . . . . . . . . . . 73

C. Possible Applications of Space-Time Iteration. . . . . . . 77 
TABLE OF CONTENTS

$\underline{\text { Page }}$

VIII. NUMERICAL RESULTS . . . . . . . . . . . . . . . . . . . 79

A. Introduction . . . . . . . . . . . . . . 79

B. Continuous Synthesis . . . . . . . . . 82

C. Multichannel Synthesis ................ 86

1. Nodal Approximations . . . . . . . . . . . . . 86

2. Two Modes in Each Channel ............ 87

IX. CONCLUSIONS AND RECOMMENDATIONS FOR FURTHER

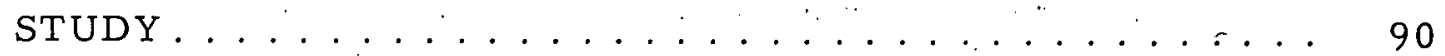

A. Conclusions ........................ 90

B. Recommendations for Further Study.......... 91

APPENDIXES

A. Linear Dependence : . . . . . . . . . . . . . . 92

B. Selection of Time-step Size. . . . . . . . . . . $9: 4$

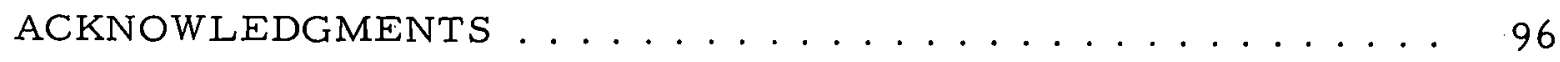

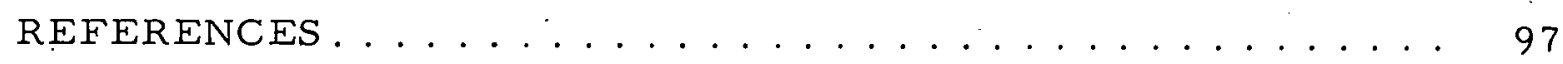




\section{LIST. OF FIGURES}

No.

Title

$\underline{\text { Page }}$

1. A Two-dimensional Reactor Illustrating Spatially

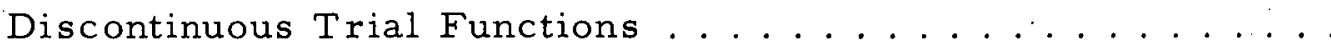

2. Illustration of the Use of Temporally Discontinuous Trial

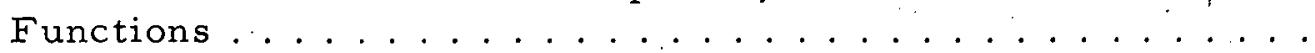

3. Initial State of a One-dimensional Bare Reactor.........

4. Shape Functions Used in Analysis of Transients......... 80

5. Transient 1 Shape Functions at $t=1$ sec, Using "Poor"

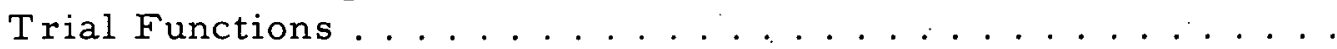

6. Transient 2 Flux Distribution at $t=1$ sec, Using Transient 1 Shapes as Trial Functions ............

7. Transient 2 Shape Functions at $t=1$ sec for 10 Nodes....

8. Transient 2 Shape Functions at $t=1$ sec for Multichannel

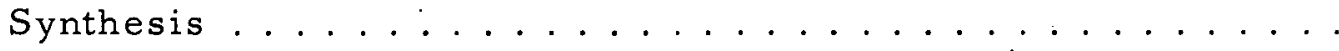




\section{LIST OF TABLES}

No.

Title

$\underline{\text { Page }}$

I. Parameters for Criticality............... 80

II. Region 1. Fission Cross Sections at End of Ramp. . . . . . . 80

III. Transient 1 Results at $t=1$ sec, Using Initial and Final

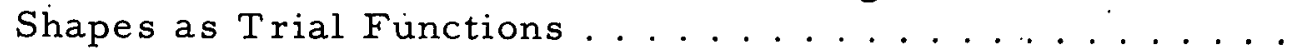

IV. Transient 1 Results at $t=1$ sec, Using "Poor" Trial

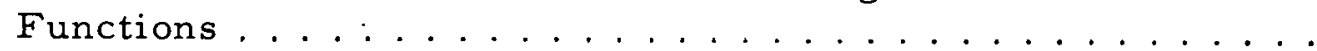

V. Transient 2 Results at $t=1$ sec, Using Initial and Final Shapes as Trial Functions .................

VI. Transient 2 Results at $t=1$ sec, Using Transient 1 Shapes as Trial Functions ................

VII. Interface Locations for Multichannel Approximations . . . . .

VIII. Transient 1 Results at $t=1$ sec for Nodal Approximations.

IX. Transient 2 Results at $t=1$ sec for Nodal Approximations

X. Transient 1 Results at $t=1 \mathrm{sec}$ for Multichannel Synthesis.

XI. Transient 2 Results at $t=1$ sec for Multichannel Synthesis. 


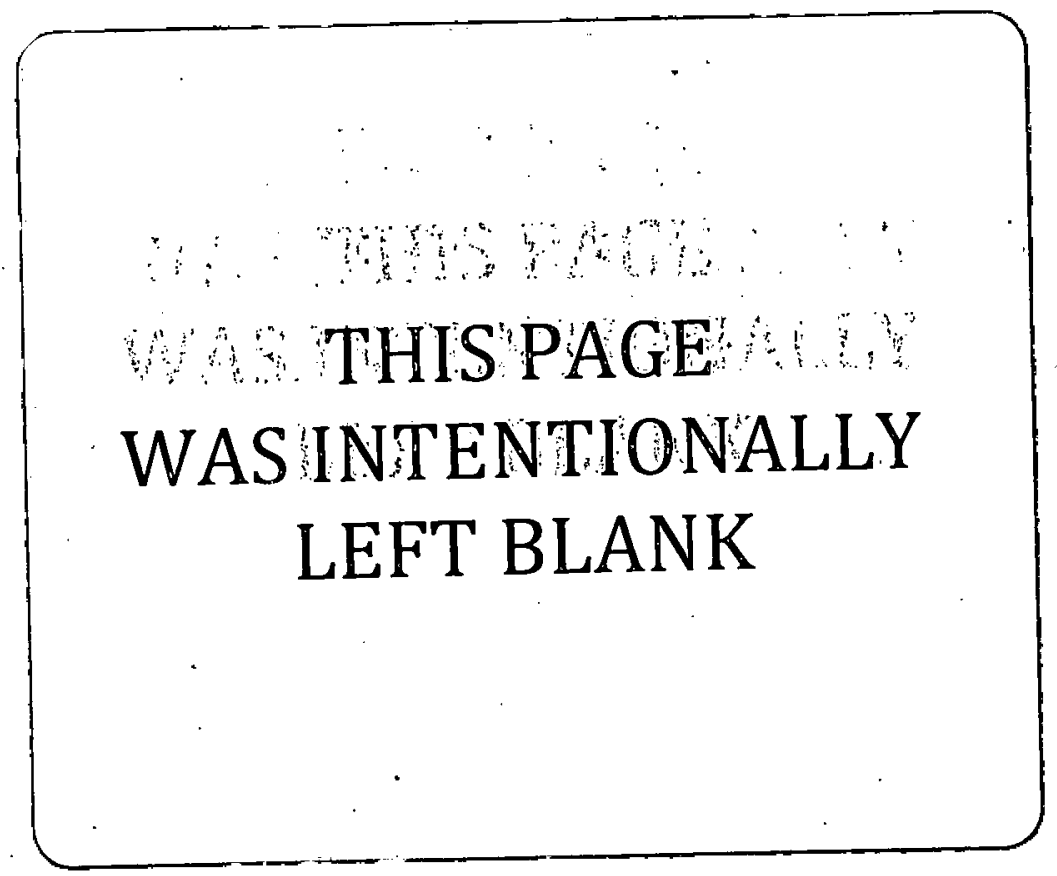




\title{
WEIGHTED-RESIDUAL METHODS IN \\ SPACE-DEPENDENT REACTOR DYNAMICS
}

by

Edwa.rd L. Fuller III

\begin{abstract}
This work is a study of approximate methods for the solution of problems in space-dependent nuclear-reactor dynamics.' It is shown that these approximate methods can all be thought of as applications of the method of weighted residuals. In each method, a trial solution is formed for the neutron flux. The trial solutions are expansions in known spatially dependent functions called trial functions. Each approximate method differs from the others in the manner in which its trial functions are chosen. The undetermined time-dependent functions, called amplitude functions, are then found by using the weighted-residual procedure known as the method of undetermined functions to derive the so-called multimode kinetics equations, which are first-order, ordinary differential equations in time. The multimode kinetics equations are then integrated using the method of undetermined parameters.
\end{abstract}

An iterative method, which uses the method of undetermined functions for both spatial and temporal integrations, is described. It eliminates the need for choosing accurate trial functions because it finds a.solution which is independent of the initial choice of trial functions. A simple example is solved to illustrate the method. Several possible applications of the method are indicated.

Some numerical results are reported for continuous-synthesis and multichannel-synthesis approximations. Several choices of weighting functions are compared. Conclusions are drawn regarding the roles of the trial functions and the.weighting functions in obtaining accurate solutions.

\section{CHAPTER I}

\section{INTRODUCTIÓN}

A. Dynamic Problems in Nuclear Reactors

In the analysis and design of large nuclear power reactors, knowledge of the spatial and temporal behavior of the neutron flux is essential. Localized changes in material compositions; such as control-rod withdrawals, 
cause changes in the neutron-flux shape which contribute to changes in the power level. For this reason, the traditional point-reactor model does not adequately describe the transient behavior of either large fast or thermal reactors.

At least four areas of transient analysis can be distinguished. They are reactor safety studies, analysis for design and routine operation, analysis of xenon oscillations in thermal reactors, and fuel-cycles studies. Each problem area differs from the others in that each has widely different characteristic transient times, which range from milliseconds in severe accident-induced transients to months in a fuel-management program.

Safety studies are directed toward investigating transients initiated by changes in material composition. Knowledge of the response of a reactor to a wide variety of changes can improve the safety of the reactor. Since accidents cannot be predicted beforehand, the prevailing philosophy of reactor safety is to imagine possible severe accidents, analyze them, and design to prevent them as far as possible. Some effects of serious accidents are fuel-element rupture, loss of coolant flow, and core meltdown. If the space-time behavior of the neutron flux is calculated accurately when. analyzing possible accidents, the effects mentioned above could be prevented by designing the reactor properly: Accident analysis is emphasized in the numerical examples of Chapter VIII.

An operating power reactor must routinely satisfy demands for power increases. The power level is adjusted by moving control rods within the reactor. The control-rod movements, which take place over a period of minutes, cause the flux shape to change as the power level changes. The relationships between a control-rod movement and the corresponding flux shape and power-level changes must be properly accounted for in the reactor design.

Xenon oscillations in thermal reactors generally occur with characteristic periods of hours or days. A flux increase in a localized region of a large thermal reactor causes the concentration of the poison ${ }^{135} \mathrm{Xe}$ to increase in that region. As the xenon concentration builds up, the neutron flux decreases in the region relative to the flux in adjacent regions. Since the total power demanded from the reactor remains the same, the flux in the adjacent regions increases still further to meet the demand. The xenon concentration in these regions now increases while decreasing in the original region of increase. The xenon concentration thus has a characteristic period of oscillation. Since the oscillation could be unstable, it should be controlled.

Fiel burnup is usually allowed to take place for many days before replacement or rearrangement. In planning an optimum fuel management program, one should know the spatial effects of fuel burnup. 
Each of the four classes of problems described above can be solved using the space-energy-time-dependent neutron transport equation and the balance equations for the delayed-neutron precursors. In practice, though, one of the approximations to the transport equation is used, such as multigroup diffusion theory. In addition, each type of space-time problem has its own characteristic "feedback" effects. An accident could be initiated by sodium voiding in a fast reactor, and then limited by Doppler feedback, which is a nonlinear phenomenon. A xenon oscillation problem uses a balance equation for the xenon concentration. Isotope balance equations are needed in fuel-burnup studies.

The time scales for each problem type are so vastly different that they can be considered independently from one another. The methods that will be subsequently discussed are applicable to each type of problem, although the focus on detail differs from one application to the next. For example, delayed neutrons are neglected in a fuel-cycles study. Conversely, changes in isotope concentrations are neglected in safety studies.

B. The Time-dependent Multigroup Diffusion Equations

In Section $A$ above, it was noted that the time-dependent multigroup diffusion-theory approximation is often used to solve the space-time problem. In this approximation, the energy variable is discretized into $G$ distinct energy groups, and the resulting system of equations is then written in the compact form ${ }^{1}$

$$
\begin{aligned}
& V^{-1} \frac{\partial \phi}{\partial t}(\vec{r}, t)=\left[\nabla \cdot D \nabla-A+(1-\beta) \chi_{p} F^{T}\right] \phi(\vec{r}, t)+\sum_{j=1}^{J} \lambda_{j} \chi_{j} \eta_{j}(\vec{r}, t) \\
& \frac{\partial \eta_{j}}{\partial t}(\vec{r}, t)=\beta_{j} F^{T} \phi(\vec{r}, t)-\lambda_{j} \eta_{j}(\vec{r}, t) .
\end{aligned}
$$

In Eqs. 1.1 and 1.2, the group fluxes are represented by the column vector

$$
\phi(\vec{r}, t)=\left[\begin{array}{c}
\phi_{1}(\vec{r}, t) \\
\cdot \\
\cdot \\
\phi_{G} \dot{(\vec{r}, t)}
\end{array}\right],
$$

the precursor concentrations by the functions $\eta_{j}(\vec{r}, t), j=1, \ldots ; \mathrm{J}$, and the neutron speeds by the diagonal matrix 


$$
\mathrm{V}^{-1}=\left[\begin{array}{c}
\frac{1}{\mathrm{v}_{1}} \\
\vdots \\
\frac{1}{\mathrm{v}_{\mathrm{G}}}
\end{array}\right]
$$

The macroscopic diffusion coefficients are represented by the diagonal matrix

$$
\mathrm{D}=\left[\begin{array}{cc}
\mathrm{D}_{1} & \\
\cdot & \\
& \cdot \\
& \cdot \\
& \mathrm{D}_{\mathrm{G}}
\end{array}\right]
$$

the macroscopic absorption and scattering cross sections appear in the $G \times G$ matrix

$$
A=\left[\begin{array}{cccc}
\Sigma_{1} & \\
\cdot & \\
\cdot & \\
\cdot & \Sigma_{\mathrm{G}}
\end{array}\right]-\left[\begin{array}{ccc}
\Sigma_{11} & \cdots & \Sigma_{1 \mathrm{G}} \\
\cdot & \cdots & \ddots \\
\cdot & \cdots & \cdot \\
\Sigma_{\mathrm{G}} & \cdots & \Sigma_{\mathrm{GG}}
\end{array}\right] .
$$

The fission cross sections appear in the column matrix

$$
F=\left[\begin{array}{c}
\nu \Sigma_{f 1} \\
\cdot \\
\cdot \\
\nu \dot{\Sigma}_{f G}
\end{array}\right]
$$

$F^{T}$ is the trianspose of $F$ and is thus a row matrix.

To a reasonable approximation, the emission spectrum of prompt neutrons is represented by the column matrix

$$
\chi_{\mathrm{p}}=\left[\begin{array}{c}
\chi_{\mathrm{pl}} \\
\cdot \\
\cdot \\
\chi_{\mathrm{pG}}
\end{array}\right]
$$


the emission spectra of the delayed neutrons are given by

$$
\chi_{\mathbf{j}}=\left[\begin{array}{c}
\chi_{\mathrm{j} 1} \\
\cdot \\
\cdot \\
\chi_{\mathrm{jG}}
\end{array}\right], \quad \mathrm{j}=1, \ldots, \mathrm{J}
$$

The precursor decay constants and the delayed-neutron fractions are $\lambda_{j}$ and $\beta_{j}, j=1, \ldots, J$, respectively; the total delayed-neutron fraction is

$$
\beta=\sum_{j=1}^{J} \beta_{j} .
$$

The system of equations represented by Eqs. 1.1 and 1.2 is generally far too complicated to solve without resorting to numerical and/or approximation techniques. In fact, even a direct numerical solution on a high-speed computer is impractical if more than one space dimension and/or more than a few energy groups are used. The only hope in obtaining solutions to the full space-energy-time-dependent problem seems to lie in the development of good approximation techniques. Some of these attempts will be described next.

C. Approximate Methods for the Space-Time Problem

The numerical methods, such as finite-difference methods, ${ }^{2}$ have traditionally been poor choices for reactor dynamics. The $\mathrm{V}^{-1}$ matrix, which multiplies the time derivative of the flux column vector, contains elements that are very small. Because of this, very small time steps must be taken to ensure a stable solution. Computation becomes very time. consuming, even för relatively sïmple cases. ${ }^{3}$ Consequently, more economical approximate methods have been developed, which have varying degrees of accuracy.

The improved temporal integration techniques can be divided into two classes: those that minimize the finite-difference time-step limitation, and those that overcome it. Examples of the former are the so-called Theta-difference method of Henry and Vota" and the "Streak" method of Smiley. ${ }^{5}$ Examples of methods that overcome the time-step limitation are the exponential matrix approximations of Porsching, ${ }^{6}$ Hansen's largesteigenvalue method, ${ }^{7,8}$ Adler's method, ${ }^{9}$ and the methods belonging to the larger class known as the method of undetermined parameters, such as that developed by Brittan ${ }^{10}$ and improved upon by Kaganove. ${ }^{11}$ The method of undetermined parameters, being a weighted-residual method, is explored thoroughly in this report. One essential feature common to all the methods 
that overcome the time-step limitation is that the equations are formally integrated with respect to the time variable: ${ }^{12}$ Then, slowly varying terms of the integrands are approximated by trial solutions. The methods that overcome the finite-difference time-step limitation are discussed further in Chapter VI.

In addition to the development of improved techniques for carrying out the temporal integrations, approximations for treating the spatial variation of the flux have been propounded. These include the traditional pointreactor model; ${ }^{13}$ in which the flux shape is assumed to remain constant throughout a transient, the adiabatic method ${ }^{14}$ and its more sophisticated cousin, the quasistatic method, ${ }^{15}$ the nodal methods, ${ }^{16}$ and the modal expansion methods.17-19 These methods are all closely related, as will be shown in Chapters III, IV, and V. They all can be thought of as applications of the method of weighted residuals ${ }^{20}$ to the time-dependent diffusion equations.

In the weighted-residual procedure, the flux is expanded into a linear combination of known spatially dependent functions called trial functions. The approximate solution is then inserted into Eqs. 1.1 and 1.2. The resulting "residual" is then multiplied by appropriate weighting functions, and spatial integrations are carried out to determine the time-dependent functions. The method of weighted residuals is described in detail in Chapter II.

The unification of the various methods that treat the spatial dependence of the flux during a transient will not be made merely for pedantic reasons. Reactor physicists have a genuine need to known which approximations are suitable for particular problems. Unifying the methods enables the strengths and weaknesses of each to be assessed in a systematic manner. Therefore, a major goal of this report is to show as precisely as possible the similarities and differences among these weighted-residual methods. The methods differ in principle from one another only in the choices of the trial solutions.

Each method reflects, to a greater or lesser extent, all the fundamental advantages and limitations of trial-function methods. The methods all attempt to use results previously found, i.e., the trial functions, to predict the solution to the problems to which they are applied. Their success depends largely upon how carefully the trial functions are chosen. Experience and intuition are important factors in this respect. Furthermore, great care must be taken to ensure that the intuitive knowledge is applied only within the parameter range where it is valid. As of now, even the selection of this parameter range is largely intuitive. Therefore, alternative methods of solving the problems must remain available for checking purposes. Direct numerical solutions, inefficient and time-consuming as they may be; should provide this alternative. 
Another goal of this report is to compare different types of weightedresidual criteria in an attempt to determine the influence of the weighting functions on the success of the approximations. Previous investigators ${ }^{20-22}$ of weighted-residual methods have assigned far more importance to the choice of the trial functions than to the choice of weighting functions. However, the influence of weighting functions on the accuracy of the approximate space-time solutions remains to be seen.

Chapter II contains an outline of the method of weighted residuals. Chapter III contains a derivation of the multimode kinetics equations 23,24 using the method of weighted residuals. The multimode kinetics equations are the set of first-order ordinary differential equations common to all the approximate methods listed above. The point-reactor model and spatially continuous modal-expansion methods are also discussed in Chapter III as weighted-residual methods. The adjoint problem ${ }^{25,26}$ and the corresponding variational formulation are presented at the close of Chapter III.

Chapter IV is concerned with the possibilities for choosing spatially discontinuous trial functions. ${ }^{27,28}$ A technique known as multichaninel synthesis ${ }^{27}$ is described, as are the nodal methods. Temporally discontinuous trial functions ${ }^{29,30}$ are discussed in Chapter $V$ as choices to improve accuracy. The adiabatic and quasistatic methods are examples of this technique.

In Chapter VI, the multimode kinetics equations are integrated using the method of undetermined parameters. ${ }^{20}$ Piecewise polynominal functions $^{31}$ are chosen as trial solutions. Possible choices of weighting functions are also presented. An iterative scheme, ${ }^{32}$ which uses the method of weighted residuals, is applied to the space-time problem in Chapter VII. A simple illustration is made, and indications are given as to how it can be used with multichannel synthesis and temporally discontinuous synthesis.

Chapter VIII contains the results of some numerical studies along with interpretations of them. Emphasis is placed upon the choice of weighting functions when continuous trial functions are used. Also, nodal methods and multichannel synthesis are studied. One-group diffusion theory is used in all cases. Exact solutions are obtained with the computer program WIGLE. ${ }^{2}$

Conclusions and recommendations for further study are made in Chapter IX. Linear dependence is discussed in Appendix A. Appendix B contains a description of the time-step halving procedure used to obtain stable solutions of the multimode kinetics equations. 
CHAPTER II

THE METHOD OF WEIGHTED RESIDUALS

A. General Discussion

The method of weighted residuals is an appealing mathematical tool, because of both its wide utility and its ease in formulation. It not only handles the usual linear self-adjoint problems, but can also solve even the most.highly nonlinear and non-self-adjoint systems. The resulting approximate solutions are more or less "good," depending upon the experience and/or intuition of the human being using the method; this somewhat vague statement will. be made clearer below.

Consider a system of (generally) nonlinear partial differential equations in several independent variables, represented by the vector $\underline{x}$. This system of equations is $20,22,33$

$$
H \phi(\underline{x})=f(\underline{x}),
$$

and

$$
\mathrm{B}_{\mathrm{i}} \phi(\underline{\mathrm{x}})=\mathrm{g}_{\mathrm{i}}(\underline{\mathrm{x}}), \quad \mathrm{i}=1, \ldots, \mathrm{p},
$$

where $\mathrm{H}(\phi)$ is a nonlinear differential operator, $\phi(\underline{\mathbf{x}})$ is the vector of dependent variables, $f(\underline{x})$ and $g_{i}(\underline{x})$ are vectors containing functions of the independent variables, and the $B_{i}$ are operators representing the boundary conditions.

The system of equations represented by Eqs. 2.1 and 2.2 usually is solved either numerically or approximately. To apply the method of weighted residuals, an approximate solution is first sought in the form

$$
\bar{\phi}(\underline{\mathbf{x}})=\psi_{0}+\sum_{j=1}^{\mathrm{n}} \mathrm{A}_{\mathrm{j}} \psi_{\mathrm{j}}
$$

The functions $\psi_{0}$ and $\psi_{j}$ are trial solutions, chosen beforehand. In principle, these functions are arbitrary, but great care must be taken to ensure that they are all linearly independent. They should also satisfy the boundary conditions given by Eq. 2.2, preferably by choosing $\psi_{0}$ and $\psi_{\mathrm{j}}$ such that

and

$$
\left.\begin{array}{l}
B_{i}\left(\psi_{0}\right)=g_{i}, \quad i=1, \ldots, p, \\
B_{i}\left(\psi_{j}\right)=0, \quad j=1, \ldots, n,
\end{array}\right\}
$$

More will be said about boundary conditions in Section $C$ of this chapter. 
The trial functions in Eq. 2.3 are generally functions of all or all but one of the independent variables. The $A_{j}$ are either undetermined parameters or undetermined functions of one or more of the independent variables. Once determined, the $A_{j}$ 's will in turn provide an approximate solution to Eqs. 2.1 and 2.2 .

Since Eq. 2.3 is an approximation, it generally does not satisfy Eq. 2.1. A measure of the extent to which $\bar{\phi}$ satisfies Eq. 2.1 is given by the equation residual $R(A, \psi)$, which is formed when Eq. 2.3 is substituted into Eq. 2.1:

$$
R(A, \psi)=f-H \bar{\phi}=f-H\left(\psi_{0}+\sum_{j=1}^{n} A_{j} \psi_{j}\right) .
$$

As the number of approximating functions increases, the residuals decrease if the trial functions are "reasonable." If the residual is identically zero, the exact solution is one of the trial functions. If the residual does not vanish, a reasonable alternative is to make it small in some sense. The weighted-residual method provides this alternative. That is, n weighted averages of the residual are set equal to zero:

$$
\int_{D} w_{k} R d D=0, \quad k=1, \ldots, n .
$$

The independent variables in $D$ include all the independent variables upon which the $A_{j}$ do not depend.

If the $A_{j}$ are undetermined parameters, $n$ algebraic equations result, which may be solved for the $A_{j}$. When the $A_{j}$ are undetermined functions, Eq. 2.6 yields $n$ simultaneous differential equations. The method of undetermined parameters is often called the direct method; the method of undetermined functions is also known as the semidirect method, or-the Kantorovich method. ${ }^{34}$

Allowing the weighted averages of the residual to vanish distributes the errors over the entire range of integration. The errors are then said to be minimized in a certain sense. This "sense" coincides with whichever choices of weighting functions are made.

This closeness is strongly dependent upon the choice of trial functions, and less strongly upon the choice of weighting functions. In general, a good set of trial functions is obtained only by experience and by familiarity with the type of problem to be solved. The choice of weighting functions has been, in the past, more restricted. Several common types are used. These will be described next. 
B. Weighted-residual Criteria

Far less emphasis has traditionally been given to the choice of "good" weighting functions than to the choice of "good" trial functions. Such a question as "Do the weighting functions satisfy the boundary conditions?" is often not even asked, because it is not clear whether or not such a question is relevant. Nevertheless, there have been several attempts over the years to compare different weighted-residual methods. Notable among these are the works of Bickley in $1941,{ }^{35}$ and Collings in $1961 .^{36}$ Bickley solved a linear partial differential equation analogous to the heatconduction equation; Collings solved both linear and nonlinear problems in heat conduction. Both concluded that the Galerkin method of weighting (described in Section B.5 below) gave better results than the other methods used in their comparisons. Other investigator $\mathrm{s}^{20,37,38}$ have also commented on the apparent superiority of either the Galerkin method for self-adjoint systems, or variational methods for non-self-adjoint systems. It must be emphasized, however, that the results of these investigations do not constitute universal proof.

The following is a list of the most commonly used weighted-residual criteria.

1. The Method of Collocation ${ }^{20,22,33}$

The residual $R$ is allowed to vanish at $n$ points, $p=p_{1}, \ldots, p_{n}$ in D. The weighting functions are the Dirac delta functions

$$
\mathrm{W}_{\mathrm{k}}=\delta\left(\mathrm{p}-\mathrm{p}_{\mathrm{k}}\right), \quad \mathrm{k}=1, \ldots, \mathrm{n},
$$

so that Eq. 2.6 becomes

$$
\int_{D} \delta\left(p-p_{k}\right) R d D=R\left(p_{k}\right)=0, \quad k=1, \ldots, n \text {. }
$$

Equation 2.1 is then satisfied exactly at the $n$ collocation points. As $n$ is increased, the residual vanishes at more and more points and presumably decreases throughout $D$. The points $p_{k}$ are generally chosen in a uniform pattern.

2. The Method of Subdomains $20,22,33$

The domain $\mathrm{D}$ is divided into $\mathrm{n}$ distinct subdomains, $D_{1}, \ldots, D_{n}$, which may partially overlap: The weighting functions are unit functions in each subdomain; that is, 


$$
\left.\begin{array}{l}
\mathrm{w}_{\mathrm{k}}\left(D_{\mathrm{k}}\right)=1, \\
\mathrm{w}_{\mathrm{k}}\left(D_{\mathrm{j}}\right)=0, \quad \mathrm{k} \neq \mathrm{j} .
\end{array}\right\}
$$

In this case, Eq. 2.6 becomes

$$
\int_{D_{k}} R d D=0, \quad k=1, \ldots, n
$$

Equation 2.1 is then satisfied in an average manner in each of the $\mathrm{n}$ subdomains $D_{k}$. As the number of subdomains increases, Eq. 2.1 is satisfied in smaller and smaller regions, causing the residual to approach zero.

3. The Method of Least Squares ${ }^{20,22,33,39}$.

The integral of the square of the residual is minimized with respect to the undetermined parameters $\mathrm{A}_{\mathrm{j}}$ :

$$
\frac{\partial}{\partial A_{k}} \int_{D} R^{2} d D=2 \int_{D} \frac{\partial R}{\partial A_{k}} R d D=0, \quad k=1, \ldots, n
$$

The weighting functions are therefore

$$
\mathrm{w}_{\mathrm{k}}=\frac{\partial \mathrm{R}}{\partial \mathrm{A}_{\mathrm{k}}}, \quad \mathrm{k}=1, \ldots, \mathrm{n} .
$$

4. The Method of Moments ${ }^{22,33}$

The weighting functions are given by

$$
\mathrm{W}_{\mathrm{k}}=\mathrm{P}_{\mathrm{k}}(\mathrm{D}), \quad \mathrm{k}=1, \ldots, \mathrm{n},
$$

where the $P_{k}(D)$ are polynomials orthogonal to one another within $D$. When these weighting functions are used, i.e., when

$$
\int_{D} P_{k} R d D=0, \quad k=1, \ldots, n
$$

the first $n$ moments of $R$ vanish. Often, in one space dimension, the choice

$$
\mathrm{w}_{\mathrm{k}}(\mathrm{x})=\mathrm{x}^{\mathrm{k}}, \quad \mathrm{k}=1, \ldots, \mathrm{n},
$$


is called the method of moments, although these functions are not orthogonal.

$$
\text { 5. The Galerkin Method } 20,22,33
$$

The weighting functions are the trial functions themselves, so that Eq. 2.6 is

$$
\int_{D} \psi_{k} \mathrm{R} d \mathrm{D}=0, \quad \mathrm{k}=1, \ldots, \mathrm{n}
$$

If the trial functions are members of a complete set of functions, the Galerkin method makes the residual orthogonal to members of this complete set. If the operator $H$ is linear, and if the residual $R$ is or thogonal to every member of the complete set, then the residual would be identically zero. Thus, for this case, as the number of trial functions increases, the residual tends to vanish, so that convergence is guaranteed. In practice, however, convergence may be very slow. Currently, far more emphasis is placed upon a pragmatic choice of suitable trial functions than upon constructing a complete set of functions.

$$
\text { 6. Variational Methods }{ }^{39,40} \text {. }
$$

"A variational description of a physical system consists of a statement that the variation, or functional differential, of a specified functional is equal to some fixed value, which can be and customarily is chosen to be zero." 40 This statement also implies that one or more "Euler equations," along with their "natural boundary conditions" and initial conditions, exist. If these equations and conditions match the system given by Eqs.2.1 and 2.2, then the variational principle is an alternate way of formulating the problem. The functional is then said to be stationary with respect to arbitrary variations in the functions represented by $\phi(\underline{x})$, and with respect to whichever constraint conditions are present. (These must also be accounted for in the functional.)

Once a variational principle has been found, the so-called variational method can be used to effect an approximate solution to the system. The variational method substitutes a trial solution into the functional in the same manner as was done in the method of weighted residuals. In fact, as far as the choosing of trial functions is concerned, variational methods offer no greater insight than do weighted-residual methods. Indeed, it is well known that, for self-adjoint systems, the Galerkin method is equivalent to the variational method. ${ }^{39,40}$

A true variational principle yields additional information, which weighted-residual methods cannot. The functional may represent a quantity 
of importance; variational methods approximate this quantity more accurately than they approximate $\phi(\underline{\mathbf{x}})$.

The adjoint function $\phi^{+}$must appear in the functional when a variational principle is formulated for a non-self-adjoint system. ${ }^{39}$ Furthermore, the functional is not a true variational principle unless the adjoint system can be formed when variations are taken with respect to $\phi$. If the system is nonlinear, the variational principle must also be capable of generating any auxiliary equations which may be causing the nonlinearity.

problem ${ }^{39}$

To illustrate the use of a variational principle, consider the

$$
H \phi-f=0 \text {. }
$$

The following functional (which could possibly be physically important) can be determined quite accurately by taking arbitrary variations with respect to $\phi^{+}$:

$$
F=\int_{a}^{b} \phi^{+}(H \phi-f) d x
$$

where $\phi^{+}$is the function that satisfies the adjoint equation

$$
\mathrm{H}^{+} \phi^{+}-\mathrm{f}^{+}=0 \text {. }
$$

Now approximate $\phi$ by

$$
\bar{\phi}(\underline{x})=\sum_{k=1}^{n} A_{k} \psi_{k}(\underline{x})
$$

and $\phi^{+}$by

$$
\bar{\phi}^{+}(\underline{x})=\sum_{k=1}^{n} A_{k}^{+} \psi_{k}^{+}(\underline{x}) .
$$

The variational principle

$$
\int_{a}^{b} \delta \phi^{+}(H \phi-f) d x=0
$$

yields the set of equations 


$$
\int_{a}^{b} \psi_{k}^{+}(\underline{x})[H \bar{\phi}(\underline{x})-f] d x=0, k=1, \ldots, n,
$$

which in turn can be solved simultaneously to determine the $A_{k}$. Equation 2.23 is recognized as being of the same form as Eq. 2.6, where

$$
\mathrm{W}_{\mathrm{k}}(\underline{\mathrm{x}})=\psi_{\mathrm{k}}^{+}(\underline{\mathrm{x}})
$$

Now, of course, the functions $\psi_{\mathrm{k}}^{+}(\mathrm{x})$ must also be chosen beforehand, as they are."trial functions" used in the approximate solution to the adjoint problem, Eq. 2.19. These functions are commonly chosen to be the same as the functions $\psi_{\mathrm{k}}(\underline{\mathrm{x}})$, so that the method represented by Eqs. 2.23 and 2.24 is identical to the Galerkin method. If the system is self-adjoint, then $\mathrm{A}_{\mathrm{k}}^{+} \equiv \mathrm{A}_{\mathrm{k}}$ for all $\mathrm{k}$; if the system is non-self-adjoint, then the coefficients will differ, and separate solutions to the real and to the adjoint problem will be obtained. In either case, the functional $F$ will be determined accurately if good'trial functions are chosen. For the non-self-adjoint system, the variational method is equivalent to solving two problems simultaneously by using the method of weighted residuals. The trial functions for the adjoint problem act as the weighting functions for the "physical" problem, and vice versa.

C. Initial Conditions and Boundary Conditions

The approximate solution given by Eq. 2.3 will not, in general, satisfy the initial conditions in an initial-value problem. To obtain initial values of the $A_{j}$, when they are undetermined functions of time, an initial residual is formed, which is weighted in the same manner as the differential equation. The initial values of $A_{j}$ then serve as initial conditions for the $A_{j}(t)$ when the method of undetermined functions is used.

Often the trial functions satisfy neither the differential equation nor the boundary conditions. The boundary conditions should then be weighted by the same set of weighting functions as the differential equation. ${ }^{41}$. Surface integrals should be carried out on these weighted boundary residuals, and added to the differential-equation weighted residuals. The resulting weighted residual is then allowed to vanish in the usual manner. The resulting equation, interestingly enough, looks much like the first variation of a functional that has been used in a variational principle.

This technique of combining equation residuals and boundary residuals is an invaluable way to treat systems of equations in which the equations are related through boundary conditions. An outstanding example of a system of equations that are related in this manner is the multigroup, multiregion formulation of neutron-diffusion theory, in which the neutron fluxes 
and currents are assumed to be continuous at interfaces between adjacent regions. A detailed development is made in Chapter IV to obtain the multichannel-s.ynthesis formulation.

D. Some Observations on Accuracy

The motivation behind using a weighted-residual method, as opposed to using another approximate method, such as Taylor-series expansions or perturbation techniques, is to distribute the error as uniformly as possible over the range of the independent variables. ${ }^{22}$ Taylor-series expansions and perturbation techniques yield highly accurate results near the initial state, but may not work so well over most of the range. Weighted-residual methods, being error-distribution principles, tend to sacrifice high initial accuracy in order to obtain reasonable accuracy throughout. Unfortunately, a proper choice of trial functions is essential, and the selection is not always easy.

Another unfortunate circumstance is that realistic error bounds are rarely obtained from weighted-residual procedures. ${ }^{22}$ There are, however, convergence theorems available for certain classes of problems. ${ }^{41-43}$ Most of these apply to the Galerkin method. However, even with the assurance that a convergent solution will be obtained by decreasing the increment or iterating indefinitely, the convergence may be so slow that the method becomes computationally impractical unless good trial functions are used.

The usual way of testing a weighted-residual method is to apply it to a problem whose exact solution is already known. If the errors are satisfactorily small with a given choice of trial functions, then the method is used, with the same set of trial functions, on other problems of the same type.

E. An Iterative Technique to Improve Accuracy

Up to now it has been repeatedly pointed out that a proper choice of trial functions is essential to the success of any weighted-residual method. To overcome this weakness, Kerr ${ }^{32}$ developed a rapidly converging iterative technique, which itself strongly uses the method of weighted residuals. This technique extends the method of undetermined functions in a most ingenious and logical manner.

When the trial solution given by Eq. 2.3 is postulated, where the $A_{j}$ are undetermined functions, the $A_{j}$ are usually chosen to be functions of only one of the independent variables, say $x_{1}$. The trial solutions $\psi_{j}$ are generally chosen to be functions of the remaining members of $\underline{x}$; that is,

$$
\psi_{j}=\psi_{j}\left(x_{2}, \ldots, x_{r}\right) \equiv \psi_{j \mathfrak{l}}^{\mathfrak{l}}\left(\mathbf{x}_{2}, \ldots, x_{r}\right)
$$


when

$$
\underline{x}=\left(x_{1}, \ldots, x_{r}\right)
$$

A weighted-residual criterion will produce a set of ordinary differential equations to be solved for the $A_{j}\left(x_{1}\right)$. Limited by the initial choice of the $\psi_{j}$ 's, the approximation nevertheless yields a solution that tends toward the exact solution along the $\mathbf{x}_{1}$ direction. Kerr decided that the solution could be further improved along the directions of the remaining independent variables as follows:

After having determined the $A_{j}\left(x_{1}\right)$, relabel them as $A_{j 1}^{1}\left(x_{1}\right)$ and let

$$
\psi_{j 1}^{1}\left(x_{2}, \ldots, x_{r}\right)=A_{j 2}^{1}\left(x_{2}\right) \psi_{j 2}^{1}\left(x_{3}, \ldots, x_{r}\right),
$$

where the $\psi_{\mathrm{j} 2}^{1}$ are also chosen beforehand. Equation 2.25 is substituted back into Eq. 2.3, which becomes (neglecting $\psi_{0}$ )

$$
\bar{\phi}(\underline{x})=\sum_{j=1}^{n} A_{j 1}^{1}\left(x_{1}\right) A_{j 2}^{1}\left(x_{2}\right) \psi_{j 2}^{1}\left(x_{3}, \ldots, x_{r}\right)
$$

To determine the $A_{j_{2}}^{1}\left(x_{2}\right)$, use a weighted-residual criterion with respect to $\left(\mathrm{x}_{1}, \quad, \mathrm{x}_{3}, \ldots, \mathrm{x}_{\mathrm{r}}\right)$. Then, let

$$
\psi_{\mathrm{j}_{2}}^{1}\left(\mathrm{x}_{3}, \ldots, \mathrm{x}_{\mathrm{r}}\right)=\mathrm{A}_{\mathrm{j}_{3}}^{1}\left(\mathrm{x}_{3}\right) \psi_{\mathrm{j}_{3}}^{1}\left(\mathrm{x}_{4}, \ldots, \mathrm{x}_{\mathrm{r}}\right) .
$$

Again, substitute Eq. 2.27 into Eq. 2.3, and use a weighted-residual criterion with respect to $\left(x_{1}, x_{2}, \quad, x_{4}, \ldots, x_{r}\right)$ to determine the $A_{j_{3}}^{l}\left(x_{3}\right)$.

This process is continued until all the functions $A_{j_{1}}^{1}\left(x_{1}\right), \ldots, A_{j r}^{1}\left(x_{r}\right)$ have been determined. Then, the approximate solution is given by

$$
\bar{\phi}^{1}(\underline{x})=\sum_{j=1}^{n} A_{j 1}^{1}\left(x_{1}\right) A_{j 2}^{1}\left(x_{2}\right) \ldots A_{j r}^{1}\left(x_{r}\right) .
$$

At this stage, a second iteration is begun; the improved expressions for $A_{j_{1}}\left(x_{1}\right)$, now labeled $A_{j_{1}}^{2}\left(x_{1}\right)$, can be found by using the $n$ products $A_{j 2}^{1}\left(x_{2}\right) A_{j 3}^{1}\left(x_{3}\right) \ldots A_{j r}^{1}\left(x_{r}\right)$ as "trial functions. "Then, $A_{j 2}^{2}\left(x_{2}\right)$ are found, using the products $A_{j 1}^{2}\left(x_{1}\right) A_{j 3}^{1}\left(x_{3}\right) \ldots A_{j r}^{1}\left(x_{r}\right)$ as trial functions, and so on, until the second iteration has been completed. At this point, the approximate solution is

$$
\bar{\phi}^{2}(\underline{x})=\sum_{j=1}^{n} A_{j 1}^{2}\left(x_{1}\right) A_{j 2}^{2}\left(x_{2}\right) \ldots A_{j r}^{2}\left(x_{r}\right) .
$$


More iterations can be performed, but Kerr found that very few cycles are needed to obtain a converged solution. In addition, and more importantly, he showed that the final form of the generated solution is independent of the initial choice of the $\psi_{j}{ }^{\prime} s$. The accuracy of the solution depends only upon the choices of weighting functions and on the number of trial functions chosen.

The advantage of choosing arbitrary trial functions is only at the expense of increased computational effort. The iterative technique could be time-consuming, even using a computer. In addition, the re is still some advantage to choosing reasonable trial solutions. Kerr has indicated that by. choosing trial solutions that satisfy the boundary conditions, he could reduce the number of iterations needed for convergence. In summary, the method is potentially very powerful and very accurate. The formulation for the time-dependent diffusion equations is developed in Chapter VII. 


\section{CHAP TER III}

\section{APPROXIMATE SOLUTION OF THE TIME- DEPENDENT DIFFUSION EQUATIONS}

In this chapter, the weighted-residual method is used to solve the time-dependent multigroup diffusion equations. In particular, the method of undetermined functions is used to derive the set of equations known as the multimode kinetics equations. ${ }^{1}$ Then, the point-reactor model and modal-expansion approximations are discussed.within the framework of this method. Finally, the adjoint problem ${ }^{1}$ is formulated to show how a variational principle can be used. The treatment is for a one-dimensional slab reactor, both in this chapter and in the remainder of this report. Extension to different geometries and to more than one dimension is straightforward.

A. The Method of Undetermined Functions

For a one-dimensional slab geometry, the multigroup diffusion equations and precursor equations can be written as

$$
\begin{aligned}
\mathrm{V}^{-1} \frac{\partial \phi(\mathrm{x}, \mathrm{t})}{\partial \mathrm{t}}= & {\left[\nabla \cdot \mathrm{D}(\mathrm{x}, \mathrm{t}) \nabla-\mathrm{A}(\mathrm{x}, \mathrm{t})+(1-\beta) \chi_{\mathrm{p}} \mathrm{F}^{\mathrm{T}}(\mathrm{x}, \mathrm{t})\right](\mathrm{x}, \mathrm{t}) } \\
& +\sum_{j=1}^{\mathrm{J}} \lambda_{\mathrm{j}} \chi_{\mathrm{j}} \eta_{\mathrm{j}}(\mathrm{x}, \mathrm{t}),
\end{aligned}
$$

and

$$
\frac{\partial \eta_{j}}{\partial t}(x, t)=\beta_{j} F^{T}(x, t) \phi(x, t)-\lambda_{j} \eta_{j}(x, t), \quad j=1, \ldots, J
$$

where the meanings of the symbols are given by Eqs. 1.3-1.10. The matrices $D, A$, and $F^{T}$ can be functions of time. In fact, they could even be functionals of the flux vector $\phi(x, t)$. If so, the system would be nonlinear.

The method of undetermined functions proceeds by expanding the group fluxes, given by Eq. 1.3, in the trial functions (or modes) $\psi_{i}(x, t)$, as follows:

$$
\bar{\phi}(\mathrm{x}, \mathrm{t})=\sum_{\mathrm{i}=1}^{\mathrm{I}} \psi_{\mathrm{i}}(\mathrm{x}, \mathrm{t}) \mathrm{N}_{\mathrm{i}}(\mathrm{t}) \equiv \psi(\mathrm{x}, \mathrm{t}) \mathrm{N}(\mathrm{t})
$$

The bar over the flux vector indicates that Eq. 3.3 is an approximate solution. The modes $\psi_{j}(x, t)$ are known as shape or trial functions; the undetermined functions $N_{i}(t)$ are called amplitude functions. ${ }^{23}$ Most of the time 
dependence is carried by the amplitude functions: The choice of the trial functions is in principle arbitrary, except for the condition that they must be linearly independent.

In Eq. 3.3, the modes $\psi_{\mathrm{i}}(\mathbf{x}, \mathrm{t})$ are chosen to be the column vectors

$$
\psi_{i}(x, t)=\left[\begin{array}{c}
\psi_{1 i}(\dot{x}, t) \\
\cdot \\
\cdot \\
\psi_{\mathrm{Gi}}(\mathrm{x}, \mathrm{t})
\end{array}\right], \quad i=1, \ldots, \mathrm{I}
$$

and are combined to form the G $\mathrm{x}$ I matrix of trial functions $\psi(\mathrm{x}, \mathrm{t})$ in the following manner:

$$
\begin{aligned}
\psi(\mathrm{x}, \mathrm{t}) & =\left[\psi_{\mathrm{I}}(\mathrm{x}, \mathrm{t}) \ldots \psi_{\mathrm{I}}(\mathrm{x}, \mathrm{t})\right] \\
& =\left[\begin{array}{ccc}
\psi_{11}(\mathrm{x}, \mathrm{t}) & \cdots & \psi_{1 I}(\dot{x}, \mathrm{t}) \\
\vdots & \cdots & \vdots \\
\vdots & \cdots & \cdot \\
\psi_{\mathrm{Gi}}(\mathrm{x}, \mathrm{t}) & \cdots & \psi_{\mathrm{GI}}(\mathrm{x}, \mathrm{t})
\end{array}\right] .
\end{aligned}
$$

Each amplitude function $N_{i}(t)$ is a scalar function, so that $N(t)$ is a column vector of amplitude functions:

$$
N(t)=\left[\begin{array}{c}
N_{1}(t) \\
\cdot \\
\cdot \\
N_{I}(t)
\end{array}\right]
$$

The next step is to substitute the approximate solution Eq. 3.3 into Eqs. 3.1 and 3.2 to obtain

$$
\begin{aligned}
\mathrm{V}^{-1} \psi(\mathbf{x}, \mathrm{t}) \frac{\mathrm{dN}}{\mathrm{dt}}= & {\left[\nabla \cdot \mathrm{D}(\mathrm{x}, \mathrm{t}) \nabla-\mathrm{A}(\mathrm{x}, \mathrm{t})+(1-\beta) \chi_{\mathrm{p}} \mathrm{F}^{\mathrm{T}}(\mathrm{x}, \mathrm{t})-\mathrm{V}^{-1} \frac{\partial}{\partial \mathrm{t}}\right] \psi(\mathrm{x}, \mathrm{t}) } \\
& +\sum_{\mathrm{j}=1}^{\mathrm{J}} \lambda_{\mathrm{j}} \chi_{\mathrm{j}} \eta_{\mathrm{j}}(\mathrm{x}, \mathrm{t})
\end{aligned}
$$

and

$$
\frac{\partial \eta_{j}}{\partial t}(x, t)=\beta_{j} F^{T}(x, t) \psi(x, t) N(t)-\lambda_{j} \eta_{j}(x ; t)
$$


The above expressions are not truly equations unless the exact solution happens to be contained within the approximate solution. The approximation is more properly expressed by subtracting the right-hand sides of each from the left-hand sides to form the following residuals:

$$
\begin{aligned}
R_{F}(x, t)= & \cdot V^{-1}\left[\psi(x, t) \frac{d N}{d t}+\frac{\partial \psi}{\partial t} N(t)\right]-[\nabla \cdot D \nabla-A \\
& \left.+(1-\beta) \chi_{p} F^{T}\right] \psi(x, t) N(t)-\sum_{j=1}^{J} \lambda_{j} \chi_{j} \eta_{j}(x, t),
\end{aligned}
$$

and

$$
\mathrm{R}_{\mathrm{P}_{\mathrm{j}}}(\mathrm{x}, \mathrm{t})=\frac{\partial \eta_{\mathrm{j}}}{\partial \mathrm{t}}(\mathrm{x}, \mathrm{t})-\beta_{\mathrm{j}} \mathrm{F}^{\mathrm{T}} \psi(\mathrm{x}, \mathrm{t}) \mathrm{N}(\mathrm{t})+\lambda_{\mathrm{j}} \eta_{\mathrm{j}}(\mathrm{x}, \mathrm{t})
$$

The residuals $R P_{j}(x, t)$ given by Eq. 3.10 are formed so that the multimode kinetics equations can be obtained. This procedure is not necessary, however. An alternate way to solve the space-time problem is to formally integrate Eq. 3.2 with respect to the time variable (see Eq. 7.1), and substitute the result into Eq. 3.1 .

In addition to obeying Eqs. 3.1 and 3.2 , the group fluxes must also satisfy the following initial and boundary conditions:

$$
\phi(x, 0)=\phi_{0}(\mathbf{x})=\left[\begin{array}{c}
\phi_{10}(x) \\
\cdot \\
\cdot \\
\cdot \\
\phi_{\mathrm{G}_{0}}(\mathrm{x})
\end{array}\right]
$$

and

$$
\phi(\mathrm{a}, \mathrm{t})=\phi(\mathrm{b}, \mathrm{t})=0,
$$

where $a$ and $b$ are the outer boundaries of the reactor. When the trial solution Eq. 3.3 is substituted into Eq. 3.11, the following initial residuals result:

$$
\mathrm{R}_{\mathrm{I}}(\mathrm{x})=\phi_{0}(\mathrm{x})-\psi(\mathrm{x}, 0) \mathrm{N}(0)
$$

A common procedure is to choose one of the $\psi_{i}$ vectors to be the initial state $\phi_{0}(x)$. Then, the corresponding amplitude function $N_{i}(0)$ is completely determined (usually chosen to be unity), while the remaining amplitude functions are initially zero. 
The simplest way to ensure that the trial solution satisfies the boundary conditions is to choose only trial functions that satisfy these boundary conditions. Failing this, the following boundary residuals are formed:

$$
R_{A}(t)=\psi(a, t) N(t),
$$

and

$$
R_{B}(t)=\psi(b, t) N(t)
$$

The residuals given by Eqs. 3.9, 3.10,3.13,3.14, and 3.15 vanish identically only when the exact solution is contained within the approximate solution. Otherwise, they are nonzero functions of space and time. As yet, they cannot be evaluated because the functions $N_{i}(t)$ have not yet been determined. A good approximation prociedure should yield functions $N_{i}(t)$ which minimize the residuals in some manner. One such procedure, the method of weighted residuals, will now be used to derive the multimode kinetics equations. The multimode kinetics equation can in turn be solved to complete the approximate solution to Eqs. 3.1 and 3.2.

B. The Multimode Kinetics Equations

Consider the G $x$ I matrix of weighting functions

$$
W(x, t)=\left[\begin{array}{ccc}
w_{11}(x, t) & \ldots & w_{1 I}(x, t) \\
\cdot & \cdots & \cdot \\
\cdot & \cdots & \cdot \\
w_{G 1}(x, t) & \cdots & w_{G I}(x, t)
\end{array}\right],
$$

which, in principle, can be chosen arbitrarily. It must, however, be of the same order as the matrix of trial functions Eq. 3.5. In addition, no two columns can be proportional to one another, or a linearly dependent system will result. With the use of $\mathrm{Eq}$. 3.16, the method of weighted residuals can be applied as follows.

The boundary residuals $R_{A}(t)$ and $R_{B}(t)$ can be combined with the differential-equation residuals $R_{F}(x, t)$ as follows: Multiply Eq. 3.9 by Eq. 3.16, and integrate over the spatial variable: The boundary.residuals $R_{A}(t)$ and $R_{B}(t)$ are then multiplied by $W(a, t)$ and $W(b, t)$, respectively. The boundary weighted residuals are then subtracted from the differential- :" equation weighted residuals, and the result allowed to vanish:

$$
\int_{x} W^{T}(x, t) R_{E}(x, t) d x=\int_{x} W^{T}(x, t) R_{F}(x, t) d x-W^{T}(a, t) R_{A}(t)-W^{T}(b, t) R_{B}(t),
$$


and

$$
\int_{x} w^{T}(x, t) R_{E}(x, t) d x=0
$$

In addition, Eq. 3.10 can be multiplied by $\mathrm{W}^{\mathrm{T}}(\mathrm{x}, \mathrm{t})$, and Eq. 3.13 can be multiplied by $\mathrm{W}^{\mathrm{T}}(\mathrm{x}, 0)$. The resulting weighted-residual criteria are

$$
\int_{x} W^{T}(x, t) R_{P_{j}}(x, t) d x=0
$$

and

$$
\int_{\mathbf{x}} \mathrm{W}^{\mathrm{T}}(\mathbf{x}, 0) \mathrm{R}_{\mathrm{I}}(\mathbf{x}) \cdot \mathrm{d} \mathbf{x}=0
$$

The weighted-residual criteria represented by Eqs. 3.18-3.20 yield a set of first-order ordinary differential equations, which can be solved to determine the unknown functions $\mathrm{N}_{i}(t)$. This set of equations can be put into a familiar form if the spatial integrals are appropriately defined. This will now be done.

Define a set of precursor amplitude functions by

$$
C_{j}(t)=\int_{x} w^{T}(x, t) \chi_{j} \eta_{j}(x, t) d x, \quad j=1, \ldots, J
$$

Assuming that the matrices $\chi_{j}$ are time-independent, we can differentiate Eq. 3.21 to obtain

$$
\frac{d C_{j}}{d t}=\int_{x} w^{T}(x, t) \chi_{j} \frac{\partial \eta_{j}}{\partial t} d x+\int_{x} \frac{\partial w^{T}}{\partial t} \chi_{j} \eta_{j}(x, t) d x
$$

For the amplitude functions to contain most of the time dependence, both $\psi(\mathbf{x}, \mathrm{t})$ and $\mathrm{W}(\mathrm{x}, \mathrm{t})$ should contain only slowly varying functions which are bounded for all $x$ and. $t$. One way of ensuring that these criteria are satisfied is to impose the constraint condition ${ }^{44}$

$$
\int_{\mathbf{x}} \cdot \mathrm{w}^{\mathrm{T}}(\mathbf{x}, \mathrm{t}) \mathrm{V}^{-1} \frac{\partial \psi}{\partial \mathrm{t}} \mathrm{dx}=0
$$


which will enable the multimode kinetics equations to be derived. If the shape functions are picked beforehand, they must be chosen such that Eq. 3.23 is not violated. If they are calculated during the transient analysis, Eq. 3.23 acts as a normalization condition on $\psi(x, t)$. If Galerkin weighting is used, Eq. 3.23 becomes

$$
\frac{1}{2} \frac{\partial}{\partial t} \int_{x} \psi^{T}(x, t) V^{-1} \psi(x, t) d x=0
$$

If, as is commonly the case, the trial functions and the weighting functions are chosen to be time-independent, the constraint conditions are satisfied automatically. Furthermore, the second term on the right-hand side of Eq. 3.22 vanishes. Henceforth, time dependence of the trial functions will be retained, but the weighting functions will be assumed to be timeindependent.

When Eqs. 3.21-3.23 are substituted into the weighted-residual criteria, Eqs. 3.18-3.20, the following equations are obtained:

$$
\begin{aligned}
& \int_{\mathbf{x}} \mathrm{W}^{\mathrm{T}}(\mathbf{x}) \mathrm{V}^{-1} \psi(\mathbf{x}, \mathrm{t}) \mathrm{d} \mathbf{x} \frac{\mathrm{dN}}{\mathrm{dt}}=\int_{\mathbf{x}} \mathrm{W}^{\mathrm{T}}(\mathbf{x})[\nabla: \mathrm{D} \nabla-\mathrm{A} \\
& +(1-\beta) \chi_{p^{2}} F^{T} \psi(x, t) d x N(t) \\
& +\sum_{j} \lambda_{j} C_{j}(t), \\
& \frac{d C_{j}}{d t}=\beta_{j} \int_{x} W^{T}(x) \chi_{j} F^{T} \psi(x, t) d x N(t)-\lambda_{j} C_{j}(t),
\end{aligned}
$$

and

$$
\int_{x} \mathrm{~W}^{\mathrm{T}}(\mathrm{x}) \phi_{0}(\mathrm{x}) \mathrm{dx}=\int_{\mathbf{x}} \mathrm{W}^{\mathrm{T}}(\mathrm{x}) \psi(\mathrm{x}, 0) \mathrm{dx} \mathrm{N}(0)
$$

The spatial integrals in Eqs. 3.25 and 3.26 can all be written in shorthand form by defining the following I $x$ I matrices:

$$
\Lambda(t)=\int_{\mathbf{x}} \mathrm{w}^{\mathrm{T}}(\mathrm{x}) \mathrm{V}^{-1} \psi(\mathrm{x}, \mathrm{t}) \mathrm{dx}
$$




$$
\begin{aligned}
& \rho(t)=\int_{\mathbf{x}} \mathrm{W}^{\mathrm{T}}(\mathbf{x})\left[\nabla \cdot \mathrm{D} \nabla-\mathrm{A}+(1-\beta) \chi_{\mathrm{p}} \mathrm{F}^{\mathrm{T}}\right. \\
& \left.+\sum_{j=1}^{J} \beta_{j} \chi_{j} F^{T}\right] \psi(x, t) d x \\
& \bar{\beta}_{j}(t)=\beta_{j} \int_{x} w^{T}(x) \chi_{j} F^{T} \psi(x, t) d x,
\end{aligned}
$$

and

$$
\bar{\beta}(t)=\sum_{j=1}^{J} \bar{\beta}_{j}(t)
$$

When Eqs. 3.28-3.31 are substituted back into Eqs. 3.25 and 3.26, we obtain the multimode kinetics equations ${ }^{23}$

$$
\Lambda(t) \frac{d N}{d t}=[\rho(t)-\bar{\beta}(t)] N(t)+\sum_{j=1}^{J} \lambda_{j} C_{j}(t),
$$

and

$$
\frac{d C_{j}}{d t}=\bar{\beta}_{j}(t) N(t)-\lambda_{j} C_{j}(t), \quad j=1, \ldots, J
$$

Finally, assume that $\psi_{\mathbb{I}}(\mathrm{x}, 0)=\phi_{0}(\mathrm{x})$, so that the initial conditions for the $\mathrm{N}_{\mathrm{i}}(\mathrm{t})$ become

$$
\left.\begin{array}{l}
N_{1}(0)=1, . \\
N_{i}(0)=0, \quad i=2, \ldots, I_{0}
\end{array}\right\}
$$

The multimode kinetics equations can thus be written in the same form as the equations for the point-reactor model. The matrices given by Eqs. 3.28-3.31 are called the generation-time matrix, the reactivity matrix; and the effective delayed-neutron-fraction matrices. Furthermore, as is seen in Section $C$ below, the point-reactor model is the simplest special case of the multimode kinetics formulation. Other special cases, which are also discussed in this chapter, include orthogonal-expansion methods ${ }^{17}$ and 
synthesis methods. ${ }^{18}$ Techniques that use either spatially ${ }^{27,28}$ or temporally 29,30 discontinuous trial functions, such as nodal methods or quasistatic methods, can also be considered in this context. Since they require slight modifications in the derivation, of their multimode kinetics equations, they are discussed in more detail in Chapters IV and V, respectively.

C. The Point-reactor Model

The point-reactor model ${ }^{13}$ was the earliest and simplest method introduced to treat reactor transients. Its name is derived from the assumption that the flux remains in the same spatial configuration throughout a transient; i.e., the reactor can be treated by considering only one point in space.

As a weighted-residual method, the point-reactor model corresponds to choosing just one time-independent mode in the expansion given by

Eq. 3.3; i.e.,

$$
\bar{\phi}(\mathrm{x}, \mathrm{t})=\psi_{1}(\mathrm{x}) \mathrm{N}_{1}(\mathrm{t})
$$

This mode is usually, but not always, chosen to be the initial flux distribution. In principle, any weighting functions can be chosen, but traditionally the steady-state adjoint functions have been used. ${ }^{45}$ The possibility of choosing other weighting functions has been considered by Gozani ${ }^{46}$ and by Gross and Marable, ${ }^{47}$ although neither reported any numerical comparisons.

The mathematical properties of the point-reactor model are well known. ${ }^{48}$ The amplitude function is expressed by a sum of $\mathrm{J}+1$ real-valued exponentials $\exp \left(\omega_{j} t\right), J$ of which have negative arguments. The remaining exponential has either a positive or a negative argument, depending upon whether the reactivity is positive or negative. The exponent that can change sign is identified with the so-called asymptotic period. Each of the $\omega_{j}$ is a root of the so-called inhour equation, given by

$$
\rho=\Lambda \omega+\sum_{j=1}^{J} \frac{\beta_{j} \omega}{\omega+\lambda_{j}} .
$$

Although the point-reactor model is not adequate to describe transients in large reactors, much has been learned about the nature of reactor transients from its use. The role of the delayed-neutron precursors can be well understood by using it. In fact, the value $\rho=\beta$ (prompt critical) is of vital importance. When $\rho<\beta$, the delayed neutrons arising from decay of fission products aid in controlling a reactor excursion. When $\rho>\beta$, this control feature vanishes. The point-reactor model and the concept of reactivity contain too much valuable physical information to be abandoned entirely. For this reason, the multimode kinetics equations are written as 
a generalization of the point-reactor model. Furthermore, in Chapter VIII, attempts are made, when using more sophisticated approximations, to interpret transients in terms of the terminology of the point-reactor model, such as reactivity, asymptotic period, and prompt critical.

D. Continuous Spatial Modes

In a linear problem, if the Galerkin method is used in conjunction with a complete set of trial functions, a convergent solution is guaranteed. ${ }^{33,41}$ This fact is well known in the theory of orthogonal functions and Fourier series. ${ }^{49}$ It was only natural, then, that an expansion into a series of orthogonal functions would be one of the first techniques studied. Such investigations were reported by Garabedian et al. ${ }^{17,50,51}$

Garabedian's criterion for choosing the trial functions is to choose, in order, each function that obeys the Helmholtz equation,

$$
\nabla^{2} \psi+\mathrm{B}^{2} \psi=0
$$

and satisfies the boundary conditions given by Eq.3.12. The resulting trial functions form a complete set and are given by

$$
\psi_{\mathrm{gi}}(\mathrm{x})=\cos \frac{(2 i-1) \pi x}{b-a}, \quad g=1, \ldots, \mathrm{G} ; \quad i=1, \ldots, \mathrm{I}
$$

This same set of functions also serves as. the set of weighting functions, so that Galerkin weighting is being used.

Unfortunately, however, Garabedian's method has not been widely adopted for two reasons. First, convergence is not guaranteed for systems in which the coefficients are time-deperident, or for nonlinear systems. In these cases, the method has lost any advantages it may have possessed over other criteria. Second, and more significantly, convergence has been found to be very slow for all but the most simple problems; many modes are needed to obtain a convergent solution. For these reasons, many investigators ${ }^{19,23,27}$ have turned to more pragmatic ways of choosing trial functions.

The so-called natural modes ${ }^{1,45}$ of the reactor have been suggested as trial-function choices. These are the modes that result when Eqs. 3.1 and 3.2 are put into the form of eigenvalue problems. The "lambda modes" are the solutions of the static eigenvalue problem

$$
[-\nabla \cdot D \nabla+A] \psi(x)=\frac{1}{\lambda}\left[(1-\beta) \chi_{p}+\sum_{j=1}^{J} \beta_{j} \chi_{j}\right] F^{T} \dot{\psi}(x)
$$


The "omega-p modes" 1 are the modes of a reactor with no delayed-neutron source. They are formed by replacing $\partial \phi / \partial t$ by $\omega_{p} \phi$, to obtain

$$
\left[\nabla \cdot \mathrm{D} \nabla-\mathrm{A}+(1-\beta) \chi_{\mathrm{p}} \mathrm{F}^{\mathrm{T}}\right] \psi(\mathrm{x})=\omega_{\mathrm{p}} \mathrm{V}^{-1} \psi(\mathrm{x})
$$

Finally, the "omega-d modes" ${ }^{1}$ are the natural modes of the reactor with delayed neutrons. As a result, they include not only the flux components $\psi(x)$, but also the delayed components $\xi_{j}(x)$, as is shown in

$$
\left[\nabla \cdot \mathrm{D} \nabla-\mathrm{A}+(1-\beta) \chi_{\mathrm{p}} \mathrm{F}^{\mathrm{T}}\right] \psi(\mathrm{x})+\sum_{\mathrm{j}=1}^{\mathrm{J}} \lambda_{\mathrm{j}} \chi_{\mathrm{j}} \xi_{\mathrm{j}}(\mathrm{x})=\omega_{\mathrm{d}} \mathrm{V}^{-1} \psi(\mathrm{x}),
$$

and

$$
\beta_{j} F^{T} \psi(x)-\lambda_{j} \xi_{j}(x)=\omega_{d} \xi_{j}(x)
$$

The natural modes exhibit the properties of finality ${ }^{52}$ and biorthogonality, ${ }^{53}$ that is, if the adjoint problem is formulated, and the adjoint functions are used as weighting functions (biorthogonality), the multimode kinetics equations will be decoupled (finality). Each amplitude function can then be determined independently. Unfortunately, the natural modes have little more than academic value because they are extremely difficult to generate. This is particularly true for the omega-d modes, which change whenever the shape changes; i.e., they are continually changing.

The modes in Eq. 3.3 do not have to be generated by methods that formulate eigenvalue problems. For example, Dougherty and Shen ${ }^{19}$ generate what they call "Green's function modes" by solving diffusion equations with fixed fission sources in various subregions of the reactor. Once they have determined the modes, they formulate a variational principle from which they determine the amplitude functions by using a semidirect method. They use the adjoint function in their variational principle, which in turn is approximated in the same manner as is the flux, i.e., by a linear combination of the functions adjoint to the "Green's function modes." As described in Chapter II, this technique of forming a variational method is a generalization of the method of weighted residuals. The trial functions of the adjoint problem serve as weighting functions in the flux system, and vice versa. The adjoint problem and the corresponding variational method for the spacetime problem are described in Section $F$ of this chapter.

E. Synthesis Modes

A synthesis method ${ }^{23}$ attempts to construct a solution to a multidimensional system from solutions of lower-dimensional systems. For example, the three-dimensional steady-state flux distribution in a nuclear reactor could be constructed by combining solutions to two-dimensional problems in a weighted-residual procedure. Or, with more relevance to 
the space-time problem, the trial functions in Eq. 3.5 could be chosen as actual shapes characteristic of possible states of the reactor being studied.

A "good" set of trial functions would consist of shapes that would in some way "bracket" the true flux distribution during a transient. This technique is especially valuable in the analysis of control-rod movements for routine operation. For example, the shape of the flux with all rods in, or with all rods out, is well known. These shapes do indeed bracket flux configurations that exist while the rods are moving.

The bracketing idea has dubious value in the analysis of highly nonlinear accident-induced transients. There is no way, in this case, to pick the bracketing shapes beforehand. Nevertheless, flux synthesis could still be used to analyze transients initiated by a wide variety of accidents. In particular, the choices of spatially and temporally discontinuous trial functions, which are discussed in Chapters IV and V, offer far more flexibility than the continuous synthesis presently under discussion.

F. The Adjoint Problem: A Variational Principle

When the system represented by Eqs. 3.1, 3.2, 3.11, and 3.12 is linear, there is a set of equations adjoint to it, which can be written as ${ }^{1}$

$-\mathrm{V}^{-1} \frac{\partial \phi^{*}}{\partial t}(\mathrm{x}, \mathrm{t})=\left[\nabla \cdot \mathrm{D} \nabla-\mathrm{A}^{\mathrm{T}}+(\mathrm{l}-\beta) F \chi_{\mathrm{p}}^{\mathrm{T}}\right] \phi^{*}(\mathrm{x}, \mathrm{t})+\sum_{\mathrm{j}=1}^{\mathrm{J}} \beta_{\mathrm{j}} \eta_{\mathrm{j}}^{*}(\mathrm{x}, \mathrm{t})$

and

$$
-\frac{\partial}{\partial t} \eta_{j}^{*}(x, t)=\lambda_{j} F \chi_{j}^{T} \phi^{*}(x, t)-\lambda_{j} \eta_{j}^{*}(x, t)
$$

where the asterisks indicate adjoint functions, and the superscript $T$ signifies transposes of the respective matrices. When the eigenvalue problem for the omega-d modes is formed, and a corresponding eigenvalue problem is formed for the adjoint equations, the eigenfunctions for the flux problem are orthogonal to the eigenfunctions of the adjoint problem. ${ }^{53}$ The flux and adjoint eigenfunctions are then said to form a biorthogonal set. ${ }^{53}$ According to Becker, ${ }^{39}$ a stationary functional can always be written in terms of a system and its adjoint system. Accordingly, the following functional ${ }^{18}$ is a stationary functional for the system being considered in this chapter:

$$
\begin{aligned}
\mathrm{J} & =\int_{\mathrm{t}=0}^{\mathrm{t}_{\mathrm{f}}} \int_{\mathbf{x}}\left\{\nabla \phi^{* T} \cdot \mathrm{D} \nabla \phi-\phi^{* \mathrm{~T}}\left[\mathrm{~A}-(1-\beta) \chi_{\mathrm{p}} \mathrm{F}^{\mathrm{T}}\right] \phi-\frac{1}{2}\left(\phi^{*} \mathrm{~T}_{\mathrm{V}}^{-1} \frac{\partial}{\partial \mathrm{t}} \phi-\phi^{\mathrm{T}} \mathrm{V}^{-1} \frac{\partial}{\partial \mathrm{t}} \phi^{*}\right)\right. \\
& \left.+\sum_{\mathrm{j}=1}^{\mathrm{J}}\left[\phi^{* \mathrm{~T}} \lambda_{\mathrm{j}} \chi_{\mathrm{j}} \eta_{\mathrm{j}}+\eta_{\mathrm{j}}^{*} \beta_{\mathrm{j}} \mathrm{F}^{\mathrm{T}} \phi-\eta_{\mathrm{j}}^{*} \lambda_{\mathrm{j}} \eta_{\mathrm{j}}-\frac{1}{2}\left(\eta_{\mathrm{j}}^{*} \frac{\partial}{\partial \mathrm{t}} \eta_{\mathrm{j}}-\eta_{\mathrm{j}} \frac{\partial}{\partial \mathrm{t}} \eta_{\mathrm{j}}^{*}\right)\right]\right\} \mathrm{dxdt} .
\end{aligned}
$$


For simplicity, the initial conditions, final conditions, and boundary conditions have been omitted from the above. By taking arbitrary variations of $\mathrm{J}$ with respect to $\phi^{*}$, and allowing them to vanish, we obtain the groupdiffusion Eqs. 3.1 and 3.2. Similarly, by taking variations with respect to $\phi$; and allowing $\delta \mathrm{J}$ to vanish, we obtain the adjoint-system Eqs. 3.43 and 3.44 .

The functional $\mathrm{J}$ is stationary with respect to arbitrary variations in: $\phi^{*}$ and $\phi$, so that the value of $\mathrm{J}$ can be determined very accurately. In this case, though, since $J$ does not represent any physical quantity, the . advantage gained from using the variational principle is not clear.

As mentioned in Chapter II, when a variational method is used, the adjoint function must be expanded as a sum of trial functions; that is,

$$
\phi^{*}(\mathrm{x}, \mathrm{t})=\sum_{i=1}^{\mathrm{I}} \psi_{i}^{*}(\mathrm{x}) \mathrm{N}_{\mathrm{i}}^{*}(\mathrm{t})
$$

In addition, when Eqs. 3.3 and 3.46 are substituted into the variational principle, the taking of variations is essentially equivalent to using two complementary weighted-residual methods which determine the $N_{i}(t)$ and the $N_{i}^{*}(t)$. The trial functions for the first method are the weighting functions for the second, and vice versa,

If the choice

$$
\psi_{i}^{*}(\mathrm{x})=\psi_{\mathrm{i}}(\mathrm{x})
$$

is made, then the variational method is equivalent to using the Galerkin method to solve both the flux and adjoint problems. Indeed, even if Eq. 3.47 does not hold, there seems to be little advantage to using a variational method for solving space-time problems. Further, since the functional given by Eq. 3.45 can only be written for linear systems, a weightedresidual procedure should be adopted from the outset. There is no need to search for variational principles for solving space-time problems when the more simple weighted-residual formulation will do just as well.

It is possible, however, to find variational principles for nonlinear systems. Luco ${ }^{54}$ has formulated one for a two-energy group model with no delayed neutrons, using the following "constant power removal" model for the temperature change:

$$
\frac{\partial \mathrm{T}}{\partial \mathrm{t}}=\frac{\mathrm{K}}{\rho \mathrm{C}_{\mathrm{p}}}\left(\Sigma_{\mathrm{f}_{1} \phi_{1}}+\Sigma_{\mathrm{f}_{2} \phi_{2}}\right),
$$

where " $\mathrm{K}$ is a conversion factor from fissions to some unit of energy, $\rho$ is the density of the reactor material, and $C_{p}$ is its specific heat. "54 
When Eq. 3.48 is used with Eqs. 3.1 and 3.2 to formulate a variational principle, the concept of an adjoint temperature must be introduced. A set of adjoint equations is then derived from the variational principle by taking variations with respect to $\phi_{1}, \phi_{2}$, and $\mathrm{T}$. These equations are more complicated than Eqs. 3.43 and 3.44. Furthermore, the equation adjoint to Eq. 3.48 is so complicated that the real fluxes themselves appear in it. Nevertheless, the adjoint system is linear. ${ }^{39}$

Luco's derivation underscores the earlier observation that weightedresidual methods are preferable, in that it serves to illustrate the complexity of variational methods for even the most simple nonlinear systems. A1though the formulations are possible, they seem to be far less practical than the direct use of weighted-residual methods. 


\section{CHAPTER IV}

\section{SPATIALLY DISCONTINUOUS TRIAL FUNCTIONS}

\section{A. Spatial Discontinuities}

The spatially discontinuous trial-function method attempts to gain more flexibility by using trial functions suitable to local regions within a reactor, rather than to the entire reactor. The time dependence is then described by a different set of amplitude functions for each region. If trial functions are chosen properly, fewer are required within a region to obtain an accurate solution as compared with trial functions that vary continuously throughout the reactor. In addition, the numerical problem of "almost linear dependence" is easier to solve than in the continuous case, because only trial functions within a region need be compared. Linear dependence is discussed in Appendix A.

The discontinuities appear at the region interfaces. They fall into two classes, which can most easily be described with the aid of the two-

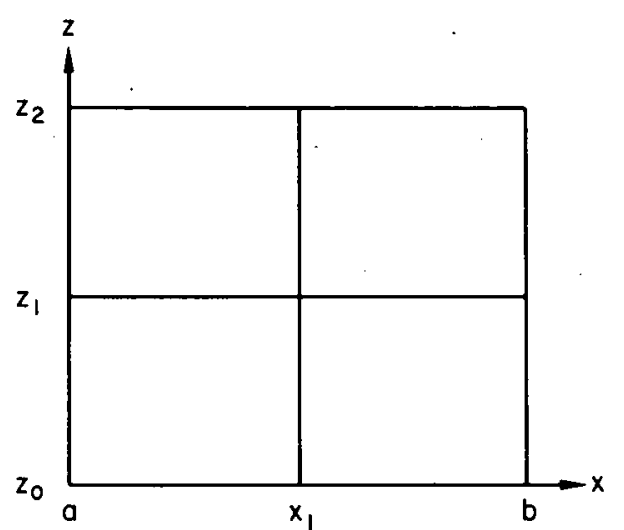

$113-1895$

Fig. 1. A Two-dimensional Reactor Illustrating Spatially Discontinuous Trial Functions dimensional "reactor" of Fig. 1. For this reactor, the trial functions are functions of the variable $x$, so that the undetermined functions will depend upon $z$ and $t$. In the first class, ${ }^{28}$ one set of functions continuous in $x$ is used between $z=0$ and $z=z_{1}$, and another set, also continuous in $x$, is used between $z_{1}$ and $z_{2}$. Since only one space dimension is being treated in this report the first class will not be discussed in this chapter. However; as will be seen in Chapter $\mathrm{V}$, the temporally discontinuous trial-function methods do fall into this class.

In the second class, known as multichannel synthesis, ${ }^{27,55}$ one set of trial functions is used for all $z$ and $t$. These trial

functions may exhibit discontinuities at $x_{1}$. The main feature of multichannel synthesis, however, is not that the trial functions are discontinuous, but that the regions ( 0 to $x_{1}, x_{1}$ to $b$ ) are each described by different sets of modes. Since neutrons born in one region can undergo an event in the other, interregion leakage must be considered. The usual way is to introduce coupling coefficients, which are multipliers of the fluxes and currents at the interfaces, for example, at $x_{1}$. In this way, an effort is made to preserve the diffusion-theory interface conditions of flux and current continuity.

The coupling-coefficient approach can only provide crude estimates of the interregion flux contributions. The coupling coefficients cannot 
account for changes in the flux shape and can only be time-dependent if the matrix of diffusion coefficients. is time-dependent. Nevertheless, the method has been. used successfully. ${ }^{55}$

B. The Role of Interface Residuals in Multichannel Synthes is

In this section, the multichannel-synthesis version of the multimode kinetics equations will be derived. Particular emphasis will be placed.on weighting the diffusion-theory. interface conditions. in order to derive the coupling coefficients.

Suppose a reactor is partitioned into $K$ synthesis regions, or channels, with interfaces at $x_{1}, \ldots, x_{K-1}$. Then, Eqs. 3.1, 3.2, 3.11, and 3.12 can be rewritten. in terms of the familiar multiregion formulation ${ }^{53}$ as follows (assuming one energy group for simplicity):

$$
\begin{aligned}
\frac{1}{v} \frac{\partial \phi_{k}}{\partial t}= & {\left[\nabla \cdot D_{k}(x, t) \nabla-\dot{A}(x, t)+(1-\beta) \chi_{p} F^{T}(x, t)\right] \phi_{k}(x, t) } \\
& +\sum_{j=1}^{J} \lambda_{j} \chi_{j} \eta_{j k}(x, t), \quad k=1, \ldots, k,
\end{aligned}
$$

$$
\begin{gathered}
\frac{\partial \eta_{j k}}{\partial t}=\beta_{j} F^{T}(x, t) \phi_{k}-\lambda_{j} \eta_{j k}(x, t), \quad k=1, \ldots, k, \quad j=1, \ldots, J, \\
\phi_{k}(x, 0)=\phi_{0 k}(x), \quad k=1, \ldots, k,
\end{gathered}
$$

and

$$
\phi_{1}(\mathrm{a}, \mathrm{t})=\phi_{\mathrm{K}}(\mathrm{b}, \mathrm{t})=0
$$

where the subscript $\mathrm{k}$ : indicates the $\mathrm{kth}$ channel. In addition to Eqs. 4.1-4.4, the system includes the following interface conditions, which preserve continuity of flux and current:

$$
\alpha_{1} \phi_{k}\left(x_{k}, t\right)=\alpha_{1} \phi_{k+1}\left(x_{k}, t\right), \quad k=1, \ldots, K-1
$$

and

$\left.\alpha_{2} D_{k}\left(x_{k}, t\right) \frac{\partial \phi_{k}}{\partial x}\right|_{\substack{x=x_{k} \\ \vdots}}=\left.\alpha_{2} D_{k+1}\left(x_{k}, t\right) \frac{\partial \phi_{k}}{\partial x}\right|_{x=x_{k}}, \quad k=1, \ldots, K-1$

The parameters $\alpha_{1}$ and $\alpha_{2}$ are, for now, unspecified: 
The synthesis approximation is made for each channel by expanding each $\phi_{\mathrm{k}}$ in the trial solutions:

$$
\bar{\phi}_{k}(x, t)=\sum_{i=1}^{I_{k}} \psi_{k i}(x) N_{k i}(t), \quad k=1, \ldots, K
$$

The integer $I_{k}$ is the number of trial functions chosen for the kth synthesis region. When the trial solutions are substituted into the system given by Eqs. 4.1-4.6, the following residuals are formed:

$$
\begin{aligned}
R_{F_{k}}(x, t)= & \frac{1}{v} \frac{\partial \bar{\phi}_{k}}{\partial t}-\left[\nabla \cdot D_{k}(x, t) \nabla-A(x, t)+(1-\beta) \chi_{p} F^{T}(x, t)\right] \bar{\phi}_{k}(x, t) \\
& -\sum_{j=1}^{J} \lambda_{j} \chi_{j} \eta_{j k}(x, t), \quad k=1, \ldots, K
\end{aligned}
$$

$\mathrm{R}_{\mathrm{P}_{\mathrm{jk}}}(\mathrm{x}, \mathrm{t})=\frac{\partial \eta_{\mathrm{jk}}}{\partial \mathrm{t}}-\beta_{\mathrm{j}} \mathrm{F}^{\mathrm{T}}(\mathrm{x}, \mathrm{t}) \bar{\phi}_{\mathrm{k}}+\lambda_{\mathrm{j}} \eta_{\mathrm{jk}}(\mathrm{x}, \mathrm{t}), \quad \mathrm{k}=1, \ldots, \mathrm{K}, \quad \mathrm{j}=1, \ldots, \mathrm{J}$,

$$
\begin{aligned}
& \mathrm{R}_{A}(t)=\bar{\phi}_{1}(\mathrm{a}, \mathrm{t}), \\
& \mathrm{R}_{\mathrm{B}}(\mathrm{t})=\bar{\phi}_{\mathrm{K}}(\mathrm{b}, \mathrm{t}), \\
& \mathrm{R}_{0 \mathrm{k}}(\mathrm{x})=\phi_{0 \mathrm{k}}(\mathrm{x})-\bar{\phi}_{\mathrm{k}}(\mathrm{x}, 0), \quad \mathrm{k}=1, \ldots, \mathrm{K}, \\
& \mathrm{R}_{I_{\cdot k}}\left(\mathrm{x}_{\mathrm{k}}, \mathrm{t}\right)=\alpha_{1} \bar{\phi}_{\mathrm{k}+1}\left(\mathrm{x}_{\mathrm{k}}, \mathrm{t}\right)-\alpha_{1} \bar{\phi}_{\mathrm{k}}\left(\mathrm{x}_{\mathrm{k}}, \mathrm{t}\right), \quad \mathrm{k}=1, \ldots, \mathrm{K}-1,
\end{aligned}
$$

and

$$
\begin{aligned}
R_{J_{k}}\left(x_{k}, t\right)= & \left.\alpha_{2} D_{k+1}\left(x_{k}, t\right) \frac{\partial \bar{\phi}_{k+1}}{\partial x}\right|_{x=x_{k}} \\
& -\left.\alpha_{2} D_{k}\left(x_{k}, t\right) \frac{\partial \bar{\phi}_{k}}{\partial x}\right|_{x=x_{k}}, \quad k=1, \ldots, \dot{k}-1
\end{aligned}
$$

Equations 4.8-4:12 are the multiregion equivalents of Eqs. 3.9, 3.10, $3.13,3.14$, and 3.15. Equations 4.13 and 4.14 are called the interface residuals. They are not unique, however. Adding Eq. 4.6 to Eq. 4.5 and subtracting Eq. 4.6 from Eq. 4.5 produce the following equivalent interface conditions: 


$$
\begin{gathered}
\alpha_{1} \phi_{k}\left(x_{k}, t\right)+\left.\alpha_{2} D_{k}\left(x_{k}, t\right) \frac{\partial \phi_{k}}{\partial x}\right|_{x=x_{k}}:=\alpha_{1} \phi_{k+1}\left(x_{k}, t\right)+\left.\alpha_{2} D_{k+1}\left(x_{k}, t\right) \frac{\partial \phi_{k+1}}{\partial x}\right|_{x=x_{k}}, \\
k=1, \ldots, K-1
\end{gathered}
$$

and

$$
\begin{aligned}
& \alpha_{1} \phi_{k}\left(x_{k}, t\right)-\left.\alpha_{2} D_{k}\left(x_{k}, t\right) \frac{\partial \phi_{k}}{\partial x}\right|_{x=x_{k}}=\alpha_{1} \phi_{k+1}\left(x_{k}, t\right)-\left.\alpha_{2} D_{k+1}\left(x_{k}, t\right) \frac{\partial \phi_{k+1}}{\partial x}\right|_{x=x_{k}} \\
& k=1, \ldots, k-1
\end{aligned}
$$

When Eq. 4.7 is substituted into Eqs. 4.15 and 4.16 , the following residuals are formed:

$$
\begin{aligned}
\mathrm{R}_{\mathrm{S}_{\mathrm{k}}}\left(\mathrm{x}_{\mathrm{k}}, \mathrm{t}\right)= & \alpha_{1} \bar{\phi}_{\mathrm{k}+1}\left(\mathrm{x}_{\mathrm{k}}, \mathrm{t}\right)-\alpha_{1} \bar{\phi}_{\mathrm{k}}\left(\mathrm{x}_{\mathrm{k}}, \mathrm{t}\right)+\left.\alpha_{2} \mathrm{D}_{\mathrm{k}+1}\left(\mathrm{x}_{\mathrm{k}}, \mathrm{t}\right) \frac{\partial \bar{\phi}_{\mathrm{k}+1}}{\partial \mathrm{x}}\right|_{\mathrm{x}=\mathrm{x}_{\mathrm{k}}} \vdots \\
& -\left.\alpha_{2} D_{\mathrm{k}}\left(\mathrm{x}_{\mathrm{k}}, \mathrm{t}\right) \frac{\partial \bar{\phi}_{\mathrm{k}}}{\partial \mathrm{x}}\right|_{\mathrm{x}=\mathrm{x}_{\mathrm{k}}}, \quad \mathrm{k}=1, \ldots, \mathrm{K}-1,
\end{aligned}
$$

and

$$
\begin{aligned}
\mathrm{R}_{\mathrm{Dk}_{\mathrm{k}}}\left(\mathrm{x}_{\mathrm{k}}, \mathrm{t}\right)= & \alpha_{1} \bar{\phi}_{\mathrm{k}+1}\left(\mathrm{x}_{\mathrm{k}}, \mathrm{t}\right)-\alpha_{1} \bar{\phi}_{\mathrm{k}}\left(\mathrm{x}_{\mathrm{k}}, \mathrm{t}\right)-\left.\alpha_{2} \mathrm{D}_{\mathrm{k}+1}\left(\mathrm{x}_{\mathrm{k}}, \mathrm{t}\right) \frac{\partial \bar{\phi}_{\mathrm{k}+1}}{\partial \mathrm{x}}\right|_{\mathrm{x}=\mathrm{x}_{\mathrm{k}}} \\
& +\left.\alpha_{2} \mathrm{D}_{\mathrm{k}}\left(\mathrm{x}_{\mathrm{k}}, \mathrm{t}\right) \frac{\partial \bar{\phi}_{\mathrm{k}}}{\partial \mathrm{x}}\right|_{\mathrm{x}=\mathrm{x}_{\mathrm{k}}}, \quad \mathrm{k}=1, \ldots, \mathrm{K}-1 .
\end{aligned}
$$

The residuals given by Eqs. 4.17 and 4.18. differ from those given by Eqs. 4.13 and 4.14. Nevertheless, either set is permissible.

The residuals can be weighted and the weighted-residual method used. However, further explanation is needed. In Chapter II, it was mentioned that surface integrals.should be formed.when using the weightedresidual method on the boundary.conditions. The boundary weighted.residuals should then either be added to or subtracted from the differentialequation. weighted residuals in order to be able to maintain a completely determinable system. The sum (or difference) should then be allowed to vanish. In this chapter, interface residuals are treated in the same manner。 
In theory, the weighting. functions for the interface residuals are arbitrary. In practice, though, they are usually closely related to the weighting functions used to weight Eqs. 4.8 and 4.9. Stacey's variational method, ${ }^{55}$ for example, would weight Eqs. 4.13 and 4.14 with the following:

$W_{I_{k, i_{k}}}\left(x_{k}, t\right)=\frac{1}{2}\left[\left.D_{k}\left(x_{k}, t\right) \frac{d W_{k, i_{k}}}{d x}\right|_{x=x_{k}}+\left.D_{k+1}\left(x_{k}, t\right) \frac{d W_{k+1, i_{k+1}}}{d x}\right|_{x=x_{k}}\right]$,

and

$$
\begin{gathered}
W_{J_{k, i_{k}}}\left(x_{k}\right)=\frac{1}{2}\left[w_{k, i_{k}}\left(x_{k}\right)+w_{k+1}, i_{k+1}\left(x_{k}\right)\right], \quad i_{k}=1, \ldots, I_{k} ; \\
i_{k+1}=1, \ldots, I_{k+1} ; \quad j=1, \ldots, K-1 .
\end{gathered}
$$

The factor $1 / 2$, however, automatically implies that each channel contains an equal number of trial functions; that is, $I_{k}=I_{k+1}$. A more general approach ${ }^{56,57}$ would be to chose

$$
\begin{aligned}
W_{I_{k, i_{k}}}\left(x_{k}, t\right)= & \left.\gamma D_{k}\left(x_{k}, t\right) \frac{d W_{k, i_{k}}}{d x}\right|_{x=x_{k}} \\
& +\left.(1-\gamma) D_{k+1}\left(x_{k}, t\right) \frac{d W_{k+1, i_{k+1}}}{d x}\right|_{x=x_{k}},
\end{aligned}
$$

and

$$
\begin{gathered}
W_{J_{k, i_{k}}}\left(x_{k}\right)=(1-\gamma) w_{k, i_{k}}\left(x_{k}\right)+\gamma W_{k+1, i_{k+1}}\left(x_{k}\right), \quad i_{k}=1, \ldots, I_{k} ; \\
\vdots \\
i_{k+1}=1, \ldots, I_{k+1} ; \quad k=1, \ldots, K-1,
\end{gathered}
$$

where $0 \leq \gamma \leq 1$. When $\gamma$ is either zero or one, each region may have different numbers of trial functions. ${ }^{57}$ If zero is chosen, the flux-interface conditions are weighted by the $I_{k+1}$ values of the derivative of the weighting functions of the region to the right of the interface. The current-interface conditions are weighted by the $I_{k}$ values of the weighting functions of the region to the left of the interface. If $\gamma$ is chosen to be unity, the fluxinterface conditions are weighted by the $I_{k}$ values of the derivatives of the left-side weighting functions while the current-interface residuals are weighted by the $I_{k+1}$ values of the right-side weighting functions. 
The choices $\gamma=0$.or $\gamma=1$ appear to be equally valid. Further more, if each region contains the same number of modes, no value of $\gamma$ appears to deserve preference. Pomraning, ${ }^{56}$ however, in his discussion of temporally discontinuous trial functions, concludes that $\gamma=1 / 2$ is the most "natural" choice. Pomraning's arguments are discussed further in. Chapter V.

The argument can be repeated for the weighting of Eqs. 4.17 and 4.18. This time, however,

$$
W_{S_{k, i}}\left(x_{k}\right)=\gamma W_{k, i_{k}}\left(x_{k}\right)+(1-\gamma) W_{k+1, i_{k+1}}\left(x_{k}\right)
$$

and

$$
\begin{aligned}
& W_{D_{k, i_{k}}}\left(x_{k}\right)=(1-\gamma) W_{k, i_{k}}\left(x_{k}\right)+\gamma W_{k+1, i_{k+1}}\left(x_{k}\right), \quad i_{k}=1, \ldots, I_{k} \\
& i_{k+1}=1, \ldots, I_{k+1}, \quad k=1, \ldots, K-1
\end{aligned}
$$

Equations 4.23 and 4.24, with $\gamma=1$, will now be used to complete the derivation of the multimode kinetics equations of multichannel synthesis.

The weighted-residual procedure is now performed by multiplying Eq. 4.8 by the weighting functions $W_{k, i}(x)$, integrating over region $k$, and subtracting the interface weighted residuals obtained by multiplying Eq. 4.17. by Eq. 4.23, and Eq. 4.1 .8 by Eq. 4. 25:

$$
\begin{aligned}
0 .= & \int_{x_{k-1}}^{x_{k}} \cdot W_{k, i_{k}}(x) R_{F_{k}}(x, t) d x-W_{k, i_{k}} \cdot\left(x_{k}\right) R_{S_{k}}\left(x_{k}, t\right) \\
& -W_{k, i_{k}}\left(x_{k-1}\right) R_{D_{k-1}}\left(x_{k-1}, t\right), \quad k=1, \ldots, K,
\end{aligned}
$$

where

$$
x_{0}=a \text {, and } x_{K}=b \text {. }
$$

Weighted-residual criteria for the precursor equations and the initial conditions result when the weighting. functions. $W_{k, i_{k}}(x)$ multiply Eqs. 4.9 . and 4.12 :

$$
\int_{x_{k-1}}^{x_{k}} w_{k, i_{k}}(x) R_{P_{j k}}(x, t) d x=0
$$


and

$\int_{x_{k-1}}^{x_{k}} W_{k, i_{k}}(x) R_{0 k}(x) d x=0, \quad i_{k}=1, \ldots, I_{k}, \ldots, k=1, \ldots, K$

where

$$
\mathbf{x}_{0}=\mathrm{a} \text {, and } \mathrm{x}_{\mathrm{K}}=\mathrm{b}
$$

The weighted-residual criteria given by Eqs. 4.25-4.27 are equivalent to the multimode kinetics equations. Therefore, a reactor divided into $\mathrm{K}$. synthesis regions with $\mathrm{I}_{\mathrm{k}}$ modes in each region could be described by Eqs. 3.32 and 3.33, with the coefficients of the matrices $\rho, \bar{\beta}_{j}$, and $\Lambda$ described às follows:

$\Lambda$ is a block diagonal ${ }^{58}$ I x I matrix

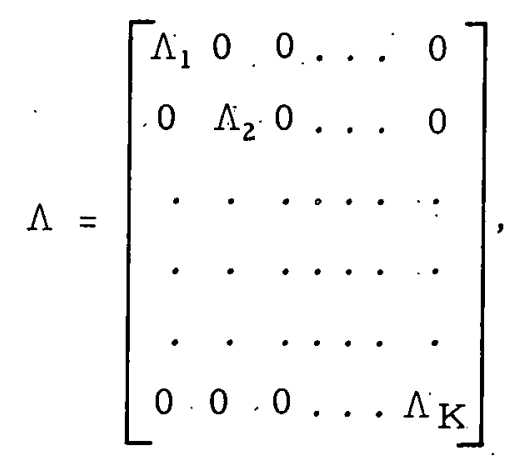

where each $\Lambda_{k}$ is a square submatrix of order $I_{k}$. Note that

$$
I .=\sum_{k=1}^{K} I_{k}
$$

Within a submatrix $\Lambda_{\mathrm{k}}$, an element is

$\Lambda_{\mathrm{mn}}^{(\mathrm{k})}=\int_{\mathrm{x}_{\mathrm{k}-1}}^{\mathrm{x}_{\mathrm{k}}} \cdot \mathrm{W}_{\mathrm{k}, \mathrm{m}}(\mathrm{x}) \frac{\mathrm{l}}{\mathrm{v}} \psi_{\mathrm{k}, \mathrm{n}}(\mathrm{x}) \mathrm{dx}, \quad \mathrm{m}=1, \ldots, \mathrm{I}_{\mathrm{k}}, \quad \mathrm{n}=1, \ldots, \mathrm{I}_{\mathrm{k}}$

The $\bar{\beta}_{\mathrm{j}}$ matrices are also block diagonal matrices 


$$
\bar{\beta}_{\mathrm{j}}(\mathrm{t})=\left[\begin{array}{ccccc}
\bar{\beta}_{\mathrm{j} 1} & 0 & 0 & \ldots & 0 \\
0 & \bar{\beta}_{\mathrm{j} 2} & 0 & \ldots & 0 \\
\cdot & \cdot & \ldots & \vdots \\
\cdot & \cdot & \ldots & \cdot \\
\cdot & \cdots & \ldots & \cdot \\
0 & 0 & 0 & \ldots & \bar{\beta}_{\mathrm{jK}}
\end{array}\right], \quad \mathrm{j}=1, \ldots, \mathrm{J}
$$

Within a submatrix $\bar{\beta}_{j k}$, the elements are

$$
\begin{gathered}
\bar{\beta}_{\mathrm{jmn}}^{(\mathrm{k})}(\mathrm{t})=\beta_{\mathrm{j}} \int_{\mathrm{x}_{\mathrm{k}-1}}^{\mathrm{x}_{\mathrm{k}}} \mathrm{W}_{\mathrm{k}, \mathrm{m}}(\mathrm{x}) \chi_{\mathrm{j}} \mathrm{F}^{\mathrm{T}}(\mathrm{x}, \mathrm{t}) \psi_{\mathrm{k}, \mathrm{n}}(\mathrm{x}) \mathrm{dx}, \quad \mathrm{m}=1, \ldots, \mathrm{I}_{\mathrm{k}} \\
\mathrm{n}=1, \ldots, \mathrm{I}_{\mathrm{k}} .
\end{gathered}
$$

Finally, $\rho(t)$ is an I $x$ I block tridiagonal matrix ${ }^{58}$ given by

$$
\rho(t)=\left[\begin{array}{ccccccccc}
\rho_{1}^{(1)} & \rho_{1}^{(2)} & 0 & 0 & . & \cdot & \cdot & 0 & 0 \\
\rho_{2}^{(1)} & \rho_{2}^{(2)} & 0 & 0 & \cdot & \cdot & \cdot & 0 & 0 \\
\cdot & \cdot & \cdot & \cdot & \cdot & \cdot & \cdot & \cdot & \cdot \\
\cdot & \cdot & \cdot & \cdot & \cdot & \cdot & \cdot & \cdot & \ddots \\
\cdot & \cdot & \cdot & \cdot & \cdot & \cdot & \cdot & \cdot & \cdot \\
0 & 0 & 0 & 0 & \cdot & \cdot & \cdot & \rho_{\mathrm{K}-1}^{(\mathrm{K}-1)} & \rho_{\mathrm{K}-1}^{(\mathrm{K})} \\
0 & 0 & 0 & 0 & \cdot & \cdot & \cdot & \rho_{\mathrm{K}}^{(\mathrm{K}-1)} & \rho_{\mathrm{K}}^{(\mathrm{K})}
\end{array}\right] .
$$

The submatrices $\rho_{\mathrm{k}}^{(\mathrm{k})}(\mathrm{t})$ on the main diagonal contain elements given by 


$$
\begin{aligned}
\rho_{m n}^{(k)}(t)= & \int_{x_{k-1}}^{x_{k}}\left\{W_{k, m}(x)\left[(1-\beta) \chi_{p} F^{T}(x, t)-A(x, t)\right] \psi_{k, n}(x)-D_{k}(x, t) \frac{d W_{k, m}}{d x} \frac{d \psi_{k, n}}{d x}\right\} d x \\
& -W_{k, m}\left(x_{k-1}\right)\left[\alpha_{1} \psi_{k, n}\left(x_{k-1}\right)+\left.\left(1-\alpha_{2}\right) D_{k}\left(x_{k-1}, t\right) \frac{d \psi_{k, n}}{d x}\right|_{x=x_{k-1}}\right] \\
& -W_{k, m}\left(x_{k}\right)\left[\alpha_{1} \psi_{k, n}\left(x_{k}\right)-\left.\left(1-\alpha_{2}\right) D_{k}\left(x_{k}, t\right) \frac{d \psi_{k, n}}{d x}\right|_{x=x_{k}}\right] \\
& m=1, \ldots, I_{k}, \quad n=1, \ldots, I_{k} .
\end{aligned}
$$

These elements account for the intraregion processes. The interregion processes are represented by the coupling coefficients, which are discussed next.

The submatrices $\rho_{k-1}^{(k)}(t)$ on the supradiagonal represent the coupling from the region to the right of interface $k$ to the region to the left of the interface. The matrix elements for this "backward" coupling are given by

$$
\begin{aligned}
\rho_{\mathrm{mn}}^{(\mathrm{k})}(\mathrm{t}) & =\mathrm{W}_{\mathrm{k}, \mathrm{m}}\left(\mathrm{x}_{\mathrm{k}}\right)\left[\alpha_{1} \psi_{\mathrm{k}+1, \mathrm{n}}\left(\mathrm{x}_{\mathrm{k}}\right)+\left.\alpha_{2} \mathrm{D}_{\mathrm{k}+1}\left(\mathrm{x}_{\mathrm{k}}, \mathrm{t}\right) \frac{\mathrm{d} \psi_{\mathrm{k}+1, \mathrm{n}}}{\mathrm{dx}}\right|_{\mathrm{x}=\mathrm{x}_{\mathrm{k}}}\right] \\
\mathrm{m} & =1, \ldots, \mathrm{I}_{\mathrm{k}}, \quad \mathrm{n}=1, \ldots, \mathrm{I}_{\mathrm{k}+1} .
\end{aligned}
$$

The submatrices $\rho_{k}^{(k-1)}(t)$ on the subdiagonal represent the coupling from the region to the left of interface $k$ to the region to the right of the interface. The matrix elements for this "forward" coupling are

$$
\begin{aligned}
\rho_{\mathrm{mn}}^{(\mathrm{k})}(\mathrm{t}) & =\mathrm{W}_{\mathrm{k}+1, \mathrm{~m}}\left(\mathrm{x}_{\mathrm{k}}\right)\left[\alpha_{1} \psi_{\mathrm{k}, \mathrm{n}}\left(\mathrm{x}_{\mathrm{k}}\right)-\left.\alpha_{\mathrm{z}} \mathrm{D}_{\mathrm{k}}\left(\mathrm{x}_{\mathrm{k}}, \mathrm{t}\right) \frac{\mathrm{d} \psi_{\mathrm{k}, \mathrm{n}}}{\mathrm{dx}}\right|_{\mathrm{x}=\mathrm{x}_{\mathrm{k}}}\right], \\
\mathrm{m} & =1, \ldots, \mathrm{I}_{\mathrm{k}+1}, \quad \mathrm{n}=1, \ldots, \mathrm{I}_{\mathrm{k}} .
\end{aligned}
$$

The usual matrix notation can be obtained for the elements of $\Lambda, \rho$, and $\bar{\beta}_{j}$ by expanding the submatrices within the large matrix and relabeling the matrix elements.

The amplitude functions and the precursor amplitude functions also must be relabeled. Within the kth synthesis region, the precursor amplitude functions are 


$$
\begin{gathered}
C_{j k m}(t)=\int_{x_{k-1}}^{x_{k}} W_{k m}(x) \chi_{j} \eta_{j k}(x, t) d x, \quad m=1, \ldots, I_{k}, \\
j=1, \ldots, \text { J. . }
\end{gathered}
$$

To effect the relabeling, the amplitude functions $\mathrm{N}_{\mathrm{km}}(\mathrm{t})$ (see Eq. 4.7) and the precursor amplitude functions from each region are placedin Idimensional column vectors from the top, beginning. with $\mathrm{k}:=1$, and proceeding channel by channel, until the vectors are filled.

Equations 4.33-4.35 contain the unspecified parameters $\alpha_{1}$ and $\alpha_{.2}$, which were introduced as multipliers of the interface conditions, Eqs. 4.5 and 4.6. When $\alpha_{1}$ and $\alpha_{2}$ are both unity, there are no interface current contributions to Eq. 4.33. This choice shall be called surface-cancellation weighting. If $\alpha_{1}=1 / 4$ and $\alpha_{2}=1 / 2$, however, the surface terms in each equation are the partial currents at the interfaces. Consequently, this choice shall be called partial-current weighting. These weighting techniques are compared in Chapter VIII.

Unfortunately, the coupling coefficients are time-dependent only. when the diffusion coefficients are time-dependent. The formulation described above assumes time-independent shape functions. However, the contribution to the total flux in one region from the changing flux in the others is not neglected, because the coupling coefficients multiply the amplitude functions.

Another limitation of the method is a direct consequence of the diffusion-theory interface conditions: :nonadjacent region coupling must be neglected. This assumption is very poor when the synthesis regions are small.

\section{A Nodal Approximation}

If only one trial function is chosen in each synthesis region, the multichannel-synthesis method reduces to a nodal method. ${ }^{16,59}$ Many, nodal methods have been formulated from many different points of view. The multichannel-synthesis version is one of the few that are consistent. It lies completely within. the confines of diffusion theory. Consequently, it should not be expected to do as well as those based on transport theory. ${ }^{60,61}$ Another drawback is that shape changes with time do not appear in the definitions of the shape functions.

When $\alpha_{1}=1 / 4$ and $\alpha_{2}=1 / 2$, the coupling coefficients are proportional to the partial currents at the interfaces. This choice has been assigned.to a nodal approximation in an ad.hoc derivation by Kaplan. ${ }^{62}$. There does not seem to be any a priori reason.why this choice of $\alpha_{1}$ and $\alpha_{2}$ should be better or worse than any other choice. Accordingly, Chapter. VIII contains a numerical comparison of partial-current weighting and surface-cancellation weighting in the nodal approximation derived above. 
CHAPTER V

TEMPORALLY.DISCONTINUOUS TRIAL FUNCTIONS

A. Introduction

In a highly nonlinear transient, the flux shape can change drastically and continually, so that the "bracketing" idea discussed in Chapter III would in this case be essentially useless. Accordingly, several investigators ${ }^{14,15,29,55}$ have developed methods that provide means of changing the shape functions at selected times during a transient analysis. Some of these methods use precalculated shapes, ${ }^{29,55}$ and others recalculate the shapes in some manner during the analysis. ${ }^{14,15}$ The former are known as temporally discontinuous flux-synthesis methods; the latter are called quasistatic or factorization methods. Each can be regarded as a space-time weighted-residual method, as is shown in this chapter.

In Chapter IV, it was stated that the temporal discontinuities are similar to the first type of spatial discontinuities. To see this more clearly,

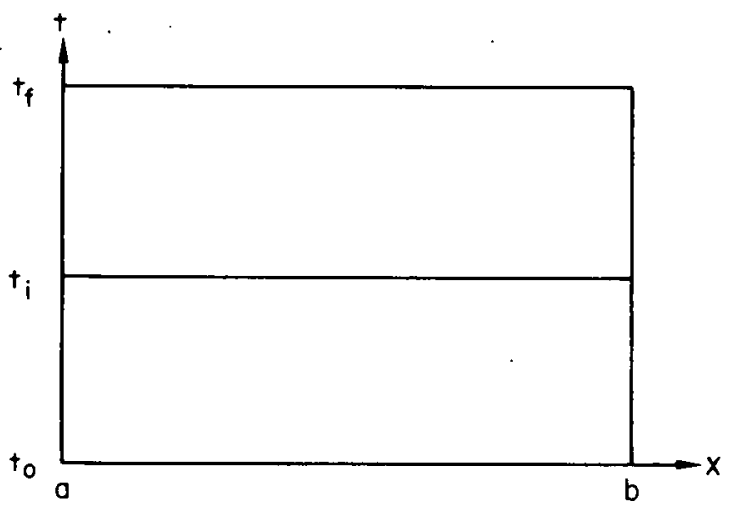

$113-1897$

Fig. 2. Illustration of the Use of Temporally Discontinuous Trial Functions consider Fig. 2, which shows a onedimensional reactor undergoing a transient. It is being analyzed with one set of shape functions from $t_{0}$ to $t_{i}$, and a second set from $t_{i}$ to $t_{f}$. Each set, however, is continuous in $x$. As will be seen, this type of discontinuity does not give rise to coupling coefficients. Instead, a new "initial residual" is required when the set of trial functions is changed.

Errors can be introduced when one set of trial functions is substituted for another, or, more realistically, if one trial function is removed from the set and another substituted for it. Consequently, jump discontinuities in the neutron flux and the precursor concentrations will result. If trial functions are removed, they should be "unimportant": in order that the sizes of the discontinuities be minimized, that is, if their corresponding amplitude functions are small. If the shape functions are recalculated at $t_{i}$, discontinuities can still result, unless some corrective action is taken. Such action usually takes the form of an iterative technique for shape-function recalculation. The newly calculated shapes and the previous shapes are used in linear combination for each iteration until the true shape function is found.

Section B below contains a weighted-residual,treatment of temporal discontinuities. The treatment extends the derivation of the multimode 
kinetics equations given in Chapter III to admit temporally discontinuous trial functions. Section $C$ of this chapter contains a discussion of the quasistatic (or factorization) method.

B. A Weighted-residual Treatment of Temporal Discontinuities

If shape functions are changed at $t=t_{i}$ during the analysis of a transient, an additional condition must be added to the system of equations given by Eqs. 3.1, 3.2, 3.11, and 3.12. This condition states that the true flux must not exhibit a jump discontinuity at $t=t_{i}$ :

$$
\phi\left(x, t_{i+}\right)=\phi\left(x, t_{i-}\right)
$$

For simplicity, a one-energy group model is adopted.in this chapter. In addition, the subscripts 1 and 2 denote the values of functions when $t_{0} \leq t<t_{i}$, and $t_{i}<t \leq t_{f}$, respectively. Thus, Eq. 5.1 can be rewritten as

$$
\phi_{1}\left(x, t_{i}\right)=\phi_{2}\left(x, t_{i}\right)
$$

Instead of the trial solution given by Eq. 3.3, consider the following trial solutions:

$$
\bar{\phi}_{1}(x, t)=\sum_{i=1}^{I_{1}} \psi_{i_{1} .}(x, t) N_{i_{1}}(t) \equiv \psi_{1}(x, t) N_{1}(t),
$$

and

$$
\bar{\phi}_{2}(x, t)=\sum_{i=1}^{I_{2}} \psi_{i 2}(x, t) N_{i 2}(t) \equiv \psi_{2}(x, t) N_{2}(t),
$$

where $I_{1}$ and $I_{2}$ are the number of trial functions chosen in the time intervals $t_{0} \leq t<t_{i}$ and $t_{i}<t \leq t_{f}$, respectively. The matrices $\psi_{1}$ and $\psi_{2}$ and the column vectors $\mathrm{N}_{1}$ and $\mathrm{N}_{2}$ are given by

$$
\begin{aligned}
& \psi_{1}(x, t)=\left[\psi_{11}(x, t) \ldots \psi_{I_{11}}(x, t)\right] \\
& \psi_{2}(x, t)=\left[\psi_{12}(x, t) \ldots \psi_{I_{22}}(x, t)\right] \\
& N_{1}(t)=\left[\begin{array}{c}
N_{11}(t) \\
\cdot \\
\cdot \\
\cdot \\
N_{I_{11}}(t)
\end{array}\right],
\end{aligned}
$$


and

$$
N_{2}(t)=\left[\begin{array}{c}
N_{12}(t) \\
\vdots \\
N_{I_{22}}(t)
\end{array}\right] .
$$

When Eqs. 5.3 and 5.4 are substituted into Eqs. 3.1, 3.2, 3.11, 3.12, and 5.2, the residuals $R_{F_{1}}(x, t), R_{F_{2}}(x, t), R_{P_{j 1}}(x, t), R_{P_{j}}(x, t), R_{I_{1}}(x), R_{I_{2}}(x), R_{A_{1}}(t)$, $R_{A_{2}}(t), R_{B_{1}}(t)$, and $R_{B_{2}}(t)$ are formed. These residuals are analogous to those given by Eqs. 3.9, 3.10, 3.13, 3.14, and 3.15; those subscripted.with 1 . are for the first time interval; those subscripted.with 2 are for the second time interval. The residual $R_{I_{2}}(x)$, then, can be interpreted as the initial residual for the second time interval and is written as

$$
R_{I_{2}}(x)=\overline{\phi_{2}}\left(x, t_{i}\right)-\overline{\phi_{1}}\left(x, t_{i}\right) \text {. }
$$

It is evident that the weighted-residual method used to derive the multimode kinetics equations in Chapter III can be repeated here to give two systems of multimode kinetics equations, one for each time interval. When the weighted-residual method is applied to Eq. 5.9, the initial conditions for the amplitude functions to be used in the second time interval will be obtained. They will be expressed in terms of the values at $t=t_{i}$ of the amplitude functions used for the first time interval. However, this use of Eq. 5.9. is not straightforward, as is now shown.

The following questions must be answered if temporally discontinuous trial functions are to be used: What if the weighting functions are changed at $t=t_{i}$ as well?. Which set should be used? What if $I_{1} \neq I_{2}$; that is, what should be done if the number of trial functions is changed?

Consider the weighting-function matrices

$$
\begin{aligned}
& W_{1}(x, t)=\left[W_{1.1}(x, t) \ldots W_{I_{11}}(x, t)\right] \\
& W_{2}(x, t)=\left[W_{12}(x, t) \ldots W_{I_{22}}(x, t)\right]
\end{aligned}
$$

and

$$
W_{i}(x)=\left[W_{1 i}(x) \ldots W_{I_{2}} i(x)\right]
$$

which weight, respectively, the residuals for the two time intervals, and the initial residual for the second time interval. Assuming that choices for $W_{1}$ and $W_{2}$ have already been made, the problem is to select $W_{i}(x)$. Some 
reasonable choices seem to be $W_{1}\left(x, t_{i}\right), W_{2}\left(x, t_{i}\right)$, or some linear combination of the two. A closer inspection of Eqs. 5.10-5.12 reveals, however, that since $I_{1}$ may not equal $I_{2}, W_{1}\left(x, t_{i}\right)$ cannot, in general, be chosen for $W_{i}(x)$, whereas $W_{2}\left(x, t_{i}\right)$ can always be chosen. This fact was first recognized by Becker. ${ }^{57}$

Suppose, on the other hand, that $I_{1}=I_{2}$. Then, $W_{i}(x)$ can be chosen as any linear combination of $W_{1}\left(x, t_{i}\right)$ and $W_{2}\left(x, t_{i}\right)$; for example,

$$
W_{i}(x)=\alpha W_{1}\left(x, t_{i}\right)+(1-\alpha) W_{2}\left(x, t_{i}\right) \text {, }
$$

where $0 \leq \alpha \leq 1$, but is otherwise arbitrary. Indeed, as Pomraning ${ }^{56}$ points out, $\alpha$ is so arbitrary that it can even be a function of $x$. He goes on to conclude, on "pseudophysical grounds, " grounds, ${ }^{63}$ that the most reasonable choice is $\alpha=1 / 2$. His mathematical argument will not be considered here. However, he argues on a "physical" basis that each weighting function is considered equally when $\alpha=1 / 2$. Even if he is correct, Becker's observation regarding unequal numbers of trial functions in each time interval, which would set $\alpha=0$, makes the argument somewhat irrelevant. The initial conditions for the amplitude functions used in the second time interval can now be found, with $\alpha=0$; that is,

$$
W_{i}(x)=W_{2}\left(x, t_{i}\right)
$$

When Eq. 5.9 is multiplied by the weighting function $(1 / v) W_{2}^{T}\left(x ; t_{j}\right)$, and the result integrated over all $x$, the result is

$$
\int_{a}^{b} w_{2}^{T}\left(x, t_{i}\right) \frac{1}{v}\left[\bar{\phi}_{2}\left(x, t_{i}\right)-\bar{\phi}_{1}\left(x, t_{i}\right)\right] d x=0 .
$$

If Eqs. 5.3 and 5.4 are used, Eq. 5.15 becomes

$$
\begin{aligned}
0= & \int_{a}^{b} w_{2}^{T}\left(x, t_{i}\right) \frac{1}{v} \psi_{2}\left(x, t_{i}\right) d x N_{2}\left(t_{i}\right) \\
& -\int_{a}^{b} w_{2}^{T}\left(x, t_{i}\right) \frac{1}{v} \psi_{1}\left(x, t_{i}\right) d x N_{1}\left(t_{i}\right) .
\end{aligned}
$$

The $I_{2} \times I_{2}$ matrix in the first term is immediately recognized as the generation time matrix $\Lambda_{2}\left(t_{i}\right)$. The $I_{2} \times I_{1}$ matrix in the second term shall be labeled $V_{21}\left(t_{i}\right)$ and is given by 


$$
v_{2 !}\left(t_{\dot{1}}\right)=\int_{a}^{b} w_{2}^{T}\left(x, t_{i}\right) \frac{l}{v} \psi_{1}\left(x, t_{i}\right) d x .
$$

Equation 5.16 becomes

$$
\Lambda_{2}\left(t_{i}\right) N_{2}\left(t_{i}\right)=V_{21}\left(t_{i}\right) N_{1}\left(t_{i}\right) .
$$

Finally, the initial conditions $\mathrm{N}_{2}\left(\mathrm{t}_{\dot{\mathrm{i}}}\right)$ are determined by

$$
N_{2}\left(t_{i}\right)=\Lambda_{2}^{-1}\left(t_{i}\right) V_{21}\left(t_{i}\right) N_{1}\left(t_{i}^{\prime}\right),
$$

and the multimode kinetics equations can now be solved over the second time interval.

Both quasistatic methods and flux-synthesis methods have been treated by the above analysis. The temporally discontinuous synthesis methods differ from the quasistatic methods.in the method of choosing the trial functions $\psi_{1}$ and $\psi_{2}$. These are precalculated.in a synthesis method to be used.beginning at $t=t_{0}$ and $t=t_{i}$, respectively. The quasistatic method recalculates the trial functions when $t=t_{i}$, using the trial functions $\psi_{1}\left(x, t_{0}\right)$ along. with an interative scheme. This: important method is described in detail in Section C below.

C. The Quasistatic Method

\section{Main.Features}

The quasistatic methods ${ }^{8,13-15}$ have always been formulated using only, one mode, that is, $I_{1}=I_{2}=1$, in Eqs. 5.3 and 5.4. In addition, the weighting functions have always been the steady-state adjoint functions. The multimode kinetics equations then reduce to the point-reactor model, which is used until a shape recalculation is to be made. To derive the equation. with which the second shape can be calculated, Eq. 3.3 is substituted into Eq: 3.1 to obtain

$$
\begin{aligned}
\frac{1}{v} \frac{\partial \psi}{\partial t}= & {\left[\nabla \cdot D \nabla-A+(1-\beta) \chi_{p} F^{T}-\frac{1}{v N(t)} \frac{d N}{d t}\right] \psi(x, t) } \\
& +\frac{1}{N(t)} \sum_{j=1}^{J} \lambda_{j} \chi_{j} \eta_{j}(x, t) .
\end{aligned}
$$

In addition, the constraint condition, Eq. 3.23 becomes 


$$
\frac{\mathrm{d}}{\mathrm{dt}} \int_{\mathrm{x}} \mathrm{W}^{\mathrm{T}}(\mathrm{x}) \frac{1}{\mathrm{v}} \psi(\mathrm{x}, \mathrm{t}) \mathrm{dx}=0
$$

The shape equation is coupled to the amplitude equation in three ways:

a. The precursor term in Eq. 5.20 is divided by $N(t)$.

b. The first term on the left-hand side of Eq. 5.20 contains the logarithmic derivative of the amplitude function, that is, the reciprocal period.

c. Nonlinear feedback effects make $D, A$, and $F^{T}$ functions of $\psi(\mathrm{x}, \mathrm{t}) \mathrm{N}(\mathrm{t})$.

The main assumption of the quasistatic method is now made. The time dependence of the shape function is not as important as that of the amplitude function. ${ }^{13}$ From a numerical standpoint, this means that only a few shape-function recalculations are needed, while the amplitude function is recalculated a great many times. Furthermore, the term $\partial \psi / \partial t$ in Eq. 5.20 can either be neglected entirely or replaced by the following backward difference approximation:

$$
\frac{\partial}{\partial t} \psi\left(x, t_{i}\right)=\frac{\psi\left(x, t_{i}\right)-\psi\left(x, t_{0}\right)}{t_{i}-t_{0}}
$$

Equation 5.22 assigns a linear variation to $\psi(x, t)$ over the first time interval. Neglecting $\partial \psi / \partial t$ entirely (the "original quasistatic approximation"15) assumes that the shape function $\psi\left(x, t_{0}\right)$ is used throughout the first interval. The following equation, called the "improved quasistatic approximation;" 15 results when Eq. 5.22 is substituted into Eq. 5.20:

$$
\begin{aligned}
\nabla \cdot D \nabla \psi\left(x, t_{i}\right)= & {\left[A-(1-\beta) \chi_{p} F^{T}+\left.\frac{1}{v N\left(t_{i}\right)} \frac{d N}{d t}\right|_{t=t_{i}}-\frac{1}{t_{i}-t_{0}}\right] \psi\left(x, t_{i}\right) } \\
& -\frac{\psi\left(x, t_{0}\right)}{v\left(t_{i}-t_{0}\right)}-\sum_{j=1}^{J} \frac{\lambda_{j} \chi_{j} \eta_{j}\left(x, t_{i}\right)}{N\left(t_{i}\right)} .
\end{aligned}
$$

The "adiabatic approximation" ${ }^{15}$ also neglects $\partial \psi / \partial \mathrm{t}$ in Eq. 5.20. Furthermore, it assumes that the shape of the delayed-neutron source is the same as that of the prompt-neutronsource. The new shape function is then found by solving the "lambda mode" eigenvalue problem, Eq. 3.39, for the fundamental lambda mode. The adiabatic model, then, removes all coupling between 
the amplitude calculation and the shape calculation, except for nonlinear feedback effects. Ott and Menele ${ }^{15}$ have shown that the adiabatic model is quite inferior to both the original quasistatic and the improved quasistatic methods. Nevertheless, it is far better than the point-reactor model; in which the shape function is never recalculated.

The residual of Eq. 5.9 can be significant in each of the quasistatic methods. In fact, even when Eq. 5.19 is used.to determine the initial condition of $\mathrm{N}_{2}$, the error introduced is so. large that the solution becomes meaningless. Therefore, an iterative scheme must be used to make $\mathrm{R}_{2}(\mathrm{x}) \equiv 0$ 。 The improved quasistatic method.implicitly contains a natural iterative scheme, which proceeds as follows:

For the initial guess, the shape $\psi\left(x, t_{i}\right)$ is estimated from Eq. 5.23. The second.iteration now proceeds over the first time interval with

$$
\psi_{1}(x, t)=\frac{t_{i}-t}{t_{i}-t_{0}} \psi_{1}\left(x, t_{0}\right)+\frac{t-t_{0}}{t_{i}-t_{0}} \psi_{1}\left(x, t_{i}\right)
$$

The shape function given by $E q .5 .24$ is used to calculate $\rho(t), \bar{\beta}_{j}(t)$, and $\Lambda^{\prime}(t)$ in the point-reactor model. When the amplitude function at $t=t_{i}$ has been recalculated, Eq. 5.23 is again used to get the shape function $\psi_{1}\left(x, t_{j}\right)$. Iterations are continued as needed; very few are actually. required. When a converged solution to $\phi\left(x, t_{i}\right)$ is obtained, the residual given.by Eq. 5.9. vanishes; that is,

$$
\psi_{2}\left(x, t_{i}\right)=\psi_{1}\left(x, t_{i}\right)
$$

and

$$
N_{2}\left(t_{i}\right)=N_{1}\left(t_{i}\right) \text {. }
$$

The solution proceeds over the second time.interval until another shape recalculation is needed.

Quasistatic methods have traditionally been formulated using the steady-state adjoint functions as the weighting functions. There is no intrinsic reason why this choice should be made, especially in the analysis of highly nonlinear transients. Becker ${ }^{44}$ has suggested the use of the timedependent adjoint function. This choice, however, requires a simultaneous solution of the adjoint problem. A more practical alternative is to use the time-dependent shape function itself as the weighting function. This application of the Galerkin method requires the satisfaction of the constraint condition, Eq. 3.24. 


\section{Shape-function. Improvement Using a. Nodal Method}

A possible way to increase the time-interval size between shapefunction recalculations is to apply the nodal method to the shape-function equation, ${ }^{64}$ that is, to Eq. 5.20. Since the precursor behavior must also be considered, Eq. 3.2 is rewritten as

$$
\frac{\partial \eta_{j}}{\partial t}(x, t)=\beta_{j} F^{T}(x, t) \psi(x, t) N(t)-\lambda_{j} \eta_{j}(x, t)
$$

For the firstiteration in the procedure described above, the reactor can be divided into nodes for purposes of carrying out the shape calculation. The shape function can now be expanded.in the approximate solution

$$
\bar{\psi}(\mathrm{x}, \mathrm{t})=\sum_{\mathrm{m}=1}^{\mathrm{M}} \theta_{\mathrm{m}}(\mathrm{x}) \mathrm{P}_{\mathrm{m}}(\mathrm{t}) \equiv \theta(\mathrm{x}) \mathrm{P}(\mathrm{t})
$$

where each $\theta_{\mathrm{m}}$ is the portion of the shape $\psi\left(x, t_{0}\right)$ appearing. in the mith node. Each $\mathrm{P}_{\mathrm{m}}(\mathrm{t})$ is then the "amplitude of the shape function" in the mth region. The matrices $\theta(x)$ and $P(t)$ are

$$
\theta(x)=\left[\theta_{1}(x) \ldots \theta_{M}(x)\right]
$$

and

$$
P(t) .=\left[\begin{array}{c}
P_{1}(t) \\
\cdot \\
\cdot \\
P_{M}(t)
\end{array}\right] \text {. }
$$

Equation 5.28 can now be substituted into Eqs. 5.20. and 5.27 to obtain the residuals

$$
\begin{aligned}
R_{F}(x, t)= & \frac{1}{v} \theta(x) \frac{d P}{d t} \\
& -\left[\nabla: D(x, t) \nabla-A(x, t)+(1-\beta) \chi_{p} F^{T}(x, t)-\frac{\alpha(t)}{v}\right] \theta(x) P(t) \\
& -\frac{1}{N(t)} \cdot \sum_{j=1}^{J} \lambda_{j} \chi_{j} \eta_{j}(x, t),
\end{aligned}
$$


and

$R_{P_{j}}(x, t)=\frac{\partial \eta_{j}}{\partial t}(x, t)-\beta_{j} F^{T}(x, t) \theta(x) P(t) N(t)+\lambda_{j} \eta_{j}(x, t)$,

where

$$
\alpha(t)=\frac{1}{N(t)} \frac{d N(t)}{d t}
$$

Introduce the weighting functions $\Omega(\mathrm{x})$,

$$
\Omega(\mathbf{x})=\left[\Omega_{1}(\mathbf{x}) \ldots \Omega_{\mathrm{M}}(\mathbf{x})\right]
$$

which will be used to weight Eqs. 5.31 and 5.32 in the method of weighted residuals. In a development closely resembling that of Chapter.IV, the following interface residuals and weighting functions are obtained:

$$
\begin{aligned}
& \mathrm{R}_{\mathrm{S}_{\mathrm{m}}}\left(\mathrm{x}_{\mathrm{m}}, \mathrm{t}\right)=\alpha_{1}\left[\theta_{\mathrm{m}+1}\left(\mathrm{x}_{\mathrm{m}}\right) \mathrm{P}_{\mathrm{m}+1}(\mathrm{t})-\theta_{\mathrm{m}}\left(\mathrm{x}_{\mathrm{m}}\right) \mathrm{P}_{\mathrm{m}}(\mathrm{t})\right] \\
& +\alpha_{2}\left[\left.D_{m+1}\left(x_{m}, t\right) \frac{d \theta_{m+1}^{\prime}}{d x}\right|_{x=x_{m}} P_{m+1}(t)\right. \\
& \left.-\left.D_{m}\left(x_{m}, t\right) \frac{d \theta_{m}}{d x}\right|_{x=x_{m}} P_{m}(t)\right] \\
& R_{D_{m}}\left(x_{m}, t\right)=\alpha_{1}\left[\theta_{m+1}\left(x_{m}\right) P_{m+1}(t)-\theta_{m}\left(x_{m}\right) P_{m}(t)\right] \\
& -\alpha_{2}\left[\left.D_{m+1}\left(x_{m}, t\right) \frac{d \theta m+1}{d x}\right|_{x=x_{m}}: P_{m+1}(t)\right. \\
& \left.-\left.D_{m}\left(x_{m}, t\right) \frac{d \theta_{m}}{d x}\right|_{x=x_{m}} P_{m}(t)\right] \text {, } \\
& \Omega_{\mathrm{S}_{\mathrm{m}}}\left(\mathrm{x}_{\mathrm{m}}\right)=\Omega_{\mathrm{m}}\left(\mathrm{x}_{\mathrm{m}}\right)
\end{aligned}
$$

and

$$
\Omega_{\mathrm{D}_{\mathrm{m}}}\left(\mathrm{x}_{\mathrm{m}}\right)=\Omega_{\mathrm{m}+1}\left(\mathrm{x}_{\mathrm{m}}\right), \quad \mathrm{m}=1, \ldots, \mathrm{M}-1
$$


To use the method of weighted residuals, note that

$$
\int_{a}^{b} \Omega^{T}(x) R_{F}(x, t) d x=\left[\begin{array}{c}
\int_{a}^{x_{1}} \Omega_{1}(\dot{x}) R_{F_{1}}(x, t) d x \\
\cdot \\
\int_{x_{M-1}}^{b} \Omega_{M}(x) R_{F_{M}}(x, t) d x
\end{array}\right]
$$

and

$$
\int_{a}^{b} \Omega^{T}(x) R_{P_{j}}(x, t) d x=\left[\begin{array}{cc}
\int_{a}^{x_{1}} \Omega_{1}(x) R_{P_{j 1}}(x, t) d x \\
\cdot \\
\int_{x_{M-1}}^{b}{ }^{b} M^{(x) R_{P}}{ }_{j M}^{(x, t) d x}
\end{array}\right]
$$

so that, analogous to Eqs. 4.25 and 4.26 ,

$$
\begin{aligned}
0= & \int_{x_{m-1}}^{x_{m}} \Omega_{m}(x) R_{F_{m}}(x, t) d x-\Omega_{m}\left(x_{m}\right) R_{S_{m}}\left(x_{m}, t\right) \\
& -\Omega_{m}\left(x_{m-1}\right) R_{D_{m-1}}\left(x_{m-1}, t\right),
\end{aligned}
$$

and

$\int_{x_{m-1}}^{x_{m}} \Omega_{m}(x) R_{P_{j m}}(x, t) d x=0, \quad m=1, \ldots, M, \quad j=1, \ldots, J$,

where

$$
\mathrm{x}_{0}=\mathrm{a} \text { and } \mathrm{x}_{\mathrm{M}}=\mathrm{b}
$$

The weighted-residual criteria, Eqs. 5.41 and 5.42, yield a set of first-order, ordinary differential equations, similar in form to the multimode kinetics equations. This system can be written in the compact form, 


$$
L \frac{d P}{d t}=K(t) P(t)+\frac{1}{N(t)} \sum_{j=1}^{J} \lambda_{j} Q_{j}(t)
$$

and

$$
\frac{d Q_{j}}{d t}=B_{j}(t) N(t) P(t)-\lambda_{j} Q_{j}(t),
$$

where the matrix elements of the I $x$ I diagonal matrices $L$ and $B_{j}(t)$ are given by

$$
L_{r r}=\int_{x_{r-1}}^{x_{r}} \Omega_{r}(x) \frac{1}{v} \theta_{r}(x) d x, \quad r=1, \ldots, M,
$$

and

$$
\begin{aligned}
& B_{j r r}(t)=\beta_{j} \int_{x_{r-1}}^{x_{r}} \Omega_{r}(x) F^{T}(x, t) \chi_{j} \theta_{r}(x) d x, \quad r=1, \ldots, M ; \\
& j=1, \ldots, J .
\end{aligned}
$$

The I x I matrix $\mathrm{K}(\mathrm{t})$, however, is not diagonal, but tridiagonal. The main diagonal elements are given by

$$
\begin{aligned}
& K_{r r}(t)=\int_{x_{r-1}}^{x_{r}} \Omega_{r}(x)\left[\nabla \cdot D_{r}(x, t) \nabla-A_{r}(x, t)+(1-\beta) \dot{\chi}_{p} F_{r}^{T}(x, t)-\frac{\alpha(t)}{v}\right] \theta_{r}(x) d x \\
& -\Omega_{r}\left(x_{r-1}\right)\left[\alpha_{1} \theta_{r}\left(x_{r-1}\right)+\left.\left(1-\alpha_{2}\right) D_{r}\left(x_{r-1}, t\right) \frac{d \theta_{r}}{d x}\right|_{x=x_{r-1}}\right] \\
& -\Omega_{r}\left(x_{r}\right)\left[\alpha_{1} \theta_{r}\left(x_{r}\right)-\left.\left(1-\alpha_{2}\right) D_{r}\left(x_{r}, t\right) \frac{d \theta_{r}}{d x}\right|_{x=x_{r}}\right], \quad r=1, \ldots, M
\end{aligned}
$$

The backward-coupling terms on the supradiagonal are 


$$
\begin{aligned}
& K_{r, r+1}(t)=\Omega_{r}\left(x_{r}\right)\left[\alpha_{1} \theta_{r+1}\left(x_{r}\right)+\left.\alpha_{2} D_{r+1}\left(x_{r}, t\right) \frac{d \theta_{r+1}}{d x}\right|_{x=x_{r}}\right] \\
& r=1, \ldots, M-1
\end{aligned}
$$

whereas the forward-coupling terms on the subdiagonal are

$$
\mathrm{K}_{\mathrm{r}+1, \mathrm{r}}(\mathrm{t})=\Omega_{\mathrm{r}+1}\left(\mathrm{x}_{\mathrm{r}}\right)\left[\alpha_{1} \theta_{\mathrm{r}}\left(\mathrm{x}_{\mathrm{r}}\right)-\left.\alpha_{2} \mathrm{D}_{\mathrm{r}}\left(\mathrm{x}_{\mathrm{r}}, \mathrm{t}\right) \frac{\mathrm{d} \theta_{\mathrm{r}}}{\mathrm{dx}}\right|_{\mathrm{x}=\mathrm{x}_{\mathrm{r}}}\right], \quad \mathrm{r}=1, \ldots, \mathrm{M}-.1
$$

Equations 5.43 and 5.44 are solved to determine the shape function $\psi(x, t)$; the point-reactor model is used for $N(t)$. Notice, however, that the systems are coupled, since $N(t)$ appears in Eq.s. 5.43 and 5.44. Fortunately, since $\psi(x, t)$ is slowly varying in time relative to $N(t)$, Eqs. 5.43 and 5.44 do not have to be solved whenever $N(t)$ is calculated. Another fortunate circumstance is that, in addition to the subintervals for performing the $\mathrm{N}(\mathrm{t})$ and the $\psi(\mathrm{x}, \mathrm{t})$ calculations, the quasistatic method provides an intermediate subinterval, which is used to calculate new functional forms for $\rho, \bar{\beta}_{j}$, and $\Lambda$. (This refinement is not discussed here, except as pertinent to the $P(t)$ calculation.) Whenever it is time to change the functional form of $\rho(t)$, new values of the elements of $P(t)$ and the $Q_{j}(t)$ can be found, particularly since new functional forms for $B_{j}(t)$ and $K(t)$ are also found at these times. An approximate form for $N(t)$ is also to be chosen to aid in the solution.

The nodal approach provides a much better "first guess" for the shape function at $t=t_{i}$ then for the time-independent first choice of $\psi$ described in Section C.l of this chapter. In addition, this approach can reduce the number of shape calculations needed to analyze a transient. Since the shape calculations are the most difficulty by far, substantial savings in computing time possibly can be made. The nodal approach is not needed for the second iteration, since a linear shape function can now be used. However, when the calculation passes to the next time interval, with the new shape, the nodal method can again be used for the first iteration. 


\section{CHAPTER VI}

\section{INTEGRATION OF THE MULTIMODE KINETICS EQUATIONS}

Chapters III, IV, and V were'concerned with various approximations to effect the spatial integration of the multigroup diffusion equations. Each of these approximations uses the method of weighted residuals to derive the multimode kinetics equations, which must be integrated to complete the solution.

The integral-equation technique of integrating the multimode kinetics equations is discussed in this chapter. Particular emphasis is placed on the method of undetermined parameters, since it is a weighted-residual technique. Piecewise polynomial functions ${ }^{31}$ are chosen as trial functions for $N(t)$, and three weighted-residual criteria are discussed. They are collocation weighting, subdomain weighting, and Galerkin weighting.

A. Formulations of Integral Equations

Conventional numerical-integration methods, such as finitedifference or Runge-Kutta methods, have proved to be poor choices for integrating the point-reactor model. Because the time derivative is multiplied by $1 / v$, very small time steps must be taken to ensure a stable solution. This is especially true for fast reactors, where $v \approx 10^{7} \mathrm{~cm} / \mathrm{sec}$ or more. Consequently, approximate methods based on integral-equation formulations have been developed, ${ }^{7-11,65}$ which successfully overcome the finitedifference time-step limitation. Most of these methods formally integrate the point-reactor model and approximate the slowly varying portions of the integrands with trial solutions. form,$^{3}$

It is possible to write the point-reactor model in the quasilinear

$$
\Lambda \frac{d N}{d t}+\alpha N(t)=[\rho(t)-\bar{\beta}(t)+\alpha] N(t)+\sum_{j=1}^{J} \lambda_{j} C_{j}(t)
$$

and

$$
\frac{d C_{j}}{d t}=\bar{\beta}_{j}(t) N(t)-\lambda_{j} C_{j}(t)
$$

where $\alpha$ is a parameter. Equations 6.1 and 6.2 are now formally integrated to obtain

$$
N(t)=N\left(t_{0}\right) \exp \left[-\alpha\left(t-t_{0}\right) / \Lambda+\int_{t_{0}}^{t}\left[\frac{\rho\left(t^{\prime}\right)-\bar{\beta}\left(t^{\prime}\right)+\alpha}{\Lambda} N\left(t^{\prime}\right)+\sum_{j=1}^{J} \lambda_{j} C_{j}\left(t^{\prime}\right)\right] \exp \left[-\alpha\left(t-t^{\prime}\right) / \Lambda\right] d t^{\prime}\right.
$$


and

$$
C_{j}(t)=C_{j}\left(t_{0}\right) \exp \left[-\lambda_{j}\left(t-t_{0}\right)\right]+\bar{\beta}_{j} \int_{t_{0}}^{t} N\left(t^{\prime}\right) \cdot \exp \left[-\lambda_{j}\left(t-t^{\prime}\right] d t^{\prime} .\right.
$$

The time-step limitation is overcome by approximating the slowly varying part in each integral. One such approximation ${ }^{65}$. is the Taylor series

$$
N\left(t_{0}+\tau\right)=N\left(t_{0}\right)+\tau N^{\prime}\left(t_{0}\right)+\frac{\tau^{2}}{2 !} N^{\prime \prime}\left(t_{0}\right)+\ldots
$$

where $\tau=t^{\prime}-t_{0}$. The integrals in Eqs. 6.3 and 6.4 are then evaluated term by term. The parameter $\alpha$ is arbitrary. A good choice has been found to be $\mathrm{b}^{65}$

$$
\alpha=\beta-\rho\left(t_{0}\right) .
$$

Another possible choice is 9

$$
\alpha=1 .
$$

Hansen's largest eigenvalue method ${ }^{7,8}$ also $c$ an be derived from the quasilinear form. In Hansen's method, the trial solution becomes

$$
\alpha=\beta-\rho(t),
$$

and

$$
N\left(t_{0}+\tau\right)=N\left(t_{0}\right) e^{\omega \tau},
$$

where $\omega$ is the largest root of the inhour equation for an average reactivity appropriate to each time step. In addition, Hansen also forms the following trial solutions for the precursors:

$$
C_{j}\left(t_{0}+\tau\right)=C_{j}\left(t_{0}\right) e^{\omega \tau} \text {. }
$$

The success of the method of undetermined parameters,$^{10,11}$ however, does not depend upon the value of $\alpha$, because it is not really a quasilinear method. Instead of writing Eq. 6.1 and formally carrying out the integration, we immediately assume a trial solution for $N(t)$ and substitute it into the point-reactor model. The resulting residual is then multiplied by weighting functions, and the method of weighted residuals is applied. This enables the undetermined parameters in the trial solution to be evaluated, thus completing the solution. Even if a remains in the formulation, it can be cancelled at any stage of the calculation. 
Instead of illustrating the method of undetermined parameters with the point-reactor model, we will use it to integrate the multimode kinetics equations. This is done in Section B below.

B. The Method of Undetermined Parameters reference:

The multimode kinetics equations are rewritten here for easy

$$
\Lambda \frac{d N}{d t}=[\rho(t)-\bar{\beta}(t)] N(t)+\sum_{j=1}^{J} \lambda_{j} C_{j}(t)
$$

and

$$
\frac{d C_{j}}{d t}=\bar{\beta}_{j}(t) N(t)-\lambda_{j} C_{j}(t)
$$

where

$$
\bar{\beta}(t)=\sum_{j=1}^{J} \bar{\beta}_{j}(t)
$$

In addition, $N(t)$ is an I-dimensional column vector of amplitude functions, the $C_{j}(t)$ are $I$-dimensional vectors of precursor amplitudes, and $\Lambda, \rho(t)$, $\bar{\beta}_{j}(t)$ are I $x$ I matrices, where $I$ is the number of modes used in the spatial integration.

The method of undetermined parameters is now used for the temporal integration. If Eq. 6.13 is substituted into Eq. 6.12, then

$$
\Lambda \frac{\mathrm{dN}}{\mathrm{dt}}=\rho(\mathrm{t}) \mathrm{N}(\mathrm{t})-\sum_{j=1}^{\mathrm{J}} \frac{\mathrm{dC}_{j}}{\mathrm{dt}}
$$

If the values of $\mathrm{dC}_{\mathrm{j}} / \mathrm{dt}$ were known, Eq. 6.14 could be integrated directly. Equations 6.13 can be formally integrated over the time interval $t_{i} \leq t \leq t_{i+i}$ as follows:

$C_{j}(t)=C_{j}\left(t_{i}\right) \exp \left[-\lambda_{j}\left(t-t_{i}\right)\right]+\int_{t_{i}}^{t} \bar{\beta}_{j}\left(t^{\prime}\right) N\left(t^{\prime}\right) \exp \left[-\lambda_{j}\left(t-t^{\prime}\right)\right] d t^{\prime}$

In addition, the following expressions for $\mathrm{dC}_{\mathrm{j}} / \mathrm{dt}$ can be obtained by differ - . entiating Eq. 6.15: 


$$
\begin{aligned}
\frac{d C_{j}}{d t}= & -\lambda_{j} C_{j}\left(t_{i}\right) \exp \left[-\lambda_{j}\left(t-t_{i}\right)\right]+\bar{\beta}_{j}(t) N(t) \\
& -\lambda_{j} \int_{t_{i}}^{t} \bar{\beta}\left(t^{\prime}\right) N\left(t^{\prime}\right) \exp \left[-\lambda_{j}\left(t-t^{\prime}\right)\right] d t^{\prime} .
\end{aligned}
$$

The system represented by Eqs. 6.14 and 6.16 can be solved in the interval $t_{i} \leq t \leq t_{i+1}$ by first expanding the amplitude functions in the trial functions $T_{k}(t)$ as follows:

$$
\bar{N}(t)=\sum_{k=0}^{K} A_{k} T_{k}(t)
$$

Each $T_{k}(t)$ is a scalar function of time, so that each $A_{k}$ is an I-dimensional column vector. The vector $A_{0}$ is the value of the amplitude function vector $\bar{N}\left(t_{i}\right)$, so that $T_{0}(t)$ is unity. Each element of each remaining $A_{k}$ must be determined to complete the solution.

If Eq. 6.16 is substituted into Eq. 6.14, the following equation results:

$$
\begin{aligned}
\Lambda \frac{d N}{d t}= & {[\rho(t)-\bar{\beta}(t)] N(t)=\sum_{j=1}^{J} \lambda_{j}\left[C_{j}\left(t_{i}\right) \exp \left[-\lambda_{j}\left(t-t_{i}\right)\right]\right.} \\
& \left.+\int_{t_{i}}^{t} \bar{\beta}_{j}\left(t^{\prime}\right) N\left(t^{\prime}\right) \exp \left[-\lambda_{j}\left(t-t^{\prime}\right)\right] d t^{\prime}\right] .
\end{aligned}
$$

The approximate solution, Eq. 6.17, can now be substituted into Eq. 6.18 to obtain the residual

$$
\begin{aligned}
R_{N}(t)= & \Lambda \frac{d \bar{N}}{d t}-[\rho(t)-\bar{\beta}(t)] \bar{N}(t)-\sum_{j=1}^{J} \lambda_{j}\left[C_{j}\left(t_{i}\right) \exp \left[-\lambda_{j}\left(t-t_{i}\right)\right]\right. \\
& \left.+\int_{t_{i}}^{t} \bar{\beta}_{j}\left(t^{\prime}\right) \bar{N}\left(t^{\prime}\right) \exp \left[-\lambda_{j}\left(t-t^{\prime}\right)\right] d t^{\prime}\right]
\end{aligned}
$$

The weighting functions $V_{k}(t), k=1, \ldots, K$, can be used in the fol lowing weighted-residual method to determine the unknown constants in the vectors $A_{k}$ : 


$$
\int_{t_{i}}^{t_{i+l}} v_{r}(t) R_{N}(t) d t=0, \quad r=1, \ldots, K
$$

Equation 6.20 is an example of the method of undetermined parameters. Once the parameters in the $A_{k}$ are determined, the calculation can be repeated over the next time step, with $\bar{N}\left(t_{i+1}\right)$ now serving as the vector $A_{0}$.

C. Piecewise Polynomials as Trial Solutions

The approximate solution to the multimode kinetics equations is a piecewise polynomial if, for each time step considered, the trial solution is expressed as a Kth-degree polynomial. ${ }^{31}$. The coefficients of the polynomials are constant over a time step, but differ from one time step to the next, hence the name piecewise polynomials.

The best known examples of piecewise polynomials are spline functions ${ }^{31}$ of degree $M$, which are defined as Mth-degree polynomials over each interval, such that the function and its first $M$ - 1 derivatives are continuous at each point where the polynomial coefficients change. These points are known as joints. The most commonly used spline functions are cubic splines, that is, $M=3$.

Spline functions are not used in the ensuing development. Instead, piecewise-polynomial functions are used for which the only continuity requirements demanded are for the amplitude functions, not for any of their derivatives. Such a choice has been successful in the past for point kinetics. ${ }^{10,11}$ It is now extended to multimode kinetics. In the time interval $t_{i} \leq t \leq t_{i+1}$, let the trial solution, Eq. 6.17 , be given by the polynomial

$$
\bar{N}(t)=\sum_{k=0}^{K} A_{k}\left(t-t_{i}\right)^{k}
$$

Then,

$$
\frac{d \bar{N}}{d t}=\sum_{k=1}^{K} k A_{k}\left(t-t_{i}\right)^{k-1}
$$

If the system under analysis is nonlinear, the matrices $\rho(t)$ and $\bar{\beta}_{j}(t)$ can be approximated over the time interval by

$$
\rho(t)=\rho\left(t_{i}\right)+\left(t-t_{i}\right)\left(\rho_{1}+2 \rho_{2} t_{i}\right)+\rho_{2}\left(t-t_{i}\right)^{2},
$$

and

$$
\bar{\beta}_{j}(t)=\bar{\beta}_{j}\left(t_{i}\right)+\left(t-t_{i}\right)\left(\bar{\beta}_{j i}+2 \bar{\beta}_{j 2} t_{i}\right)+\bar{\beta}_{j 2}\left(t-t_{i}\right)^{2}
$$


The I x I matrices $\rho_{1}, \rho_{2}, \bar{\beta}_{j 1}$, and $\bar{\beta}_{j 2}$ are evaluated by fitting results generated from the feedback model to the forms given by Eqs. 6.23 and 6.24 The feedback occurs through the matrix operators $D, A$, and $F^{T}$, which, for nonlinear systems, are functions of the neutron flux. Therefore, the matrix elements of $\rho(t)$ and $\bar{\beta}_{j}(t)$, as defined by Eqs. 3.29 and 3.30 , become functionals of the neutron flux. Eqs. 6.21-6.24 can now be substituted into the weighted-residual criterion, Eq. 6.20. The result is

$$
\begin{aligned}
0= & \int_{t_{i}}^{t_{i+1}} \sum_{k=1}^{K} V_{r}(t)\left\{\left[k \Lambda\left(t-t_{i}\right)^{k-1}-\rho\left(t_{i}\right)\left(t-t_{i}\right)^{k}-\left(\rho_{1}+2 \rho_{2} t_{i}\right)\left(t-t_{i}\right)^{k+1}-\rho_{2}\left(t-t_{i}\right)^{k+2}\right] A_{k} d t\right. \\
& \left.-\int_{t_{i}}^{t_{i+1}} V_{r}(t)\left[\rho\left(t_{i}\right)+\left(\rho_{1}+2 \rho_{2} t_{i}\right)\left(t-t_{i}\right)+\rho_{2}\left(t-t_{i}\right)^{2}\right] A_{0}-\sum_{j=1}^{J} \frac{d C_{j}}{d t}\right\} d t .
\end{aligned}
$$

Equation 6.25 yields IK simultaneous algebraic equations, from which the elements of each of the $A_{k}, k=1, \ldots, K$, can be found. The weighting functions, $V_{r}(t)$, must be specified before the solution can be obtained. Section D below considers three possibilities: collocation weighting, subdomain weighting, and Galerkin weighting.

\section{Choices of Weighting Functions}

Before the choices of weighting functions are considered individually, the I-functions, ${ }^{11}$ which appear in each type, are defined by

$$
I_{j, m}\left(\Delta t_{i}\right)=\int_{t_{i}}^{t_{i+1}} \exp \left[-\lambda_{j}\left(t_{i+1}-\tau\right)\right] \tau^{m} d \tau,
$$

where

$$
\Delta t_{i}=t_{i+1}-t_{i}
$$

Two useful properties of the I functions are

$$
\exp \left[-\lambda_{j} t\right]=1-\lambda_{j} I_{j, 0}(t)
$$

and

$$
I_{j, m}(t)=\frac{1}{\lambda_{j}}\left[t^{m}-m I_{j, m-1}(t)\right], \quad m \geq 1 .
$$

\section{Collocation Weighting}

The residual $R_{N}(t)$ is allowed to vanish at the $K$ points $t_{r}$, $r=1, \ldots, K$, all within the time interval. The weighting functions are the Dirac delta functions 


$$
\mathrm{V}_{\mathbf{r}}(\mathrm{t})=\delta\left(\mathrm{t}-\mathrm{t}_{\mathbf{r}}\right) ; \quad \mathrm{r}=1, \ldots, \mathrm{K}
$$

When Eqs. 6.21-6.24 are substituted into Eq. 6.16, and Eqs. 6.27 and 6.28 are used, the following is obtained (where $\Delta t=t-t_{i}$ ):

$$
\begin{aligned}
\frac{d C_{j}}{d t}= & -\lambda_{j} C_{j}\left(t_{i}\right) \exp \left[-\lambda_{j}(\Delta t)\right]+\left\{\bar{\beta}_{j}\left(t_{i}\right)\left[1-\lambda_{j} I_{j, 0}(\Delta t)\right]\right. \\
& +\left(\bar{\beta}_{j 1}+2 \bar{\beta}_{j 2} t_{i}\right)\left[\Delta t-\lambda_{j} I_{j, 1}(\Delta t)\right]+\bar{\beta}_{j 2}\left[(\Delta t)^{2}\right. \\
& \left.\left.-\lambda_{j} I_{j}, 2(\Delta t)\right]\right\} A_{0}+\sum_{k=1}^{K}\left\{\bar{\beta}_{j}\left(t_{j}\right)\left[(\Delta t)^{k}-\lambda_{j} I_{j, k}(\Delta t)\right]\right. \\
& +\left(\bar{\beta}_{j 1}+2 \bar{\beta}_{j 2} t_{i}\right)\left[(\Delta t)^{k+1}-\lambda_{j} I_{j, k+1}(\Delta t)\right] \\
& \left.+\bar{\beta}_{j 2}\left[(\Delta t)^{k+2}-\lambda_{j} I_{j, k+2}(\Delta t)\right]\right\} A_{k} .
\end{aligned}
$$

Equations 6.29 and $6.30 \mathrm{c}$ an now be used in the weighted-residual criterion, Eq. 6.25, to derive the algebraic equations from which the $A_{k}$ can be determined. These are

$$
\begin{aligned}
& 0=\sum_{k=1}^{K}\left(\Lambda k\left(\Delta t_{r}\right)^{k-1}-\left[\rho\left(t_{i}\right)\left(\Delta t_{r}\right)^{k}+\left(\dot{\rho}_{1}+2 \rho_{2} t_{i}\right)\left(\Delta t_{r}\right)^{k+1}\right.\right. \\
& \left.+\rho_{2}\left(\Delta t_{r}\right)^{k+2}\right]+\sum_{j=1}^{J}\left\{\bar{\beta}_{j}\left(t_{i}\right)\left[\Delta t_{r}-\lambda_{j} I_{j, k}\left(\Delta t_{r}\right)\right]\right. \\
& +\left(\bar{\beta}_{j 1}+2 \bar{\beta}_{j 2} t_{i}\right)\left[\left(\Delta t_{r}\right)^{k+1}-\lambda_{j} I_{j, k+1}\left(\Delta t_{r}\right)\right]+\bar{\beta}_{j 2}\left[\left(\Delta t_{r}\right)^{k+2}\right. \\
& \left.\left.\left.-\lambda_{j} I_{j, k+2}\left(\Delta t_{r}\right)\right]\right\}\right) A_{k}-\left(\rho\left(t_{i}\right)+\left(\rho_{1}+2 \rho_{2} t_{i}\right) \Delta t_{r}\right. \\
& +\rho_{2}\left(\Delta t_{r}\right)^{2}-\sum_{j=1}^{J}\left\{\bar{\beta}_{j}\left(t_{j}\right)\left[1-\lambda_{j} I_{j, o}\left(\Delta t_{r}\right)\right]\right. \\
& +\left(\bar{\beta}_{j_{1}}+2 \bar{\beta}_{j 2} t_{i}\right)\left[\Delta t_{r}-\lambda_{j} I_{j, 1}\left(\Delta t_{r}\right)\right]+\bar{\beta}_{j 2}\left[\left(\Delta t_{r}\right)^{2}\right. \\
& \left.\left.\left.-\lambda_{j} I_{j, 2}\left(\Delta t_{r}\right)\right]\right\}\right) A_{0}-\sum_{j=1}^{J} \lambda_{j} C_{j}\left(t_{i}\right)\left[1-\lambda_{j} I_{j, o}\left(\Delta t_{r}\right)\right]
\end{aligned}
$$


where

$$
\Delta t_{r}=\frac{\Delta t}{2^{r-1}}=t_{r}-t_{i}, \quad r=1, \ldots, K
$$

The choice of collocation points given by Eq. 6.32 lends itself very well to time-step halving procedures, which control the error accumulation to ensure a stable solution. One such procedure is described in Appendix $B$. When the vectors $A_{k}$ are determined using Eq. 6.31, the values $\lambda_{j} C_{j}\left(t_{i+1}\right)$ can be determined directly from Eq. 6.12 by substituting the values $\vec{N}\left(t_{i+1}\right), d \bar{N} / d t\left(t_{i+1}\right), \rho\left(t_{i+1}\right)$, and $\beta\left(t_{i+1}\right)$ just obtained. The calculation can now be repeated for the next time step.

\section{Subdomain Weighting}

The subdomain method was first used for point kinetics by Brittan ${ }^{10}$ and Kaganove. ${ }^{11}$ The weighting functions are the unit step functions

$$
V_{r}(t)=U(t)-U\left(t-t_{r}\right), \quad r=1, \ldots, K
$$

where each $t_{r}$ is given by Eq. 6.32. The subdomains are therefore the whole time interval, the half-interval, the quarter-interval, etc., until $\mathrm{K}$ intervals have been chosen. As with the collocation method, the time-step halving procedure described in Appendix B can easily be used for this choice of subdomains.

For the rth subdomain, the limits of integration in Eq. 6.25 become the points $t_{i}$ and $t_{r}$. For each of the $K$ resulting equations,

$$
\int_{t_{i}}^{t_{r}} \frac{d C_{j}}{d t} d t=C_{j}\left(t_{r}\right)-C_{j}\left(t_{i}\right) .
$$

Equations 6.13 and $6.30 \mathrm{c}$ an be combined to obtain

$$
\begin{aligned}
C_{j}\left(t_{r}\right)= & C_{j}\left(t_{i}\right) \exp \left[-\lambda_{j} \Delta t_{r}\right]+\left[\bar{\beta}_{j}\left(t_{i}\right) I_{j, 0}\left(\Delta t_{r}\right)+\left(\bar{\beta}_{j 1}+2 \bar{\beta}_{j 2} t_{i}\right) I_{j, 1}\left(\Delta t_{r}\right)+\bar{\beta}_{j 2} I_{j, 2}\left(\Delta t_{r}\right)\right] A_{0} \\
& +\sum_{k=1}^{K}\left[\bar{\beta}_{j}\left(t_{i}\right) i_{j, k}\left(\Delta t_{r}\right)+\left(\bar{\beta}_{j 1}+2 \bar{\beta}_{j 2} t_{i}\right) I_{j, k+1}\left(\Delta t_{r}\right)+\bar{\beta}_{j 2} I_{j, k+2}\left(\Delta t_{r}\right)\right] A_{k},
\end{aligned}
$$

which, in turn, can be substituted back into Eq. 6.34. When the result is substituted into Eq. 6.24, together with Eqs. 6.21-6.24 and 6.33, the following is obtained: 


$$
\begin{aligned}
0= & \sum_{k=1}^{K}\left\{\Lambda\left(\Delta t_{r}\right)^{k}-\frac{\rho\left(t_{i}\right)\left(\Delta t_{r}\right)^{k+1}}{k+1}+\frac{\left(\rho_{1}+2 \rho_{2} t_{i}\right)\left(\Delta t_{r}\right)^{k+2}}{k+2}+\frac{\rho_{2}\left(\Delta t_{r}\right)^{k+3}}{k+3}\right. \\
& \left.+\sum_{j=1}^{J}\left[\bar{\beta}_{j}\left(t_{i}\right) I_{j, k}\left(\Delta t_{r}\right)+\left(\bar{\beta}_{j_{1}}+2 \bar{\beta}_{j 2} t_{i}\right) I_{j, k+1}\left(\Delta t_{r}\right)+\bar{\beta}_{j 2} I_{j, k+2}\left(\Delta t_{r}\right)\right]\right\} A_{k} \\
& -\left\{\left[\rho\left(t_{i}\right) \Delta t_{r}+\frac{\left(\rho_{1}+2 \rho_{2} t_{i}\right)\left(\Delta t_{r}\right)^{2}}{2}+\frac{\rho_{2}\left(\Delta t_{r}\right)^{3}}{3}\right]\right. \\
& \left.-\sum_{j=1}^{J}\left[\bar{\beta}_{j}\left(t_{i}\right) I_{j, 0}\left(\Delta t_{r}\right)+\left(\bar{\beta}_{j_{1}}+2 \bar{\beta}_{j 2} t_{i}\right) I_{j, 1}\left(\Delta t_{r}\right)+\bar{\beta}_{j 2} I_{j, 2}\left(\Delta t_{r}\right)\right]\right\} A_{0} \\
& -\sum_{j=1}^{J} \lambda_{j} C_{j}\left(t_{i}\right) I_{j, 0}\left(\Delta t_{r}\right), \quad r=1, \ldots, K .
\end{aligned}
$$

Equation 6.36 represents IK simultaneous equations, which can be solved to determine the elements of each vector $A_{k}$. The values of $\mathrm{C}_{\mathrm{j}}\left(t_{\mathrm{i}+1}\right)$ can be found using Eq. 6.12, as was outlined for collocation weighting in Section D.l above.

\section{Galerkin Weighting}

For the Galerkin method, the weighting functions are the trial functions; that is,

$$
V_{r}(t)=\left(t-t_{i}\right)^{r}, \quad r=1, \ldots, K
$$

Consequently, each weighting function is continuous over the entire time interval. Furthermore, terms of the form

$$
J_{j, r}\left(\Delta t_{i}\right)=\int_{t_{i}}^{t_{i+1}}\left(t-t_{i}\right)^{r} \frac{d C_{j}}{d t} d t
$$

appear in the weighted-residual criterion, Eq. 6.25. These integrals can be evaluated with the aid of a recursion formula, which will be derived using partial integration. 
To begin the derivation, observe that

$$
J_{j, 0}\left(\Delta t_{i}\right)=C_{j}\left(t_{i+1}\right)-C_{j}\left(t_{i}\right)
$$

Equation 6.38 can be integrated by parts to obtain

$$
J_{j, r}\left(\Delta t_{i}\right)=\left(\Delta t_{i}\right)^{r} C_{j}\left(t_{i+1}\right)-r \int_{t_{i}}^{t_{i+1}}\left(t-t_{i}\right)^{r-1} C_{j}(t) d t
$$

If Eq. 6.13 is used to eliminate $C_{j}(t)$ in the integrand, and then Eq. 6.38 is used, Eq. 6.40 becomes

$J_{j, r}\left(\Delta t_{i}\right)=\left(\Delta t_{i}\right)^{r} C_{j}\left(t_{i+1}\right)-\frac{r}{\lambda_{j}} \int_{t_{i}}^{t_{i+1}}\left(t-t_{i}\right)^{r-1} \bar{\beta}_{j}(t) N(t) d t+\frac{r}{\lambda_{j}} J_{j, r-1}\left(\Delta t_{i}\right)$.

When Eqs. 6.21 and 6.24 are used, the following recursion formula can be obtained from Eq. 6.41 :

$$
\begin{aligned}
J_{j, r}\left(\Delta t_{i}\right)= & \left(\Delta t_{i}\right)^{r} C_{j}\left(t_{i+1}\right)-\frac{r}{\lambda_{j}} \sum_{k=0}^{K}\left[\frac{\bar{\beta}_{j}\left(t_{j}\right)\left(\Delta t_{i}\right)^{k+r}}{k+r}+\frac{\left(\bar{\beta}_{j 1}+2 \bar{\beta}_{j 2} t_{i}\right)\left(\Delta t_{i}\right)^{k+r+1}}{k+r+1}+\frac{\bar{\beta}_{j 2}\left(\Delta t_{i}\right)^{k+r+2}}{k+r+2}\right] A_{k} \\
& +\frac{r}{\lambda_{j}} J_{j, r-1}\left(\Delta t_{i}\right), \quad r=1, \ldots, K .
\end{aligned}
$$

The weighted-residual method is now applied by substituting Eqs. 6.21-6.24 and 6.37 into Eq. 6.25 to obtain

$$
\begin{aligned}
0= & \sum_{k=1}^{K}\left[\frac{\Lambda k\left(\Delta t_{i}\right)^{k+r}}{k+r}-\frac{\rho\left(t_{i}\right)\left(\Delta t_{i}\right)^{k+r+1}}{k+r+1}+\frac{\left(\rho_{1}+2 \rho_{2} t_{i}\right)\left(\Delta t_{i}\right)^{k+r+2}}{k+r+2}+\frac{\rho_{2}\left(\Delta t_{i}\right)^{k+r+3}}{k+r+3}\right] A_{k} \\
& -\left[\frac{\rho\left(t_{i}\right)\left(\Delta t_{i}\right)^{r+1}}{r+1}+\frac{\left(\rho_{1}+2 \rho_{2} t_{i}\right)\left(\Delta t_{i}\right)^{r+2}}{r+2}+\frac{\rho_{2}\left(\Delta t_{i}\right)^{r+3}}{r+3}\right] A_{0}+\sum_{j=1}^{J} J_{j, r}\left(\Delta t_{i}\right), \quad r=1, \ldots, K,
\end{aligned}
$$

where the $J_{j, r}\left(\Delta t_{j}\right)$ are determined from the recursion formula, Eq. 6.42. The recursion relationship must be used for each value of $r$ to obtain the equations necessary to solve for the $A_{k}$ explicitly. This is not difficult to do when using a digital computer. Indeed, recursion formulas are ideally suited for digital computation. The time-step halving procedure can still be used in conjunction with the Galerkin equations, that is, Eqs. 6.43 and 6.42, but it is not intrinsically connected with the choice of weighting functions, as with collocation and subdomain weighting. 


\section{CHAPTER, VII}

SPACE-TIME ITERATION USING THE METHOD OF UNDETERMINED FUNCTIONS

A. Description of the Method

In Chapters III, IV, and V, the method of undetermined functions was us ed to perform spatial integrations in derivations of the multimode kinetics equations. Then, in Chapter VI, the method of undetermined parameters was used to integrate the multimode kinetics equations, thus completing the solution to the space-time problem. In this chapter, an iterative method, ${ }^{32}$ which uses the method of undetermined functions, will be applied to perform both spatial and temporal integrations. The method, described in detail in Chapter II, yields solutions that are independent of the initial choices of trial functions. Accuracy of the solutions thus depends only upon the number of trial functions chosen.

The technique of space-time iteration begins by applying the method of weighted residuals to Eqs. 3.1 and 3.2 to obtain the multimode kinetics: equations (see Chapter III). These are then solved for the amplitude functions, presumably by using one of the methods discussed in Chapter VI. The amplitude functions then serve as trial functions in a semidirect method to determine new values for the time-dependent shape functions.

Since the amplitude functions are determined in Chapter III, only the method of recalculating the shape functions is outlined in this chapter. A one-energy group model is used for simplicity. The precursor equations can be formally integrated with respect to the time variable to obtain

$\eta_{j}(x, t)=\eta_{j}\left(x, t_{0}\right) \exp \left[-\lambda_{j}\left(t-t_{0}\right)\right]+\beta_{j} \int_{t_{0}}^{t} F^{T}\left(x, t^{\prime}\right) \phi\left(x, t^{\prime}\right) \exp \left[-\lambda_{j}\left(t-t^{\prime}\right)\right] d t^{\prime}$.

Equation 7.1 can then be substituted into Eq. 3.1, which becomes

$$
\begin{aligned}
\frac{1}{v} \frac{\partial \phi(x, t)}{\partial t}= & {\left[\nabla \cdot D(x, t) \nabla-A(x, t)+(1-\beta) \chi_{p} F^{T}(x, t)\right] \phi(x, t) } \\
& +\sum_{j=1}^{J}\left[\eta_{j}\left(x, t_{0}\right) \exp \left[-\lambda_{j}\left(t-t_{0}\right)\right]+\beta_{j} \int_{t_{0}}^{t} F^{T}\left(x, t^{\prime}\right) \phi\left(x, t^{\prime}\right) \exp \left[-\lambda_{j}\left(t-t^{\prime}\right)\right] d t^{\prime}\right] .
\end{aligned}
$$

To perform the temporal weighted-residual method, the following weighting functions are introduced:

$$
T(t)=\left[T_{1}(t) \ldots T_{I}(t)\right]
$$


The weighted-residual method can now be applied in the usual manner to Eq. 7.2 to obtain

$$
\begin{aligned}
\int_{t_{0}}^{t_{f}} T^{T}(t) \frac{1}{v} \psi(x) \frac{d N}{d t} d t= & \int_{t_{0}}^{t_{f}} T^{T}(t)\left\{\left[\nabla \cdot D \nabla-A+(1-\beta) \chi_{p} F^{T}\right] \psi(x) N(t)\right. \\
& \left.+\sum_{j=1}^{J} \beta_{j} \int_{t_{0}}^{t} F^{T}\left(x, t^{\prime}\right) \psi(x) N\left(t^{\prime}\right) \exp \left[-\lambda_{j}\left(t-t^{\prime}\right)\right] d t^{\prime}\right\} d t \\
& +\sum_{j=1}^{J} \int_{t_{0}}^{t_{f}} T^{T}(t) \eta_{j}\left(x, t_{0}\right) \exp \left[-\lambda_{j}\left(t-t_{0}\right)\right] d t .
\end{aligned}
$$

Equation 7.4 is used to solve for the shape functions $\psi_{i}, i=1, \ldots, I$, appearing in the matrix $\psi(x)$. For a one-energy group approximation, Eq. 7.4 can be rewritten as

$$
\begin{aligned}
0= & \int_{t_{0}}^{t_{f}} T^{T}(t)\left\{\frac{1}{v} \frac{d N^{T}}{d t}-\left[\nabla \cdot D(x, t) \nabla-A(x, t)+(1-\beta) x_{p} F^{T}(x, t)\right] N^{T}(t)\right. \\
& \left.+\sum_{j=1}^{J} \beta_{j} \int_{t_{0}}^{t} F^{T}\left(x, t^{\prime}\right) N^{T}\left(t^{\prime}\right) \exp \left[-\lambda_{j}\left(t-t^{\prime}\right)\right] d t^{\prime}\right\} d t \psi^{T}(x) \\
& +\sum_{j=1}^{J} T^{T}(t) \eta_{j}\left(x, t_{0}\right) \exp \left[-\lambda_{j}\left(t-t_{0}\right)\right] d t
\end{aligned}
$$

Furthermore, Eq. 7.5 can be written in the matrix shorthand form

$$
\frac{d}{d x}\left[E(x) \frac{d \psi^{T}}{d x}(x)\right]+M(x) \psi^{T}(x)+P(x)=0
$$

where the I $x$ I matrices $E(x)$ and $M(x)$ and the I-dimensional column vector $P(x)$ are defined by

$$
E(x)=\int_{t_{0}}^{t_{f}} T^{T}(t) D(x, t) N^{T}(t) d t
$$




$$
\begin{aligned}
M(x)= & \int_{t_{0}}^{t_{f}} T^{T}(t)\left\{\left[(1-\beta) \chi_{p} F^{T}(x, t)-A(x, t)-\frac{1}{v} \frac{d}{d t}\right] N^{T}(t)\right. \\
& \left.+\sum_{j=1}^{J} \beta j \int_{t_{0}}^{t} F^{T}\left(x, t^{\prime}\right) N^{T}\left(t^{\prime}\right) \exp \left[-\lambda_{j}\left(t-t^{\prime}\right)\right] d t^{\prime}\right\} d t,
\end{aligned}
$$

and

$$
P(x)=\sum_{j=1}^{J} \eta_{j}\left(x, t_{0}\right) \int_{t_{0}}^{t_{f}} T^{T}(t) \exp \left[-\lambda_{j}\left(t-t_{0}\right)\right] d t
$$

Equation 7.6 is similar to the matrix formulation of steady-state multigroup diffusion theory. One vital difference, however, is that in multigroup diffusion theory, $\mathrm{E}(\mathrm{x})$ is a diagonal matrix, whereas in $\mathrm{Eq}$. 7.6, it is a full matrix. Nevertheless, Eq. 7.6 can be solved as a system of inhomogeneous, second-order, ordinary differential equations to obtain the new values of $\psi_{i}(x)$. These new values can, in turn, serve as trial functions to recalculate the amplitude functions $N_{i}(t)$, using the method described in Chapter III. The iteration is carried out until a converged solution is obtained.

\section{B. A Simple Illustration}

Consider the one-dimensional bare reactor shown in Fig. 3. Suppose it is initially critical, but then a uniform step decrease in the absorption.

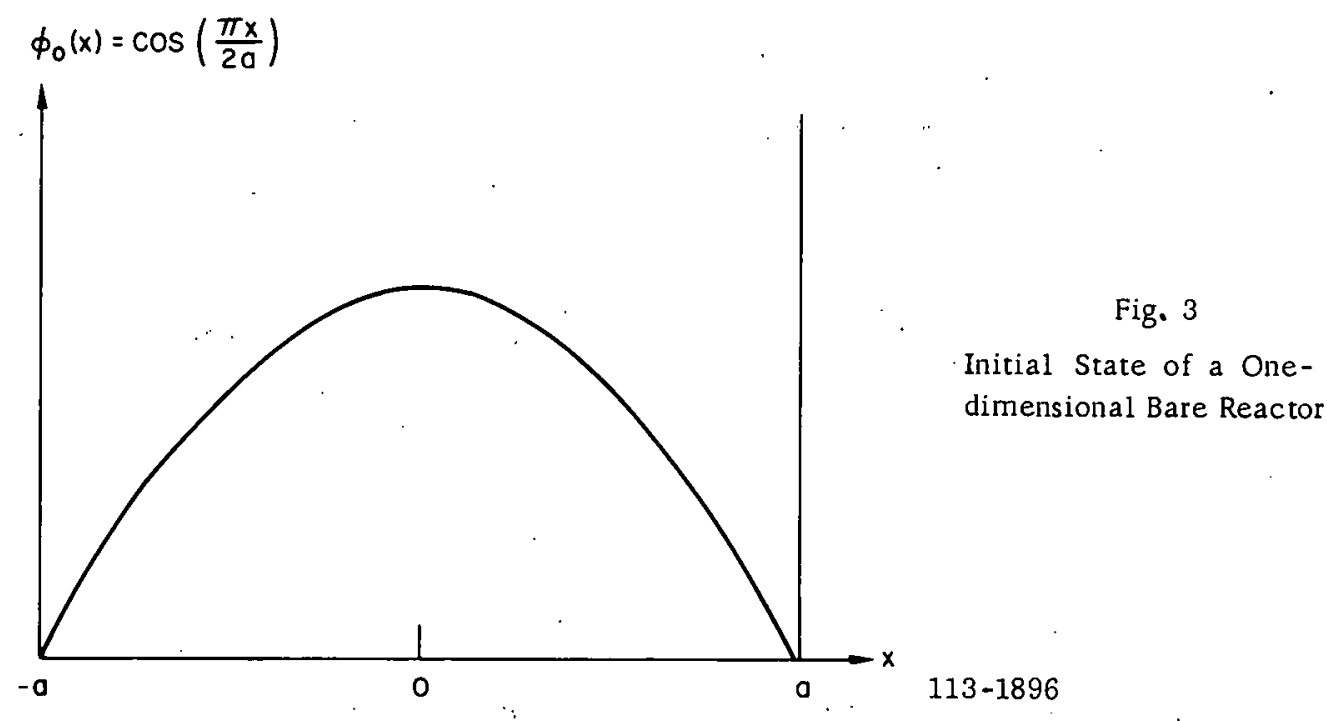


cross section takes place. Neglect the delayed neutrons for simplicity, so that Eqs. 3.1 and 3.12 become

$$
\frac{1}{v} \frac{\partial \phi(x, t)}{\partial t}=D \frac{\partial^{2} \phi(x, t)}{\partial x^{2}}+\left(F^{T}-A\right) \phi(x, t)
$$

and

$$
\phi(-\mathrm{a}, \mathrm{t})=\phi(\mathrm{a}, \mathrm{t})=0
$$

Since the reactor is initially critical, the initial condition for the flux is

$$
\phi(x, 0)=\phi_{0}(x)=\cos \frac{\pi x}{2 a}
$$

It is well known that, for a uniform perturbation, the flux shape will not change. Nevertheless, a one-mode approximation is chosen, in which the trial function is not the initial distribution $\phi_{0}(\mathbf{x})$. Instead, choose

$$
\bar{\phi}=\psi(\mathrm{x}) \mathrm{N}(\mathrm{t})
$$

where

$$
\psi(\mathbf{x})=1-\frac{x^{2}}{a^{2}}
$$

The trial function satisfies the boundary conditions, so that a boundary residual need not be formed. The object of this example is to show that the iterative scheme seeks and finds the exact solution, even though an incorrect shape function is used as the initial guess.

The amplitude function $\mathrm{N}(\mathrm{t})$ can be found by solving the multimode kinetics equations. The initial condition $N\left(t_{0}\right)$ is found by using $\mathrm{Eq} .3 .20$. Choose subdomain weighting, $W(x)=1$, so that Eq. 3.20 becomes

$$
\int_{-a}^{\dot{a}}\left[\cos \frac{\pi x}{2 a}-\left(1-\frac{x^{2}}{a^{2}}\right) N\left(t_{0}\right)\right] d x=0
$$

from which

$$
N\left(t_{0}\right)=\frac{3}{\pi}
$$

The generation time and the reactivity are found from Eqs. 3.28 and 3.29 , so that 


$$
\Lambda=\int_{-a}^{a} \frac{1}{v}\left(1-\frac{x^{2}}{a^{2}}\right) d x=\frac{4 a}{3 v}
$$

and

$$
\rho=\int_{-a}^{a}\left[-\frac{2 D}{a^{2}}+\left(F^{T}-A\right)\left(1-\frac{x^{2}}{a^{2}}\right)\right] d x=-\frac{4 D}{a}+\frac{4 a}{3}\left(F^{T}-A\right) .
$$

If Eq. 7.18 is divided by Eq. 7.17, the result is

$$
\frac{\rho}{\Lambda}=v\left(F^{T}-A-\frac{3 D}{a^{2}}\right)
$$

Equation 3.32 can now be easily solved for the amplitude function $N(t)$. The solution is

$N(t)=N\left(t_{0}\right) \exp \left[(\rho / \Lambda)\left(t-t_{0}\right)\right]=\frac{3}{\pi} \exp \left[v\left(F^{T}-A-3 D / a^{2}\right)\left(t-t_{0}\right)\right]$

The first guess for the flux is thus

$$
\phi(x, t)=\frac{3}{\pi}\left(1-\frac{x^{2}}{a^{2}}\right) \exp \left[v\left(F T-A-3 D / a^{2}\right)\left(t-t_{0}\right)\right]
$$

The improved solution for $\psi(\mathrm{x})$ will now be sought, using Eq. 7.20 as a trial function in Eq. 7.6.

Since there are no delayed neutrons, $P(x)=0$, and Eq. 7.6 is homogeneous. An eigenvalue problem consequently must be formed to solve Eq. 7.6. To evaluate $E(x)$ and $M(x)$ (both constant for this example), the weighting function $T(t)=1$ will be chosen. This choice corresponds to subdomain weighting. Therefore, $E(x)$ and $M(x)$ become

$$
E(x)=\frac{D N\left(t_{0}\right)}{\rho / \Lambda}\left\{\exp \left[(\rho / \Lambda)\left(t_{f}-t_{0}\right)\right]-1\right\}
$$

and

$$
M(x)=N\left(t_{0}\right)\left(\frac{F^{T}-A}{\rho / \Lambda}\left\{\exp \left[(\rho / \Lambda)\left(t_{f}-t_{0}\right)\right]-1\right\}-\frac{1}{v}\left\{\exp \left[(\rho / \Lambda)\left(t_{f}-t_{0}\right)\right]-1\right\}\right)
$$


so that Eq. 7.6 becomes

$$
\frac{\mathrm{d}^{2} \psi}{d \mathrm{x}^{2}}+\left[\frac{\mathrm{F}^{\mathrm{T}}-\mathrm{A}}{\mathrm{A}}-\frac{\rho / \Lambda}{\mathrm{vD}}\right] \psi(\mathrm{x})=0 .
$$

Equation 7.24 is a homogeneous equation, which recurs in each spatial iteration. To obtain a nontrivial solution, introduce a parameter $\lambda^{2}$ in the following manner:

$$
\frac{\mathrm{d}^{2} \psi}{\mathrm{d} \mathrm{x}^{2}}+\left[\frac{\mathrm{F}^{\mathrm{T}}-\mathrm{A}}{\mathrm{D}}-\frac{\rho / \Lambda}{\mathrm{vD}}-\lambda^{2}\right] \psi(\mathbf{x})=0 .
$$

The parameter $\lambda^{2}$ is evaluated for each iteration by using the boundary conditions. As the iterative scheme progresses, each succeeding value of $\lambda^{2}$ should be smaller than the preceding value. If $\lambda^{2}$ vanishes, then the converged solution has been obtained. Equation 7.25 , then, amounts to an "eigenvalue problem" for each iteration.

Equation $7.19 \mathrm{can}$ be substituted into Eq. 7.25 to obtain

$$
\frac{\mathrm{d}^{2} \psi}{\mathrm{dx}}+\left(\frac{3}{\mathrm{a}^{2}}-\lambda^{2}\right) \psi(\mathrm{x})=0
$$

By requiring the boundary condition, Eq. 7.11 , to be satisfied, one can show that the solution is

$$
\begin{aligned}
& \psi(x)=C \cos \frac{\pi x}{2 a}, \\
& \lambda^{2}=\frac{3-\pi^{2} / 4}{a^{2}},
\end{aligned}
$$

and

$$
\phi(x, t)=C \cos \frac{\pi x}{2 a} \exp \left[v\left(F^{T}-A-3 D / a^{2}\right) t\right]
$$

Finally, the constant $C$ is evaluated by applying the weighted-residual method to the initial residual:

$$
\int_{-a}^{a} \cos \frac{\pi x}{2 a}-C \cos \frac{\pi x}{2 a} d x=0
$$

from which $C=1$. 
Further improvement can now be made for $N(t)$ by using the trial function

$$
\psi(x)=\cos \frac{\pi x}{2 a}
$$

The solution proceeds along the same lines as outlined in connection with Eqs. 7.17-7.21. This time, however,

$$
\begin{aligned}
& \rho / \Lambda=v\left(F^{T}-A-\frac{\pi^{2} D}{4 a^{2}}\right), \\
& N(t)=\exp \left[v\left(F^{T}-A-\frac{\pi^{2} D}{4 a^{2}}\right)\left(t-t_{0}\right)\right],
\end{aligned}
$$

and

$$
\phi(x, t)=\cos \frac{\pi x}{2 a} \exp \left[v\left(F^{T}-A-\frac{\pi^{2} D}{4 a^{2}}\right)\left(t-t_{0}\right)\right] .
$$

Equation 7.34 is the well-known exact solution to the problem. If this fact were not known, however, another calculation of the shape function would again yield the solution given by Eq. 7.27. The parameter $\lambda^{2}$ would now vanish, indicating that the converged solution has been found.

C. Possible Applications of Space-Time Iteration

Space-time iteration is ideally suited for use with temporally discontinuous trial functions in the analysis of nonlinear transients. In the quasistatic method, for example, space-time iteration could be used to improve the shape function over the ith time step, instead of using Eq. 5.24. Since there is only one mode, however, the shape function would be characteristic of an instant in time somewhere within the ith time interval. This fact suggests that space-time iteration with only one shape function is inferior to using Eq. 5.24. However, if more than one time-independent mode were used, as in temporally discontinuous synthesis methods, then space-time iteration would be an extremely valuable tool because it eliminates the need to choose a "good" set of trial functions.

Another possible application could be to use space-time iteration in 'the full three-dimensional, time-dependent problem. Trial functions could initially be chosen in the three space dimensions in order to calculate the amplitude functions. Then, the amplitude functions, together with the trial functions in two of the space dimensions, could be used to improve the shape functions in the remaining space dimension. The procedure would then be 
continued to improve the shape functions in each remaining space dimension. Then, the second iteration would begin by calculating improved amplitude functions using the improved shape functions as trial functions, and would continue by recalculating the shape functions for each dimension in turn. The procedure would become, in effect, a series of one-dimensional calculations performed to solve a four-dimensional problem. When a nonlinear transient is analyzed, the temporally discontinuous trial function technique could also be incorporated.

Multichannel-synthesis methods could also benefit from space-time iteration. In the nodal method, for example, shape functions characteristic of an instant of time within the time interval would be used instead of the initial shapes. Each node would presumably be described by a shape function characteristic of a time different from that of the other shapes. 


\section{CHAPTER VIII \\ NUMERICAL RESULTS}

A. Introduction

In the previous chapters the approximate methods of solving the space-time problem were described as weighted-residual methods, differing only in the manner of choosing trial functions. Methods of choosing weighting functions were not compared, except for occasional implications that the variational method and the Galerkin method seemed to be superior to other choices. In this chapter, various methods of choosing weighting functions are compared numerically. The results are primarily intended to indicate the degree of sensitivity of the weighted-residual solutions to the choice of weighting functions.

A computer program, entitled WEIRD, was written to perform the calculations. It integrates the one-energy-group diffusion equation. Trial functions and weighting functions for the space dependence are inputs. The multimode kinetics equations are then formed and integrated using the method of undetermined parameters. Piecewise polynomials are used as the trial functions for the time integration. Second-degree polynomials and subdomain weighting are used to obtain the results that will be reported. The time-integration routine also has an automatic time-step selector, which is described in Appendix B.

Comparisons are made for two of the approximate methods. These are continuous synthesis and multichannel synthesis. Nodal methods are treated as special cases of multichannel synthesis. In the studies of continuous synthesis, the Galerkin method is compared with two choices of subdomain weighting and with a set of weighting functions that satisfy the boundary conditions but are otherwise chosen somewhat arbitrarily. No more than two trial functions are considered for any problem. Studies of the multichannel synthesis method indicate the degree of improvement in the approximation when the number of synthesis regions is increased. In addition, partial-current weighting and surface-cancellation weighting of the interface conditions are compared for the nodal method.

Only one reactor is analyzed in this study. It is a $240-\mathrm{cm}$ slab reactor, which, for convenience, is treated in a symmetric manner. That is, the only transients studied result from perturbations symmetric about the center of the reactor so that only half the reactor is considered. Hence, a zero-current boundary condition is imposed at the origin. The parameters for criticality are listed in Table $I$, and the initial flux shape is shown in Fig. 4 . 
TABLE I. Parameters for Criticality

\begin{tabular}{lccc}
\hline Parameter & $\begin{array}{c}\text { Region 1 } \\
(0-15 \mathrm{~cm})\end{array}$ & $\begin{array}{c}\text { Region 2 } \\
(15-60 \mathrm{~cm})\end{array}$ & $\begin{array}{c}\text { Region 3 } \\
(60-120 \mathrm{~cm})\end{array}$ \\
\hline $\mathrm{D}, \mathrm{cm}$ & 1.69531 & 1.69531 & 1.69531 \\
$\nu \Sigma_{\mathrm{f}}, \mathrm{cm}^{-1}$ & 0.0194962 & 0.0194962 & 0.0194962 \\
$\Sigma_{\mathrm{a}}, \mathrm{cm}^{-1}$ & 0.0194962 & 0.0194962 & 0.0183343 \\
$\mathrm{v}, \mathrm{cm} / \mathrm{sec}$ & $10^{6}$ & $10^{6}$ & $10^{6}$ \\
\hline
\end{tabular}

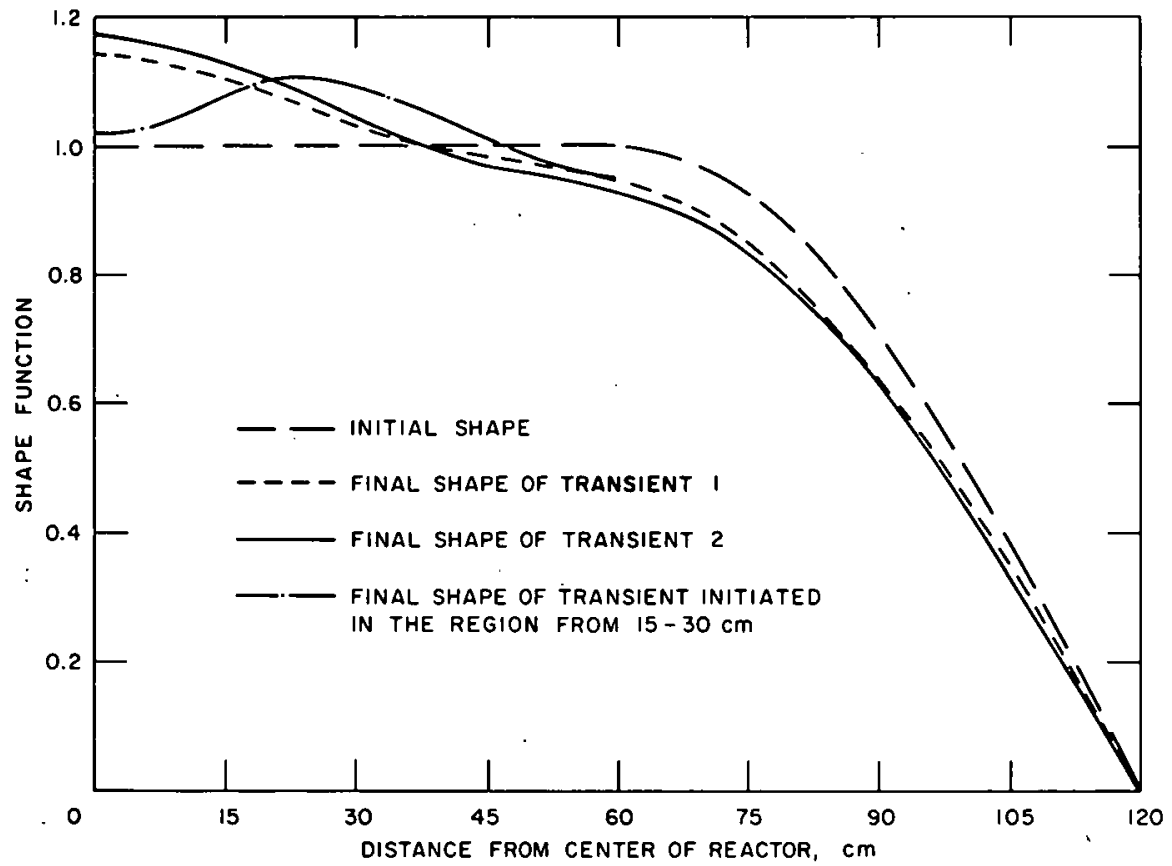

$113-1894$

Fig. 4. Shape Functions Used in Analysis of Transients

Two transients are analyzed. Both are initiated by a ramp change in the fission cross section in Region $1(0-15 \mathrm{~cm}$ from the origin) for $0.1 \mathrm{sec}$, which is then held

TABLE II. Region I Fission Cross Sections at End of Ramp

\begin{tabular}{ccc}
$\begin{array}{c}\text { Fission Cross } \\
\text { Section }\end{array}$ & Transient 1 & Transient 2 \\
\hline$\nu \Sigma_{\mathrm{f}}, \mathrm{cm}^{-1}$ & 0.0201461 & 0.0202962
\end{tabular}
constant for the remainder of the transient (up to $\mathrm{t}=$ l $\mathrm{sec}$ ). The final values of the fission cross section in Region 1 are given in Table II. One group of delayed neutrons is used, with $\beta=0.0064$, and $\lambda=$ $0.08 \mathrm{sec}^{-1}$. When the transients were analyzed with the "exact" finite-difference computer code WIGLE, ${ }^{2}$ it was found that Transient 1 was a subprompt critical burst, while Transient 2 was superprompt critical. The WIGLE solutions also yielded the final shapes shown in Fig. 4. 
To compare various choices of weighting functions, good sets of trial functions are used. The initial and final shapes for Transients 1 and 2 are two such sets. To study multichannel synthesis, though, a poor set of trial functions is chosen. One possibility is to choose the initial shape along with the final shape from a transient initiated in some other region of the reactor. One such choice is shown in Fig. 4. To obtain this shape, the fission cross section of the $15-30-\mathrm{cm}$ region was increased in a ramp until $t=0.1 \mathrm{sec}$, while the absorption cross section in the first region was also being increased. The transient was then continued until $t=1 \mathrm{sec}$ and then terminated. This set of shape functions is called the "poor" set of trial functions. This set is also used as weighting functions in the continuous-synthesis comparison.

A proper comparison of the space-time approximate methods should consider both amplitude and shape changes. One way of doing this is to form the following amplitude function at time t:

$$
P(t)=\frac{\int_{x} W(x, t) \bar{\phi}(x, t) d x}{\int_{x} W(x, 0) \bar{\phi}(x, 0) d x},
$$

where $\bar{\phi}(\mathrm{x}, \mathrm{t})$ is given by $\mathrm{Eq} .3 .3$, and $\mathrm{P}(\mathrm{t})$ is a scalar amplitude function characteristic of the entire reactor. For purposes of comparison with point kinetics, the choice

$$
W(x, t)=\bar{\phi}(x, 0)
$$

is made for each case treated. The shape functions at any time $t$ can then be determined by dividing $\phi(x, t)$ by $P(t)$ :

$$
\psi(\mathrm{x}, \mathrm{t})=\frac{\bar{\phi}(\mathrm{x}, \mathrm{t})}{\mathrm{P}(\mathrm{t})}
$$

Another useful parameter is the reciprocal period, given by

$$
\alpha(t)=\frac{1}{P(t)} \frac{d P}{d t} .
$$

Equation 8.4 can be integrated to obtain .

$$
P(t)=P(0) \exp \left[\int_{0}^{t} \alpha\left(t^{\prime}\right) d t^{\prime}\right]
$$

For the transients being analyzed in this report, the reciprocal period approaches an asymptotic value after the ramp change in fission cross section has terminated. This value can be determined by using Eq. 8.5 to obtain 


$$
\alpha_{\text {as }}=\frac{1}{t_{2}-t_{1}} \ln \frac{P\left(t_{2}\right)}{P\left(t_{1}\right)}, \quad t_{2}>t_{1}
$$

where $t_{1}$ and $t_{2}$ are times after which the reciprocal period has become asymptotic. These times can be found without difficulty by noting when the shape function has approached its asymptotic distribution.

The asymptotic reciprocal periods for each approximate method can be compared to see whether the error is bounded. This is the case only if the reciprocal period is (almost) identical to the exact value. If the error is bounded, then the ratio of the approximate value of $P\left(t_{2}\right)$ to the exact value gives an error measure.

\section{B. Continuous Synthesis}

The purpose of these studies is to compare different weightedresidual criteria in an attempt to discern the effect of weighting-function choice on accuracy. Both transients are analyzed to see if the condition of prompt criticality has any effect on the approximate methods. In the analysis of Transient 1 , the initial shape and the final shape for Transient 1 are chosen as trial functions. Since these "bracket" the true solution, accurate results are expected, and indeed are obtained, as is shown in Table III.

TABLE III. Transient 1 Results at $t=1$ sec, Using Initial and Final Shapes as Trial Functions

\begin{tabular}{|c|c|c|c|c|c|c|}
\hline & Exact & Galerkin & Subdomain 1 & Subdomain 2 & "Poor" & $\begin{array}{c}\text { Point } \\
\text { Kinetics }\end{array}$ \\
\hline Reciprocal period, $\sec ^{-1}$ & 1.91 & 1.91 & 1.90 & 1.92 & 1.91 & 0.49 \\
\hline Amplitude function & 102.90 & 102.85 & 102.59 & 103.90 & 102.20 & 10.87 \\
\hline Amplitude for mode 1 & - & 0.0056 & 0.0259 & -0.254 & 0.0324 & 10.87 \\
\hline Amplitude for mode 2 & - & 102.84 & 102.56 & 104.15 & 102.17 & - \\
\hline
\end{tabular}

The title Subdomain 1 refers to the choice of subdomains as unit step-functions defined as unity in the regions $0-15$ and $15-120 \mathrm{~cm}$ from the origin. Subdomain 2 uses $0-60$ and $60-120 \mathrm{~cm}$ as the subdomains. "Poor" weighting refers to the choice of the initial shape and the shape function characteristic of a transient initiated in the $15-30-\mathrm{cm}$ region of the reactor (see Fig. 4). The fact that the amplitude of mode 2 is dominant for all cases (except point kinetics) indicates that each approximation has also selected the proper shape at $t=1$ sec. This fact is also true for intermediate times, although the results are not tabulated here.

The poor results of point kinetics re-emphasize the purpose of space-time approximations. Although the shape change (or "flux tilt") shown in Fig. 4 does not appear to be substantial, it has a profound effect 
on the reciprocal period and hence the amplitude function, as Table III shows. Transient 1 , or one similar to it, could very well be initiated in an actual reactor. It would seem from point kinetics that the transient is not too severe. Actually, it is nearly a prompt critical burst ( $\rho=97.5$ cents). Point kinetics calls it an 86.0-cent burst. A slightly higher increase in $\nu \Sigma_{f}$ would have caused a superprompt critical burst, which point kinetics could not have predicted.

Table IV and Fig. 5. contain the results from choosing the "poor" shapes as trial functions. When these functions are also used as the weighting

TABLE IV. Transient 1 Results at $t=1 \mathrm{sec}$, Using "Poor" Trial Functions

\begin{tabular}{|c|c|c|c|c|}
\hline & Exact & $\begin{array}{l}\text { Transient } \\
\text { Weighting }\end{array}$ & Galerkin & $\begin{array}{c}\text { Point } \\
\text { Kinetics }\end{array}$ \\
\hline Reciprocal period, $\sec ^{-1}$ & 1.91 & 1.89 & 0.52 & 0.49 \\
\hline Amplitude function & 102.90 & 100.34 & 11.60 & 10.87 \\
\hline Amplitude for mode 1 & - & -216.44 & 9.51 & 10.87 \\
\hline Amplitude for mode 2 & - & 316.78 & 2.08 & - \\
\hline
\end{tabular}

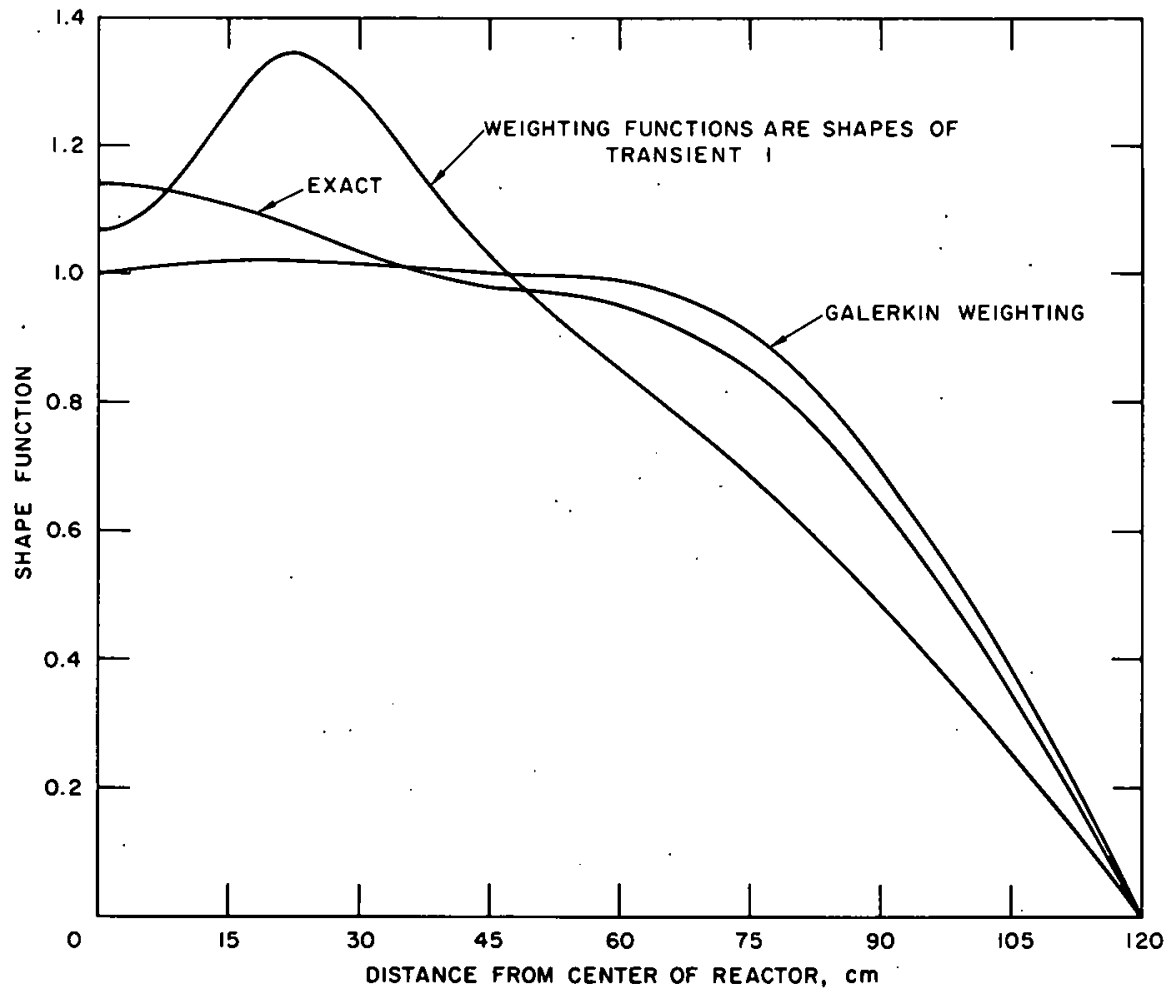

$113-1893$

Fig. 5. Transient 1 Shape Functions at $t=1 \mathrm{sec}$, Using "Poor" Trial Functions 
functions, that is, when Galerkin weighting is used, very little improvement is made over point kinetics. If, on the other hand, the true shapes of $\operatorname{Tran}$ sient 1 happen to be chosen as the weighting functions, the reciprocal period and the amplitude function come very close to the exact values. However, the resulting shape function is incorrect, as can be seen in Fig. 5. These results can be explained in terms of the variational method described in Chapter III.

The flux and the adjoint flux both appear in each term of the functional given by Eq. 3.45. The functional is stationary, so that its first variation with respect to either $\phi$ or $\phi^{*}$ vanishes. In either case, approximate solutions (such as those given in Eqs. 3.3 and 3.46, where $\psi_{j}(\mathrm{x})$ and $\psi_{i}^{*}(\mathrm{x})$ are trial functions) for both $\phi$ and $\phi^{*}$ must be used to determine the value of the functions. Furthermore, the accuracy of the value of the functional depends upon how well each set of trial functions is chosen. This argument can be extended to any weighted-residual method because the variational method is really two weighted-residual methods, in which the trial functions for the real problem are the weighting functions for the adjoint problem, and vice versa.

Taking the first variation also provides a means of determining the undetermined amplitude functions for each problem. If either set of trial functions is "good," both sets of amplitude functions will be determined fairly accurately. If both sets of trial functions are good, the amplitude functions are determined even more accurately. If both sets are "poor," neither set of amplitude functions is determined accurately. On the other hand, the shape of the true flux is poor if poor flux trial functions are chosen, no matter how good the weighting functions are. One conclusion that can be drawn from the above results and discussion is that, if a good set of trial functions is chosen for the flux, Galerkin weighting always gives an accurate solution for the flux and for the reciprocal period. In this sense, Galerkin weighting is good strategy.

When the initial and final shapes of Transient 2 were chosen as trial functions for Transient 2, results similar to those obtained in the analysis of Transient 1 were obtained. These are shown in Table V. Notice that Galerkin weighting is nearly exact. Table VI contains the results when the shape functions from Transient $l$ are chosen as trial functions to analyze

TABLE V. Transient 2 Results at $t=1$ sec, Using Initial and Final Shapes as Trial Functions

\begin{tabular}{lcccccc}
\hline & Exact & Galerkin & Subdomain 1 & Subdomain 2 & "Poor" & $\begin{array}{c}\text { Point } \\
\text { Kinetics }\end{array}$ \\
\hline Reciprocal period, sec ${ }^{-1}$ & 29.51 & 29.51 & 29.71 & 29.44 & 29.52 & 8.84 \\
Amplitude function & $3.94 \times 10^{12}$ & $3.93 \times 10^{12}$ & $4.72 \times 10^{12}$ & $3.70 \times 10^{12}$ & $3.92 \times 10^{12}$ & $4.87 \times 10^{4}$ \\
Amplitude for mode 1 & - & $3.86 \times 10^{8}$ & $-3.08 \times 10^{10}$ & $5.56 \times 10^{9}$ & $-7.69 \times 10^{8}$ & $4.87 \times 10^{4}$ \\
Amplitude for mode 2 & - & $3.93 \times 10^{12}$ & $4.75 \times 10^{12}$ & $3.69 \times 10^{12}$ & $3.93 \times 10^{12}$ & - \\
\hline
\end{tabular}


TABLE VI. Transient 2 Results at $t=1 \mathrm{sec}$, Using Transient 1 Shapes as Trial Functions

\begin{tabular}{lcccccc}
\hline & Exact & Galerkin & Subdomain 1 & Subdomain 2 & "Poor" & $\begin{array}{c}\text { Point } \\
\text { Kinetics }\end{array}$ \\
\hline Reciprocal period, $\mathrm{sec}^{-1}$ & 29.51 & 29.48 & 29.82 & 29.36 & 28.99 & 8.84 \\
Amplitude function & $3.94 \times 10^{12}$ & $3.82 \times 10^{12}$ & $5.24 \times 10^{12}$ & $3.43 \times 10^{12}$ & $2.45 \times 10^{12}$ & $4.87 \times 10^{4}$ \\
Amplitude for mode 1 & - & $-9.56 \times 10^{11}$ & $-1.35 \times 10^{12}$ & $-8.24 \times 10^{11}$ & $-5.45 \times 10^{11}$ & $4.87 \times 10^{4}$ \\
Amplitude for mode 2 & - & $4.78 \times 10^{12}$ & $6.59 \times 10^{12}$ & $4.25 \times 10^{12}$ & $3.00 \times 10^{12}$ & - \\
\hline
\end{tabular}

Transient 2. The Galerkin method is clearly superior to the other methods in this case. In addition, "poor" weighting does not do as well as before. Note that amplitude 1 is negative and has a substantial magnitude in all cases. However; when the solution is formed for each approximation using Eq. 3.3, and when Eq. 8.3 is used, each approximation gives nearly the correct flux shape. Figure 6 shows the total flux at $t=1$ sec for each approximation. Subdomain l weighting overestimates the total flux, although each gives nearly the correct flux shape. Because of this, it may be concluded that, when the trial functions become "less good," a good choice of weighting functions becomes more important in obtaining an accurate solution. Furthermore, the best possible choice of subdomains is usually not clear, indicating that Galerkin weighting is sounder strategy than subdomain weighting.

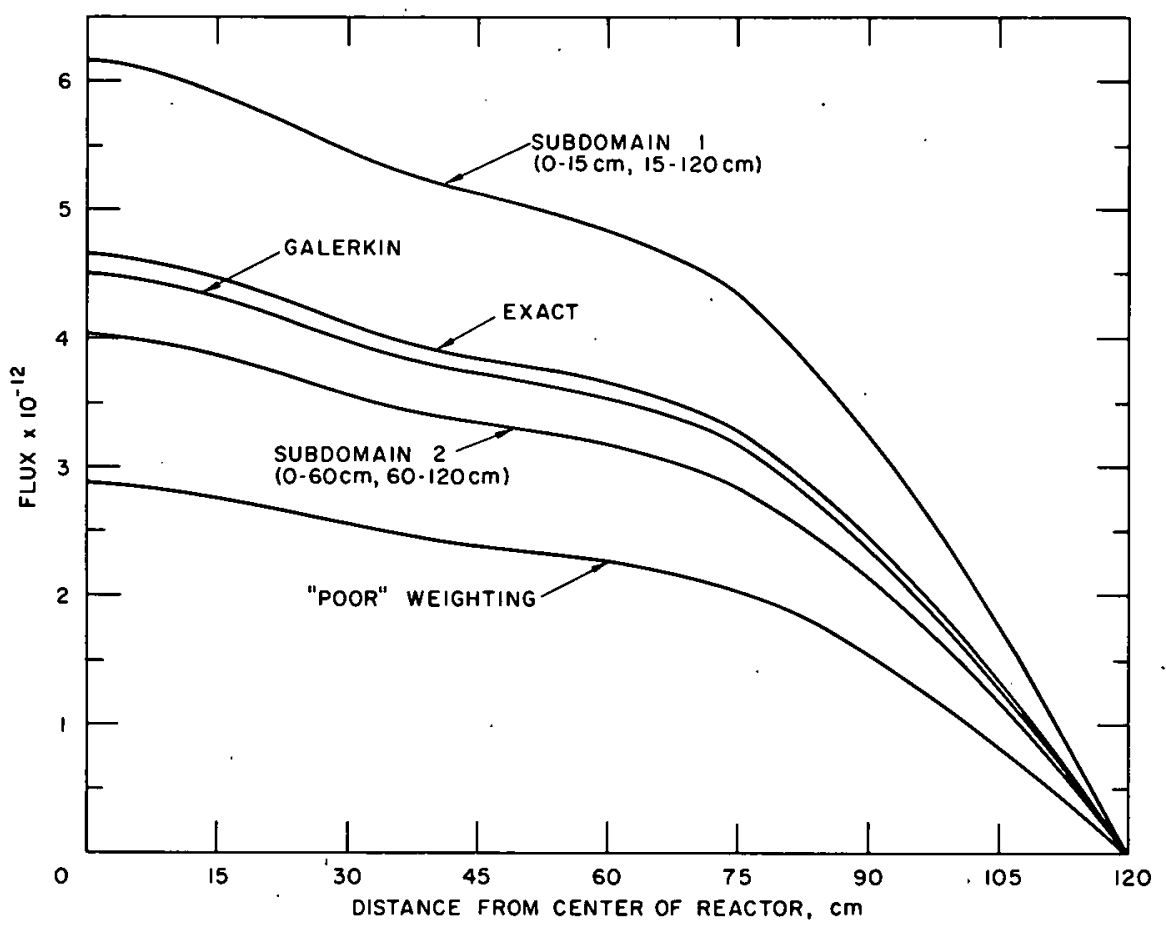

$113-1892$

Fig. 6. Transient 2 Flux Distribution at $\mathrm{t}=1 \mathrm{sec}$, Using Transient 1 Shapes as Trial Functions 
C. Multichannel Synthesis

1. Nodal Approximations

Studies of the nodal approximations are undertaken here for two reasons: to determine the degree of improvement as the number of

TABLE VU. Interface Locations for Multichannel Approximations

Number of Channels Interface Locations, $\mathrm{cm}$ from Origin

\begin{tabular}{rrrrrrrrrr}
\hline 2 & 15 & & & & & & \\
3 & 15 & 30 & & & & & \\
4 & 15 & 30 & & 60 & & & \\
5 & & 15 & 30 & & 60 & & 90 & \\
8 & 15 & & 30 & 45 & 60 & 75 & 90 & 105 \\
10 & 7 & 15 & 22 & 30 & 45 & 60 & 75 & 90 & 105 \\
\hline
\end{tabular}
nodes is increased, and to compare partial-current and surfacecancellation weighting of the interface conditions. Galerkin weighting is applied to the equation residuals. Table VII lists the interfaces chosen for the various nodal approximations. Tables VIII and IX compare the reciprocal periods and amplitude functions for Transients 1 and 2, respectively.

Partial-current weighting does much better in all cases. Indeed, five-node partial-current weighting is more accurate than ten-node surfacecancellation weighting. Figure 7 shows the shape functions of Transient 2 at $t=1 \mathrm{sec}$ for the ten-node partial-current and surface-cancellation weightings. The partial-current shape is much closer to the true shape than is the surface-cancellation shape. The jump discontinuities appear at the nodal interfaces as a natural consequence of the use of nodal methods. A similar set of curves can also be drawn for Transient 1.

TABLE VIII. Transient 1 Results at $t=1 \mathrm{sec}$ for Nodal Approximations

\begin{tabular}{lccccr}
\hline & \multicolumn{2}{c}{ Reciprocal Period, sec $^{-1}$} & & \multicolumn{2}{c}{ Amplitude Function } \\
\cline { 2 - 3 } & $\begin{array}{c}\text { Surface } \\
\text { Cancellation }\end{array}$ & $\begin{array}{c}\text { Partial } \\
\text { Current }\end{array}$ & & $\begin{array}{c}\text { Surface } \\
\text { Cancellation }\end{array}$ & $\begin{array}{c}\text { Partial } \\
\text { Current }\end{array}$ \\
\hline Exact & 1.91 & 1.91 & & 102.90 & 102.90 \\
Point kinetics & 0.49 & 0.49 & & 10.87 & 10.87 \\
Two nodes & 0.52 & 0.60 & & 11.54 & 14.17 \\
Five nodes & 0.56 & 0.80 & & 12.77 & 21.37 \\
Eight nodes & 0.60 & 1.04 & & 14.23 & 32.99 \\
Ten nodes & 0.64 & 1.58 & 15.43 & 70.06 \\
\hline
\end{tabular}

TABLE IX. Transient 2 Results at $t=1 \mathrm{sec}$ for Nodal Approximations

\begin{tabular}{lccccc}
\hline & \multicolumn{2}{c}{ Reciprocal Period, sec } & & \multicolumn{2}{c}{ Amplitude Function } \\
\cline { 5 - 6 } & $\begin{array}{c}\text { Surface } \\
\text { Cancellation }\end{array}$ & $\begin{array}{c}\text { Partial } \\
\text { Current }\end{array}$ & & $\begin{array}{c}\text { Surface } \\
\text { Cancellation }\end{array}$ & $\begin{array}{c}\text { Partial } \\
\text { Current }\end{array}$ \\
\hline Exact & 29.51 & 29.51 & & $3.94 \times 10^{12}$ & $3.94 \times 10^{12}$ \\
Point kinetics & 8.84 & 8.84 & & $4.87 \times 10^{4}$ & $4.87 \times 10^{4}$ \\
Two nodes & 9.83 & 12.99 & & $1.13 \times 10^{5}$ & $1.71 \times 10^{6}$ \\
Five nodes & 11.28 & 17.87 & & $3.93 \times 10^{5}$ & $1.23 \times 10^{8}$ \\
Eight nodes & 12.67 & 21.55 & & $1.29 \times 10^{6}$ & $3.19 \times 10^{9}$ \\
Ten nodes & 13.78 & 26.70 & & $3.42 \times 10^{6}$ & $3.17 \times 10^{11}$ \\
\hline
\end{tabular}




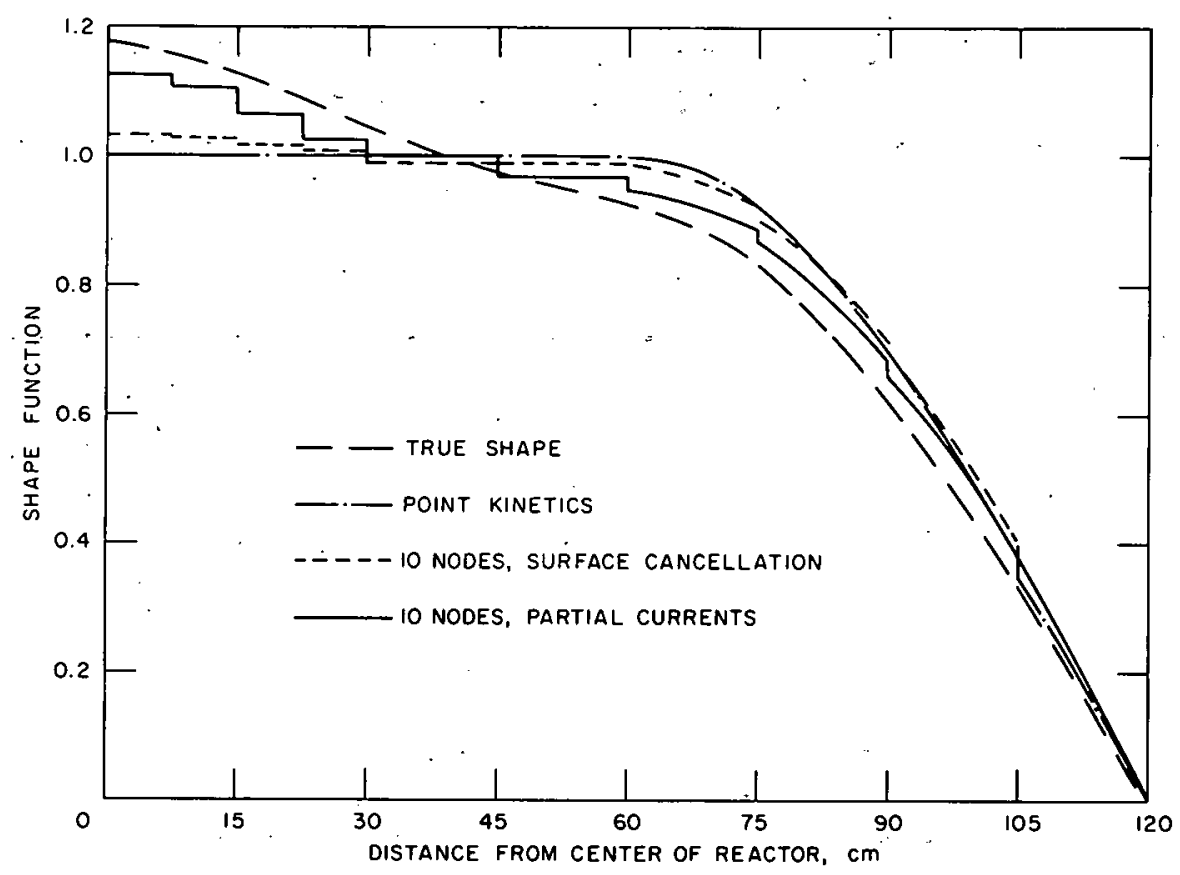

$113-1890$

Fig. 7. Transient 2 Shape Functions at $t=1 \mathrm{sec}$ for 10 Nodes

Surface-cancellation weighting is a very poor choice for nodal methods, whereas partial-current weighting does quite well. However, when a good set of trial functions is used, nodal methods in general seem to be inferior to the synthesis approach. One possible explanation for the relative success of partial-current weighting is that, in diffusion theory, the neutron leakage from one region to another is given by the partial current at the region interface. On purely mathematical grounds, however; there seems to be no clear reason for preferring one type of weighting over the other. One important conclusion can be drawn from these studies: The manner of weighting the interface residuals is crucial to the success of multichannel synthesis. The entire area requires much more study for clarification.

\section{Two Modes in Each Channel}

The main purpose of the two-mode multichannel synthesis studies is to determine how well multichannel synthesis can do when a "poor" choice of trial functions is made. The trial functions are (1) the initial shape, and (2) the final shape for the transient initiated in the region $15-30 \mathrm{~cm}$ from the origin (see Fig. 4). Galerkin weighting is applied to the differential equation and partial-current weighting is used on the interface residuals. Tables $X$ and $X I$ list the reciprocal periods and amplitude functions for each transient.. Table VII gives the interface locations. Note. that single-channel synthesis is little better than point kinetics. 
TABLE X. Transient 1 Results at $. t=1$ sec. for Multichannel Synthesis

\begin{tabular}{lcc}
\hline & $\begin{array}{c}\text { Reciprocal } \\
\text { Period, } \\
\text { sec }^{-1}\end{array}$ & $\begin{array}{c}\text { Amplitude } \\
\text { Function }\end{array}$ \\
\hline Exact & 1.91 & 102.90 \\
One channel & 0.52 & 11.60 \\
Two channels & 0.93 & 26.88 \\
Three channels & 1.24 & 44.15 \\
Four channels & 1.24 & 44.23 \\
\hline
\end{tabular}

TABLE XI. Transient 2 Results at $t=1 \mathrm{sec}$ for Multichannel Synthesis

\begin{tabular}{lcl}
\hline & $\begin{array}{c}\text { Reciprocal } \\
\text { Period, } \\
\text { sec }^{-1}\end{array}$ & $\begin{array}{c}\text { Amplitude } \\
\text { Function }\end{array}$ \\
\hline Exact & 29.51 & $3.94 \times 10^{12}$ \\
One channel & 9.92 & $1.24 \times 10^{5}$ \\
Two channels & 20.51 & $1.26 \times 10^{9}$ \\
Three channels & 24.56 & $4.64 \times 10^{10}$ \\
Four channels & 24.58 & $4.72 \times 10^{10}$
\end{tabular}

There is virtually no improvement for either transient when the number of channels is increased from three to four. This is because the fourth channel was added where it was not really needed. If three channels had been placed in the inner $30 \mathrm{~cm}$, a four-channel approximation would probably have done much better. In practice, however, it seems advisable to choose channels in a symmetric manner in order to be able to handle nonlinear transients which can cause drastic shape changes.

The greatest improvement is shown when the number of channels is increased from one to two. This fact is even more apparent when the shape functions, shown in Fig. 8, are considered. The shape function for one channel is completely incorrect and is close to point kinetics. It is much better for two channels, and better still for three channels. The shape function for four channels is identical to that for three channels. The discontinuities arise naturally at the interfaces between channels due to the nature of the approximation. Figure 8 is for Transient 2, but a similar result holds for Transient 1 . 


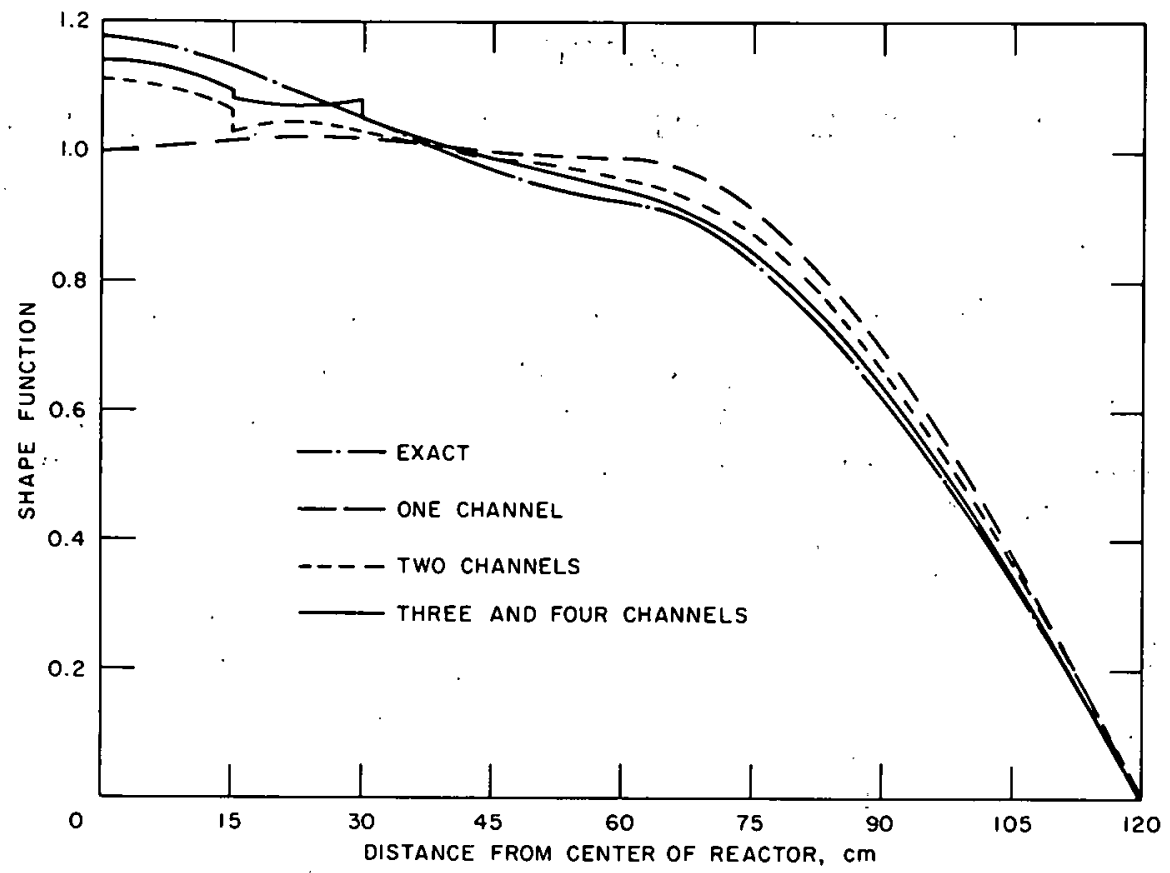

$113-1891$

Fig. 8. Transient 2 Shape Functions at $t=1 \mathrm{sec}$ for Multichannel Synthesis

The above discussion indicates that multichannel synthes is is capable of yielding a reasonable approximation even with a poor choice of trial functions. Some care should be taken, however, in choosing the channel interfaces. Furthermore, care should be taken in the choice of the method of weighting the interfaces. 


\section{CHAPTER IX}

\section{CONCLUSIONS AND RECOMMENDATIONS FOR FURTHER STUDY}

\section{A. Conclusions}

The point-reactor model, modal-expansion methods, synthesis methods, variational methods, multichannel-synthesis methods, temporally discontinuous methods, and quasistatic methods of solving the timedependent neutron-diffusion equations can all be thought of as weightedresidual methods. They differ only in the manner in which their trial functions are chosen. The multimode kinetics equations can be used for the time integration in each method. For self-adjoint systems, the varia. tional method and the Galerkin method are identical. For non-self-adjoint systems, the variational method is equivalent to solving both the real problem and the adjoint problem simultaneously using a weighted-residual method for each. The trial functions for one are the weighting functions for the other.

There does not seem to be a need for seeking variational principles for nonlinear systems. They are difficult to find, and do not yield much more information than the Galerkin method. Therefore, a weighted-residual procedure should be adopted from the outset.

When spatially discontinuous trial-function schemes are adopted, the interface conditions must be treated by a weighted-residual method. Partial.. current weighting seems to be a better choice than surface-cancellation weighting in this respect. Many other possibilities could have been investigated, however. For these, the weighting functions used at the interfaces should be related to the functions used to weight the differential equations. A linear combination of weighting functions for each channel, evaluated at the interfaces, would be a natural choice.

Temporally discontinuous trial-function methods are suitable for analyzing nonlinear transients. If the trial functions are precalculated, the "new initial residuals" must be weighted whenever new trial functions are introduced and/or old trial functions are removed. A good choice of weighting functions is the set of weighting functions to be used throughout the ensuing time interval. When trial functions are recalculated, as in a quasistatic approximation, iteration schemes for shape-function recalculation are generally used. In these cases, the "new initial residuals" vanish identically, since the correct shape function for the beginning of the ensuing time interval has been found.

The shape function for the first pass through a time interval in an iterative quasistatic scheme can be improved if a nodal method is used instead of assuming a constant shape. This will enable the time interval to 
be extended, so that fewer shape recalculations will be needed to analyze a transient. One such nodal method has been applied in this manner.

The multimode kinetics equations can be integrated using the method of undetermined parameters. The finite-difference time-step limitation is overcome when this method is used. Piecewise polynomial functions are good choices for trial functions.

Space-time iteration using the method of undetermined functions provides a means of overcoming the obstacle of having to guess at trial functions, because it finds a solution that is independent of the initial choice of trial functions. It can be a powerful tool in multidimensional studies.

For continuous synthesis, Galerkin weighting will always give accurate solutions when a good set of trial functions is chosen. Partial-current weighting was superior to surface-cancellation weighting of interface conditions when multichannel synthesis was used. Multichannel synthesis is an improvement over single-channel synthesis when the choice of trial functions is poor.

In conclusion, weighted-residual methods can be highly successful when applied to problems of space-dependent reactor dynamics. Some of these methods are intrinsically better than others. For example, the discontinuous-trial-function methods offer greater flexibility than continuous-trial-function methods. Furthermore, the traditional pointreactor model has the least flexibility, since only one trial function is used throughout. In each of the methods, however, the degree of success depends strongly on the ability to choose good trial functions:

B. Recommendations for Further Study

The proper role of interface conditions in multichannel synthesis needs to be much better understood. A sound method of weighting the interface residuals is needed.

The space-time iteration scheme should be pursued, because it is potentially very useful for analyzing higher dimensional systems. Its use with temporally discontinuous-trial-function methods also appears to be promising.

Finally, a topic that was judged to be outside the scope of this report should be studied. This is the area of finding error bounds for trial-function methods. When precalculated trial functions are used, there is no way of knowing how accurate the solution is unless it is compared with the exact solution. If this must be done too often, the utility of the approximation method would be destroyed. 
Linear Dependence

Consider a multichannel synthesis approximation in which two modes are chosen for each synthesis region. Although the trial functions may be selected so that they are linearly independent over the entire reactor, they could be linearly dependent, or "almost" linearly dependent, within a channel. ${ }^{66}$ Suppose that the trial functions within a channel are given by $\psi_{1}(\mathbf{x})$ and $\psi_{2}(\mathbf{x})$. To determine the degree of linear dependence between $\psi_{1}(\mathrm{x})$ and $\psi_{2}(\mathrm{x})$, the normalized Gram determinant ${ }^{67}$ of their inner products is formed. The procedure is outlined here.

The inner products are given by

$$
G_{i j}=\int_{x} \psi_{i}(x) \psi_{j}(x) d x, \quad i, j=1,2 .
$$

The Gram matrix $G$ is given by

$$
G=\left[\begin{array}{ll}
G_{11} & G_{12} \\
G_{21} & G_{22}
\end{array}\right]
$$

Now, define a diagonal matrix $\mathrm{D}$ by

$$
D=\left[\begin{array}{ll}
G_{11}^{-1 / 2} & 0 \\
0 & G_{22}^{-1 / 2}
\end{array}\right]
$$

The normalized Gram matrix is given by

$$
G^{\prime}=D G D=\left[\begin{array}{cc}
1 & G_{12}\left(G_{11} G_{22}\right)^{-i / 2} \\
G_{21}\left(G_{11} G_{22}\right)^{-1 / 2} & 1
\end{array}\right] .
$$

Finally, the normalized Gram determinant is

$$
\operatorname{det}\left(G^{\prime}\right)=1-\frac{G_{12} G_{21}}{G_{11} G_{22}} .
$$

In principle, $\psi_{1}(x)$ and $\psi_{2}(x)$ are linearly dependent if and only if $\operatorname{det}\left(G^{\prime}\right)=0$. Numerically, however, they may be "almost" linearly dependent if $\operatorname{det}\left(G^{\prime}\right)$ is very small. Therefore, a criterion for defining "almost" linear dependence must be defined. One such criterion ${ }^{66}$ is

$$
\operatorname{det}\left(G^{\prime}\right)<\frac{\epsilon}{2}
$$


where $\epsilon$ is a small positive number chosen beforehand. If Inequality A.6 is satisfied, two possibilities exist. Either one of the trial functions should be discarded, or the locations of the interfaces should be changed.

The same procedure must be repeated for the weighting functions within the channel. They must be linearly independent as well, if a solution is to be obtained. If Galerkin weighting is used, however, a separate comparison for the weighting functions is not necessary.

Linear dependence can also occur in the integration of the multimode kinetics equations. The system of equations given by Eq. 6.36 can be written in the matrix shorthand form

$$
L A=R \text {, }
$$

where $A$ and $R$ are IK-dimensional column vectors and $L$ is an IK $x$ IK matrix. If

$$
\operatorname{det}(L)=0
$$

the matrix $L$ is singular and the system is linearly dependent. 
APPENDIX B

Selection of Time-step Size

When the method of undetermined parameters is used to solve the multimode kinetics equations, methods that regulate the size of the time intervals can be incorporated. Such methods allow large time steps to be taken when the transient is proceeding slowly, and automatically shorten the time step when more rapid changes occur. They also ensure a stable solution for every time step taken. One such method is the time-step halving technique, ${ }^{11}$ which is described here.

Consider the time step

$$
\Delta t_{i}=t_{i+1}-t_{i}, \because
$$

where the solution to the multimode kinetics equations is known at $t_{i}$, and desired at $t_{i+1}$. Two independent solutions to the multimode kinetics equations are now found. The first of these integrates over the entire time step, yielding a solution at $t=t_{i+i}$. The second solution is for only half the step, yielding a solution at

$$
t=t_{i}+\frac{\Delta t_{i}}{2}
$$

Each solution is found by determining a different set of parameters. The half-step solution is expressed as a different polynomial for each mode. Suppose each of these polynomials is extrapolated to obtain values at $t=t_{i+i}$. These values will not be the same as those found by integration over the entire time step, but they should be close. The degree of closeness of these values determines the next step.

To compare the two sets of values, form the following error norm:

$$
\epsilon=\left[\frac{\sum_{k=1}^{K} N_{k, e x t}^{2}-N_{k}^{2}\left(t_{i+1}\right)}{\sum_{k=1}^{K} N_{k, e x t}^{2}}\right]^{1 / 2},
$$

where $K$ is the number of modes, $N_{k \text {, ext }}$ is the value of the $k$ th amplitude function calculated for the half-step and extrapolated to the end of the step, and $N_{k}\left(t_{i+l}\right)$ is the value of the kth amplitude function from the full-step calculation. The error norm is now compared with a preselected tolerable error $\epsilon_{1}$. If $\epsilon<\epsilon_{l}$, then the amplitude functions $N_{k}\left(t_{i+1}\right), k=1, \ldots, K$, are acceptable as the solutions, and the multimode kinetics equations can be 
integrated over the next time step. If, in addition, $\epsilon<C \epsilon_{1}$, where $C$ is a preselected parameter less than unity, the size of the next time step is doubled. A good value for $C$ is 0.1 .

If, on the other hand, $\epsilon>\epsilon_{1}$, the values $\mathrm{N}_{k}\left(t_{i+1}\right)$ are not acceptable, and the time step is halved. Two independent solutions to the multimode kinetics equations are again found, this time for the times $t=t_{i}+\Delta t_{i} / 2$, and $t=t_{i}+\Delta t_{i} / 4$. Note that the first of these has already been made for the original comparison, so that it does not have to be repeated. The solutions are again compared as described above: If acceptable values for $\mathrm{N}_{k}\left(\mathrm{t}_{\mathrm{i}}+\Delta \mathrm{t}_{\mathrm{i}} / 2\right)$ are found, the solution at $\mathrm{t}=\mathrm{t}_{\mathrm{i}+1}$ will again be attempted in the same manner. This time, however, $t=t_{i}+\Delta t_{i} / 2$ is the lower end of the time step, so that independent solutions are formed for $t=t_{i}+(3 / 4) \Delta t_{i}$; and $t=t_{i+1}$.

If the values $N_{k}\left(t_{i}+\Delta t_{i} / 2\right)$ are not acceptable, the time step is halved again and the calculation is repeated for the quarter-step. The method continues until an acceptable solution has been found for $t=t_{i+1}$. The procedure then begins anew to determine the $\mathrm{N}_{k}\left(t_{i+2}\right)$, etc., until the end of the transient is reached. 


\section{ACKNOW LEDGMENTS}

I would like to thank Dr. David L. Hetrick for his invaluable guidance and encouragement, not only during the period of this work, but also throughout my tenure as a graduate student. I owe a great debt of gratitude to Dr. Daniel A. Meneley of Argonne National Laboratory. His invaluable advice and criticism enabled this work to be completed.

I am grateful to the Argonne Universities Association for supporting this work with an AUA Predoctoral Fellowship, and to the Reactor Physics Division of Argonne National Laboratory for providing its facilities so that I could perform the research.

I wish to thank Dr. Victor Luco of Argonne National Laboratory for many hours of helpful discussion. 


\section{REFERENCES}

1. S. Kaplan, A。F。 Henry, S。G。 Margolis, and J。 J. Taylor, "Space-, Time Reactor Dynamics," Third United Nations International Conference on the Peaceful Uses of Atomic Energy 3, 41 (1964).

2. W. R. Cadwe11, A. F. Henry, and A. J。 Vigilotti, WIGLE - A Program for the Solution of the Two-Group Space-Time Diffusion Equations in Slab Geometry, WAPD-TM-416 (1964).

3. D. L. Hetrick, Dynamics of Nuclear Reactors, book manuscript in preparation (1969).

4. A. F. Henry and A. V. Vota, WIGL2 - A Program for the Solution of the One-Dimensional, Two-Group, Space-Time Diffusion Equations Accounting for Temperature, Xenon, and Controi Feedback, WAPD-TM-532 (1965).

5. James W. Smiley, STREAK - A Numerical Solution for Space-Time Neutron Diffusion Equations, Engineering Research Bulletin B-95, The Pennsylvania State University, University Park, Pennsylvania (1966).

6. T. A. Porsching, Numerical Solution of the Reactor Kinetics Equations by Approximate Exponentials, Nuc1。Sci。Eng。25, 183 (1966).

7. K. F. Hansen, B. V. Koen, and W. Wo Little, Jr., Stable Numerical Solutions of the Reactor Kinetics Equations, Nucl. Sci. Eng. 22, 51 (1965)。

8. J. Barclay Andrews II and K. F. Hansen, Numerical Solution of the TimeDependent Multigroup Diffusion Equations, Nucl. Sci. Eng. 31, 304 (1968).

9. F. T. Adler, Reactor Kinetics: Integral Equation Formulation, Reactor Sci. Technol。(J. Nucl。 Energy, Parts A/B) 15, 81 (1961).

10. R。 O. Brittan, Some Problems in the Safety of Fast Reactors, ANL-5577 (1956).

11. J. J. Kaganove, Numerical Solution of the One-group Space-independent Reactor Kinetics Equations for Neutron Density Given the Excess Reactivity, ANL-6132 (Feb 1960)。

12. John Szeligowski and David Hetrick, "A Comparison of Some Numerical Methods for Solving the Equations of Reactor Dynamics," Proceedings of the Intermational Conference on the Utilization of Research Reactors, and Reactor Mathematics and Computation, Mexico City, II, 994 (1967).

13. Ao Fo Henry, The Application of Reactor Kinetics to the Analysis of Experiments, Nucl。'Sci. Eng。3, 52 (1958).

14. A. F。 Henry and No Jo Curlee, Verification of a Method of Treating Neutron Space-Time Problems, Nucl。Sci.Eng。4, 727 (1958).

15. K. 0. Ott and D. A. Meneley, Accuracy of the Quasistatic Treatment of Spatial Reactor Kinetics, Nucl. Sci. Eng. 36, 402 (1969).

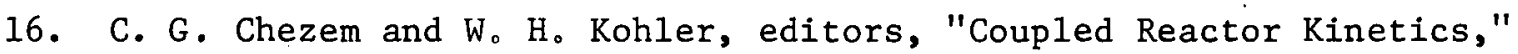
Proceedings of the National Topical Meeting at Texas A\&M University, The Texas A\&M Press (1967)。

17. H. L. Garabedian and G。 B。 Leffert, A Time-Dependent Analysis of Spatial" Flux Distributions, Nuc1. Sci. Eng. 6, 26 (1959). 
18. S. Kaplan, 0. J. Marlowe, and J. Bewick, Application of Synthesis Techniques to Problems Involving Time Dependence, Nucl. Sci. Eng. 18, 163 (1964).

19. D. E。 Dougherty and C. No Shen, The Space-Time Neutron Kinetic Equations Obtained by the Semidirect Variational Method, Nucl. Sci. Eng. 13; 141 (1962)。

20. S. H. Cranda11, Engineering Analysis, McGraw-Hill, New York, New York (1956)。

21. S. Kaplan, Some New Methods of Flux Synthesis, Nucl. Sci. Eng. 13, 22 (1962).

22. W. F. Ames, Nonlinear Partial Differential Equations in Engineering, Academic Press, New York (1965)。

23. S. Kaplan, "Synthesis Methods in Reactor Analysis," Advances in Nuclear Science and Technology 3, 233, edited by Paul Greebler and Ernest J。Henley, Academic Press, New York (1966).

24. W. C. Clarke and S. G. Margolis, The Multimode Synthesis Approximation for Space-Time Reactor Dynamics: An Appraisal of Finite Differencing Methods, WAPD-TM-635 (1967).

25. D. S. Selengut, "Variational Analysis of Multi-dimensional Systems," Hanford Quarterly Report, HW-59126, 89 (1959).

26. Jeffery Lewins, Importance: The Adjoint Function, Pergamon Press, Oxford (1965)。

27. E. L. Wachspress and M。 Becker, "Variational Synthesis with Discontinuous Trial Functions," Proceedings of the Conference on the Application of Computing Methods to Reactor Problems, May 17-19, 1965, Margaret Butler, General Chairman, ANL-7050, pp。191-205 (Aug 1965).

28. A. Jo Buslik, Interface Conditions for Few-Group Neutron Diffusion Equations with Flux-Adjoint Weighted Constants, Nucl. Sci. Eng. 32, 233 (1968).

29. J. B. Yasinsky, The Solution of the Space-Time Neutron Group Diffusion Equations by a Time-Discontinuous Synthesis Method, Nucl. Sci. Eng. 29, 381 (1967)。

30. Weston M: Stacey, Jr., Variational Functionals for Space-Time Neutronics, Nucl. Sci. Eng. 30, 448 (1967)。

31. Garret Birkhoff and Carl R。 DeBoor, "Piecewise Polynomial Interpolation. and Approximation," Approximation of Functions, edited by Henry L. Garabedian, Elsevier Publishing Co., Amsterdam, p. 164 (1965).

32. Arnold D. Kerr, An Extension of the Kontorovich Method, Quarterly of Applied Math。XXVI, 219 (1968).

33. B. A. Finlayson and L。 E. Scriven, The Method of Weighted Residuals A Review, App1. Math. Rev. 19, 735 (1966)。

34. L. V. Kantorovich and V. I, Krylov, Approximate Methods of Higher Analysis, Wiley (Interscience), New York (1959).

35. Wo G. Bickley, Experiments in Approximating to the Solution of a Partial Differential Equation, Phil. Mag. (7) 32, 50 (1941). 
36. Walter Zane Collings, The Method of Undetermined Functions as Applied to Approximating Solutions of Linear and Non-Linear, Transient Heat Conduction Equations, M.M.E. Thesis, University of Delaware (1961).

37. G. P. Calame and F。 D. Federighi, A Variational Procedure for Determining Spatially Dependent Thermal Spectra, Nucl. Sci. Eng. 10, 190 (1961)。

38. W. J. Duncan, Note on Galerkin's Method for the Treatment of Problems Concerming Elastic Bodies, Phil. Mag. (7) 25, 628 (1938).

39. Martin Becker, The Principles and Applications of Variational Methods, M.I.T. Press, Cambridge, Massachusetts (1964).

40. B. A. Finlayson and L. E. Scriven, On the Search for Variational Principles, Int. J. Heat Mass Transfer 10, 799 (1967).

41. Bruce Alan Finlayson, Approximate Solutions of Equations of Change; Convective Instability by Active Stress, Ph.D. Thesis, University of Minnesota (1965)。

42. S. G. Mikhlin, Variational Methods in Mathematical Physics, Macmillan, New York (1964)。

43. J. W. Green, An Expansion Method for Parabolic Partial Differential Equations, J. Res. Nat1。Bur. Std。51, 127 (1953).

44. Mocker, A.Generalized Formulation of Point Nuclear Reactor Kinetics Equations, Nucl。Sci.Eng。31, 458 (1968).

45. H. Soodak, "Problems of Reactor Kinetics," Proceedings of Symposia in Applied Mathematics, Vol. XI, edited by G. Birkhoff and

E. P. Wigner, American Mathematical Society, 233-255 (1961).

46. T. Gozani, The Concept of Reactivity and Its Application to Kinetic Measurements, Nukleonik 5, 55 (1963)。

47: E. E. Gross and J. H. Marable, Static and Dynamic MuItiplication Factors and Their Relation to the Inhour Equation, Nucl. Sci. Eng. ?, 281 (1960).

48. T. A. Porsching, On the Spectrum of a Matrix Arising from a Problem in Reactor Kinetics, SIAM J. Appl。 Math. 16, 301 (1968).

49. R. V. Churchill, Fourier Series and Boundary Value Problems, McGrawHil1, New York (1963)。

50. A. Foderaro and H. L。 Garabedian, Two Group Reactor Kinetics, Nucl. Sci. Eng. 14, 22 (1962).

51. H. L. Garabedian and R. E. Lynch, Nonlinear Reactor Kinetics Analysis, Nucl. Sci. Eng. 21, 550 (1965).

52. S. Kaplan, The Property of Finality and the Analysis of Problems in Reactor Space-Time Kinetics by Various Modal Expansions, Nucl. Sci. Eng. 9, 357 (1961)。

53. John R. Lamarsh, Introduction to Nuclear Reactor Theory, AddisonWesley, Reading, Massachusetts (1966).

54. V. Luco, Space-Time Flux Synthesis Methods for the Approximate Solution of Time-Dependent Boltzmann Neutron Transport Equation, NAA-SR-11821, Atomics International, Division of North American Rockwel1 Corp。(1966). 
55. Weston M. Stacey, Jr., A Variational Multichannel Space-Time

Synthesis Method for Nonseparable Reactor Transients, Nucl. Sci. Eng. 34, 45 (1968).

56. G. C. Pomraning, A Variational Description of Dissipative Processes, J. Nucl. Energy Parts A/B 20, 617 (1966).

57. Martin Becker, Asymmetric Discontinuities in Synthesis Techniques for Initial-Value Problems, Nuc1. Sci. Eng. 34, 343 (1968).

58. Eugene L. Wachspress, Iterative Solution of EZZiptic Systems, PrenticeHall, Inc., Englewood Cliffs, New Jersey (1965).

59. R. Avery, "Theory of Coupled Reactors," Second United Nations International Conference on the Peaceful Uses of Atomic Energy, Geneva 12, 182 (1958)。

60. H. Plaza and W. H. Köhler, Coupled Reactor Kinetics Equations, Nucl. Sci. Eng. 26, 419 (1966).

61. Robert G. Cockrell and Rafael B. Perez, "Kinetic Theory of Spatial and Spectral Coupling of the Reactor Neutron Field," Neutron Dynamics and Control: Proceedings of the Symposium on Nuclear Engineering at The University of Arizona, Tucson, April 5-7, 1965, edited by David L. Hetrick and Lynn E. Weaver, Symposium Series No. 7, U.S. Atomic Energy Commission/Division of Technical Information (1966).

62. S. Kaplan, "Space-Time Kinetics," Naval Reactors Physics Handbook, Vol. 1, Selected Basic Techniques, p. 955, Naval Reactors, Division of Reactor Development, U。S. Atomic Energy Commission, Washington, D.C. (1964).

63. P. Lambropoulos and V. Luco, Functionals for Discontinuous Trial Function Flux Synthesis, submitted to Nucl。Sci. Eng.

64. D. A. Meneley, Argonne National Laboratory, personal communication (1969).

65. E. R. Cohen, "Some Topics in Reactor Kinetics," Second United Nations International Conference on the Peaceful Uses of Atomic Energy 11, 302 (1958).

66. E。 L。 Wachspress and M. Becker, Variational Multichannel Synthesis with Discontinuous Trial Functions, KAPL-3095 (1965).

67. Melville Clark, Jr。, and Kent F. Hansen, Numerical Methods of Reactor. Analysis, Academic Press, New York (1964). 